Systematic Review

\title{
The Effectiveness of Lumbar Interlaminar Epidural Injections in Managing Chronic Low Back and Lower Extremity Pain
}

\author{
Ramsin M. Benyamin, MD', Laxmaiah Manchikanti, MD², Allan T. Parr, MD, Sudhir Diwan, \\ $\mathrm{MD}^{4}$, Vijay Singh, MD , Frank J.E. Falco, $\mathrm{MD}^{6}$, Sukdeb Datta, MD7, Salahadin Abdi, MD, PhD ${ }^{8}$, \\ and Joshua A. Hirsch, MD9
}

From: ${ }^{1}$ Millennium Pain Center, Bloomington, IL, and University of Illinois, Urbana-Champaign, IL; ${ }^{2}$ Pain Management Center of Paducah,

Paducah, KY; and University of Louisville, Louisville, KY; ${ }^{3}$ Premier Pain Center, Covington, LA; ${ }^{4}$ The Spine and Pain Institute of New York, New York, NY; ${ }^{5}$ Spine Pain Diagnostics Associates, Niagara,

WI; ${ }^{6}$ Mid Atlantic Spine \& Pain

Physicians of Newark, Newark, DE; and Temple University Hospital, Philadelphia, PA; ${ }^{7}$ Laser Spine \&

Pain Institute, New York, New York; ${ }^{8}$ Beth Israel Deaconess Medical Center, Brookline, MA; and ${ }^{8}$ Harvard Medical School, Boston, MA; and ${ }^{9}$ Massachusetts General Hospital, Harvard Medical School, Boston,

Author affiliation information on pageE391

Address correspondence: Ramsin Benyamin, MD

Millennium Pain Center 1015 S. Mercer

Bloomington, IL 61701 E-mail: ramsinbenyamin@yahoo.

Disclaimer: Dr. Benyamin is a clinical investigator with Epimed and receives research support from Cephalon/Teva, BioDelivery Sciences International, Inc., Mundipharma Research GmbH \& Co., AstraZeneca, Purdue Pharma, LP, and Theravance.

Dr. Datta receives research support from Sucampo Pharmaceuticals and an honorarium from Smith and Nephew. Dr. Hirsch is a consultant for CareFusion and receives royalties

for products related to vertebral augmentation. He also participated in an Aetrium focus group and received compensation

Conflict of interest: None.

Manuscript received: 03/05/2012 Accepted for publication: 05/12/2012

Free full manuscript: www.painphysicianjournal.com
Background: Intervertebral disc herniation, spinal stenosis, intervertebral disc degeneration without disc herniation, and post lumbar surgery syndrome are the most common diagnoses of chronic persistent low back and lower extremity symptoms, resulting in significant economic, societal, and health care impact. Epidural injections are one of the most commonly performed interventions in the United States in managing chronic low back pain. However the evidence is highly variable among different techniques utilized - namely interlaminar, caudal, and transforaminal - and for various conditions, namely - intervertebral disc herniation, spinal stenosis, and discogenic pain without disc herniation or radiculitis. Multiple systematic reviews conducted in the evaluation of the effectiveness of interlaminar epidural injections have been marred with controversy. Consequently, the debate continues with regards to the effectiveness, indications, and medical necessity of interlaminar epidural injections.

Study Design: A systematic review of lumbar interlaminar epidural injections with or without steroids.

Objective: To evaluate the effect of lumbar interlaminar epidural injections with or without steroids in managing various types of chronic low back and lower extremity pain emanating as a result of disc herniation or radiculitis, spinal stenosis, and chronic discogenic pain.

Methods: The available literature on lumbar interlaminar epidural injections with or without steroids in managing various types of chronic low back pain with or without lower extremity pain was reviewed. The quality assessment and clinical relevance criteria utilized were the Cochrane Musculoskeletal Review Group criteria as utilized for interventional techniques for randomized trials and the criteria developed by the Newcastle-Ottawa Scale criteria for observational studies.

The level of evidence was classified as good, fair, or limited based on the quality of evidence developed by the U.S. Preventive Services Task Force (USPSTF).

Data sources included relevant literature identified through searches of PubMed and EMBASE from 1966 to December 2011, and manual searches of the bibliographies of known primary and review articles.

Outcome Measures: The primary outcome measure was pain relief (short-term relief = up to 6 months and long-term > 6 months). Secondary outcome measures were improvement in functional status, psychological status, return to work, and reduction in opioid intake.

Results: Overall, 82 lumbar interlaminar trials were identified. All non-randomized studies without fluoroscopy and randomized trials not meeting the inclusion criteria were excluded. Overall, 15 randomized trials and 11 non-randomized studies were included in the analysis. Analysis was derived mainly from fluoroscopically-guided randomized trials and non-randomized studies.

The evidence is good for radiculitis secondary to disc herniation with local anesthetics and steroids, fair with local anesthetic only; whereas it is fair for radiculitis secondary to spinal stenosis with local anesthetic and steroids, and fair for axial pain without disc herniation with local anesthetic with or without steroids, with fluoroscopically-guided epidural injections.

Limitations: The limitations of this study include that we were unable to perform meta- 
analysis for disc herniation, and the paucity of evidence for discogenic pain and spinal stenosis. Further, methodological criteria have been highly variable along with sample sizes. The studies were heterogenous.

Conclusion: The evidence based on this systematic review is good for lumbar epidural injections under fluoroscopy for radiculitis secondary to disc herniation with local anesthetic and steroids, fair with local anesthetic only; whereas it is fair for radiculitis secondary to spinal stenosis with local anesthetic and steroids, and fair for axial pain without disc herniation with local anesthetic with or without steroids.

Key words: Chronic low back pain, lower extremity pain, disc herniation, radiculitis, spinal stenosis, discogenic pain, lumbar interlaminar epidural injections, fluoroscopy

Pain Physician 2012; 15:E363-E404

$\mathrm{R}$ ising incidences of chronic low back pain with or without lower extremity pain and explosive increases in volume and intensity of treatment modalities for management of low back pain are major strains on the health care system (1-40). The most invasive modality, surgery, is usually performed for the most common diagnosis for low back and leg pain: disc herniation, spinal stenosis, and degenerative spondylolisthesis $(16,17,20,30,37,41-45)$. If conservative treatment fails, then surgery or epidural injections are commonly performed for chronic persistent pain of disc herniation with or without radiculitis, axial or discogenic pain without facet joint pain, spinal stenosis, and post lumbar surgery syndrome.

Epidural injections are administered by accessing the lumbar epidural space by multiple routes including interlaminar, caudal, and transforaminal $(1,12,15,20,26,28,46-60)$. While significant differences have been described between these 3 approaches, interlaminar entry is considered to deliver the medication closely to the assumed site of pathology, even though the transforaminal approach is considered the targetspecific modality requiring the smallest volume to reach the primary site of pathology $(1,26,46-59)$. Caudal epidurals are considered as the safest and easiest with minimal risk of inadvertent dural puncture, and preferred modality in post surgery syndrome, even though requiring relatively high volumes $(1,47)$.

The disadvantages also are multiple, which are described as lack of target specificity, the distribution of injectate into the dorsal space rather than ventrolateral space, lack of spread to multiple segments, excessive spread, and dural puncture. Multiple variations in the technique and the advantages and disadvantages of fluoroscopic display patterns have been described over the years (61-73). In a survey of the technical aspects of epidural steroid injections, Cluff et al (63) found only $30 \%$ of lumbar epidurals were performed under fluoroscopy in academic institutions; whereas, it was $77 \%$ in private practices. White et al (66) reported incorrect needle placement in $30.4 \%$ of patients when the lumbar interlaminar route was performed without fluoroscopy. Bartynski et al (68) described incorrect needle positioning during lumbar epidural steroid injection with loss of air pressure resistance in $25.7 \%$ of patients. Fredman et al (67) studied 50 patients with failed back surgery syndrome. The results showed that the epidural catheter did not pass through the predetermined intervertebral space in 35 cases and the contrast medium did not reach the level of pathology. Liu et al (69) prospectively evaluated with a 20-gauge Tuohy needle for lumbar epidural steroid injections and concluded that the success rate was only $92 \%$.

Botwin et al (73) evaluated lumbar interlaminar epidural injections and epidurography pattern. They showed that dorsal contrast of flow occurred in $100 \%$ of injections; however, ventral spread of the contrast was seen only in $36 \%$ of the patients. They showed a unilateral filling pattern in $84 \%$ of the patients; whereas, it was bilateral in $16 \%$. They also showed that the mean number levels of flow contrast cephalad from the injection site was 1.28 and caudally it was 0.88 with injection of a total of $5 \mathrm{~mL}$ of contrast. Weil et al (61) also evaluated fluoroscopic analysis of lumbar epidural contrast spread after lumbar interlaminar injection. They showed that the contrast spread was affected by needle placement, with other variables kept equal in performance, with the recommendation that fluoroscopy be used. They showed the spread was greater than one segment caudally more than $75 \%$ of the time under all variables. Anterior versus posterior epidural spread on the lateral view was approximately even over all cases and anterior spread was found more often when the needle was within the root of the distal spinous process tip. In another case report (62), for interlaminar epidural injections, using the loss of resistance technique and fluoroscopy without epidurogram, the rate of suboptimal injection was $12.3 \%$. Candido et al (72) 
evaluated fluoroscopically-guided lumbar epidural steroid injections and noted the epidural patterns with a lateral parasagittal approach and compared to a transforaminal approach. They reached the conclusion that the parasagittal interlaminar epidural approach was superior to the transforaminal approach.

Choi and Barbella (65) in an evaluation of contrast patterns of interlaminar epidural injections showed excellent spread of contrast into the nerve root and the ventral epidural space in all patients utilizing a paramedian approach. Whitlock et al (70) evaluated the influence of needle position on injectate spread with lumbar interlaminar injections in 460 patients. They concluded that epidural injection flow was highly variable, both among patients and between injections in a single patient. Midline injections were less likely to result in unilateral flow than a more lateral approach. Patients with previous spinal operations were more likely to have cephalad or caudad flow of less than one vertebral level than patients without a history of spinal operation. Rabinovitch et al (71) evaluated the influence of lumbar epidural injection volume on pain relief in a review of the existing literature. They showed a positive correlation between larger volumes of fluid injected into the epidural space and greater relief of radicular leg pain and/or low back pain. Identification of the epidural space with optical spectroscopy was described in a swine study with the conclusion that spectroscopic information obtained with the optical spinal needle is complementary to fluoroscopic measures, and it could potentially allow for reliable identification of the epidural space during needle placement.

Even though radicular artery injection and paralysis, etc. have been reported in multiple cases, there has been only one case report with interlaminar epidural injections (74). Much has been described about the radiculomedullary artery and its location (75). Major complications with spinal cord infarction have been described with transforaminal epidural injections in multiple reports (76-84).

Epidural procedures continue to be debated regarding their effectiveness, indications, and medical necessity $(1,12,15,20,26-29,46-50)$. The highly variable evidence ranged from indeterminate to moderate in multiple publications. The first systematic review of the effectiveness of epidural steroid injections was performed by Kepes and Duncalf in 1985 (51) which concluded that the rationale for epidural and systemic steroids had not been proven. However, in a follow-up systematic review in 1986, Benzon (59), utilizing the same studies, concluded that mechanical causes of low back pain, especially those accompanied by signs of nerve root irritation, may respond to epidural steroid injections. The differences in the conclusions of Kepes and Duncalf (51) and Benzon (59) may have been due to the fact that Kepes and Duncalf (51) included studies on systematic steroids, whereas Benzon (59) limited his analysis to studies on epidural steroid injections only.

Bogduk et al (26) concluded that the results of lumbar interlaminar epidural steroids strongly refute the utility of epidural steroids in acute sciatica. Bogduk (50) updated their recommendations in 1999, recommending against epidural steroids by the lumbar route because effective treatment required too high a number for successful treatment. In 1995, Koes et al (52) reviewed 12 trials of lumbar and caudal epidural steroid injections (combined together) and reported positive results from only 6 studies, concluding that there was no evidence for epidural steroids in managing lumbar radicular pain. Their updated review (53) with 15 trials arrived at similar conclusions that there was no evidence that epidural steroid injections are effective in patients with chronic back pain without sciatica.

Watts and Silagy (54), in a meta-analysis of the efficacy of epidural corticosteroids in the treatment of sciatica, utilized 11 studies considered of good quality, involving a total of 907 patients, and concluded that quantitative evidence from meta-analysis of pooled data from randomized trials illustrated that epidural administration of corticosteroids was effective in the management of lumbosacral radicular pain

Staal et al (15) in an updated Cochrane Review of injection therapy for subacute and chronic low back pain concluded that there was insufficient evidence to support the use of epidural injections in managing chronic low back pain. However, they concluded that it cannot be ruled out that specific subgroups of patients may respond to a specific type of injection therapy. Armon et al (56) in an assessment of the use of epidural steroid injections to treat radicular lumbosacral pain, in a poorly performed evaluation, concluded that in general, epidural steroid injections for radicular lumbosacral pain do not impact average impairment of function, need for surgery, or provide long-term pain relief beyond 3 months with a negative recommendation $(20,60)$.

Parr et al (46) reviewed the effectiveness of lumbar interlaminar epidural injections in managing chronic low back and lower extremity pain. The results showed that the available literature included only blind epi- 
dural injections without fluoroscopy. Consequently, the evidence was determined as poor.

The American Pain Society (APS) guidelines by Chou and Huffman also showed negative results for lumbar interlaminar epidural injections except for radicular pain on a short-term basis $(20,28)$. Rho and Tang $(85)$ in describing the efficacy of lumbar epidural steroid injections, which also included all 3 approaches, showed strong evidence for transforaminal epidural steroid injections, but the evidence showed only short-term efficacy of interlaminar epidural steroid injections and caudal epidural injections in the management of low back and radicular pain. They concluded that lumbar epidural steroids can be an effective tool in the conservative management of low back pain with radicular symptoms. Multiple evaluators in the past have reached favorable conclusions with moderate effectiveness in managing lumbar radiculopathy, when these were separated from blind interlaminar epidural injections. There is also emerging evidence with studies under fluoroscopy.

Due to the ongoing debate and lack of significant evidence, this systematic review is undertaken to evaluate the effects of lumbar interlaminar epidural injections in managing chronic low back and lower extremity pain secondary to lumbar disc herniation and radiculitis, spinal stenosis, and chronic low back pain of discogenic origin without radiculitis or disc herniation.

\subsection{Methods}

The methodology utilized in this systematic review followed the review process derived from evidencebased systematic reviews and meta-analysis of randomized trials and observational studies $(1,15,86-96)$, Consolidated Standards of Reporting Trials (CONSORT) guidelines for the conduct of randomized trials (97100), Standards for Reporting Observational Studies (STROBE) (101), Cochrane guidelines $(15,91,92)$, Chou and Huffman's guidelines (20), and quality of reporting of analysis (88).

\subsection{Criteria for Considering Studies for This Review}

\subsubsection{Types of Studies}

Randomized controlled trials

Non-randomized observational studies

Case reports and reviews for adverse effects

\subsubsection{Types of Participants}

Participants of interest were adults aged at least 18 years with chronic low back and lower extremity pain of at least 3 months duration.

Participants must have failed previous pharmacotherapy, exercise therapy, etc., prior to starting interventional pain management techniques.

\subsubsection{Types of Interventions}

The interventions were lumbar interlaminar epidural injections for chronic low back and/or lower extremity pain. All randomized trials with proper inclusion criteria and appropriately performed non-randomized studies with proper technique under fluoroscopic or CT guidance.

\subsubsection{Types of Outcome Measures}

- The primary outcome parameter was pain relief.

- The secondary outcome measures were functional improvement; change in psychological status; return to work; reduction or elimination of opioid use, other drugs, or other interventions; and complications.

- At least 2 of the review authors independently, in an unblinded standardized manner, assessed the outcomes measures. Any disagreements between reviewers were resolved by a third author and consensus.

\subsection{Literature Search}

Searches were performed from the following sources without language restrictions:

1. PubMed from 1966 www.ncbi.nlm.nih.gov/sites/entrez?db=pubmed

2. EMBASE from 1980 www.embase.com

3. Cochrane Library www.thecochranelibrary.com/view/0/index.html

4. U.S. National Guideline Clearinghouse (NGC) www.guideline.gov

5. Previous systematic reviews and cross references

6. Clinical Trials

www.clinicaltrials.gov

The search period was from 1966 through December 2011.

\subsection{Search Strategy}

The search strategy emphasized chronic low back and lower extremity pain, disc herniation, discogenic pain, spinal stenosis, and radiculitis treated with lumbar interlaminar epidural injections. 
At least 2 of the review authors independently, in an unblinded standardized manner, performed each search. Accuracy was confirmed by a statistician. All searches were combined to obtain a unified search strategy. Any disagreements between reviewers were resolved by a third author and consensus.

\subsection{Data Collection and Analysis}

The review focused on randomized trials, fluoroscopically-guided observational studies, and reports of complications. The population of interest was patients suffering with chronic low back and lower extremity pain for at least 3 months. Only lumbar interlaminar epidural injections with or without steroids were evaluated. All of the studies providing appropriate management and with outcome evaluations of one month or longer and statistical evaluations were reviewed. Reports without appropriate diagnosis, non-systematic reviews, book chapters, and case reports will be excluded.

\subsubsection{Selection of Studies}

- In an unblinded, standardized manner, 2 review authors screened the abstracts of all identified studies against the inclusion criteria.

- All articles with possible relevance were then retrieved in full text for comprehensive assessment of internal validity, quality, and adherence to inclusion criteria.

\subsubsection{Inclusion and Exclusion Criteria}

The following are the inclusion and exclusion criteria:

1. Are the patients described in sufficient detail to al- low one to decide whether they are comparable to those who are treated in interventional pain management clinical practices?

A. Setting - office, hospital, outpatient, inpatient

B. Physician - interventional pain physician, general physician, anesthesiologist, physiatrist, neurologist, rheumatologist, orthopedic surgeon, neurosurgeon, etc.

C. Patient characteristics - duration of pain

D. Non-interventional techniques or surgical intervention in the past

2. Is the intervention described in sufficient detail to enable one to apply its use to patients in interventional pain management settings?
A. Nature of intervention
B. Frequency of intervention
C. Duration of intervention

3. Were clinically relevant outcomes measured?
A. Proportion of pain relief
B. Disorder/specific disability
C. Functional improvement
D. Allocation of eligible and non-eligible patients to return to work
E. Ability to work

\subsubsection{Clinical Relevance}

The clinical relevance of the included studies were evaluated according to 5 questions recommended by the Cochrane Back Review Group (Table 1) $(90,102)$. Each question was scored as positive (+) if the clinical relevance item was met, negative (-) if the item was not met, and unclear (?) if data were not available to answer the question.

Table 1. Clinical relevance questions.

\begin{tabular}{|c|c|c|}
\hline & $\begin{array}{l}\mathbf{N} \\
(-)\end{array}$ & $\begin{array}{c}\mathrm{U} \\
\text { (unclear) }\end{array}$ \\
\hline \multicolumn{3}{|c|}{$\begin{array}{l}\text { A) Are the patients described in detail so that one can decide whether they are comparable to those who are } \\
\text { treated in a clinical practice? }\end{array}$} \\
\hline \multicolumn{3}{|c|}{$\begin{array}{l}\text { B) Are the interventions and treatment settings described in sufficient detail to apply its use in clinical } \\
\text { practice? }\end{array}$} \\
\hline \multicolumn{3}{|l|}{ C) Were clinically relevant outcomes measured and reported? } \\
\hline \multicolumn{3}{|l|}{ D) Is the size of the effect clinically meaningful? } \\
\hline E) Do the likely treatment benefits outweigh the potential harms? & & \\
\hline
\end{tabular}

Scoring adapted and modified from Staal JB, et al. Injection therapy for subacute and chronic low-back pain. Cochrane Database Syst Rev 2008; 3:CD001824 (102). 


\subsubsection{Methodological Quality or Validity Assessment}

Even though none of these instruments or criteria have been systematically assessed, the advantages and disadvantages of each system were debated.

The methodological quality assessment was performed by 2 review authors who independently assessed, in an unblinded standardized manner, the internal validity of all the studies.

Any discrepancies or conflicts were arbitrated by a third reviewer to either reach a consensus agreement or break a tie. If there was a conflict of interest with the reviewed manuscripts with authorship or any other type of conflict, the involved authors did not review the manuscripts for quality assessment or evidence synthesis.

The quality of each individual article used in this analysis was assessed by Cochrane review criteria (Table 2) (91) for randomized trials and Newcastle-Ottawa Scale for observational studies (Tables 3 and 4) $(103,104)$. For nonrandomized observational studies, the patient population should have had at least 50 total or at least 25 in each group if they were comparison groups.

Authors with a perceived conflict of interest for any manuscript were recused from reviewing the manuscript.

For adverse effects, confounding factors, etc., it was not possible to use quality assessment criteria. Thus, these were considered based on interpretation of the reports published and critical analysis of the literature.

Only the randomized trials meeting the inclusion criteria with at least $50 \%$ of the criteria were utilized for analysis. However, studies scoring lower were described and provided with an opinion and critical analysis.

Observational studies had to meet a minimum of $50 \%$ of the criteria for cohort and case-control studies. Studies scoring less were also described and provided with an opinion and a critical analysis.

If the literature search provided at least 5 randomized trials meeting the inclusion criteria and they were homogenous for each modality and condition evaluated, a meta-analysis was performed.

All lumbar interlaminar epidural injections were also evaluated separately for disc herniation, discogenic pain, and spinal stenosis.

\subsubsection{Data Extraction and Management}

Two review authors independently, in an unblinded standardized manner, extracted the data from the included studies. Disagreements were resolved by discussion between the 2 reviewers; if no consensus could be reached, a third author was called in to break the impasse.

\subsubsection{Assessment of Heterogeneity}

Whenever meta-analyses were conducted, the Isquared (I2) statistic was used to identify heterogeneity (104). Combined results with $12>50 \%$ were considered substantially heterogenous.

Analysis of the evidence was based on the condition (i.e., disc herniation or spinal stenosis) to reduce any clinical heterogeneity.

\subsubsection{Measurement of Treatment Effect in Data Synthesis (Meta-Analysis)}

Data was summarized using meta-analysis when at least 5 studies per type of disorder were available that met the inclusion criteria (e.g., lumbar disc herniation or spinal stenosis, etc.).

Qualitative (the direction of a treatment effect) and quantitative (the magnitude of a treatment effect) conclusions were evaluated. Random-effects metaanalysis to pool data was also used (105).

The minimum amount of change in pain score to be clinically meaningful has been described as a 2-point change on a scale of 0 to 10 (or 20 percentage points), based on findings in trials studying general chronic pain (106), chronic musculoskeletal pain (107), and chronic low back pain $(86-88,90,107-109)$, which have been commonly utilized. However, recent descriptions of clinically meaningful improvement showed either pain relief or functional status as $50 \%$ (110-120). Consequently, for this analysis, we utilize clinically meaningful pain relief of at least a 3-point change on an 11 -point scale of 0 to 10 , or $50 \%$ pain relief from the baseline, as clinically significant, and functional status improvement of $40 \%$ or more.

\subsubsection{Integration of Heterogeneity}

The evidence was assessed separately by administration to each condition. A meta-analysis was performed only if there were at least 5 studies meeting inclusion criteria for each variable.

Statistical heterogeneity was explored using univariate meta-regression (121).

\subsubsection{Software Used for Measurement}

The data was analyzed using SPSS Version 9.0.1 statistical software (SPSS Inc., Chicago, IL), Microsoft Ac- 
Table 2. Randomized controlled trials quality rating system.

\begin{tabular}{|c|c|c|c|}
\hline A & $\begin{array}{l}\text { 1. Was the method of } \\
\text { randomization adequate? }\end{array}$ & $\begin{array}{l}\text { A random (unpredictable) assignment sequence. Examples of adequate methods are coin toss (for studies } \\
\text { with } 2 \text { groups), rolling a die (for studies with } 2 \text { or more groups), drawing of balls of different colors, drawing } \\
\text { of ballots with the study group labels from a dark bag, computer-generated random sequence, pre-ordered } \\
\text { sealed envelopes, sequentially ordered vials, telephone call to a central office, and pre-ordered list of treatment } \\
\text { assignments. Examples of inadequate methods are alternation, birth date, social insurance/security number, } \\
\text { date in which they are invited to participate in the study, and hospital registration number. }\end{array}$ & $\begin{array}{l}\text { Yes/No/ } \\
\text { Unsure }\end{array}$ \\
\hline B & $\begin{array}{l}\text { 2. Was the treatment } \\
\text { allocation concealed? }\end{array}$ & $\begin{array}{l}\text { Assignment generated by an independent person not responsible for determining the eligibility } \\
\text { of the patients. This person has no information about the persons included in the trial and has no } \\
\text { influence on the assignment sequence or on the decision about eligibility of the patient. }\end{array}$ & $\begin{array}{l}\text { Yes/No/ } \\
\text { Unsure }\end{array}$ \\
\hline \multirow[t]{4}{*}{ C } & \multicolumn{2}{|c|}{ Was knowledge of the allocated interventions adequately prevented during the study? } & \\
\hline & $\begin{array}{l}\text { 3. Was the patient blinded to } \\
\text { the intervention? }\end{array}$ & $\begin{array}{l}\text { This item should be scored "yes" if the index and control groups are indistinguishable for the } \\
\text { patients or if the success of blinding was tested among the patients and it was successful. }\end{array}$ & $\begin{array}{l}\text { Yes/No/ } \\
\text { Unsure }\end{array}$ \\
\hline & $\begin{array}{l}\text { 4. Was the care provider } \\
\text { blinded to the intervention? }\end{array}$ & $\begin{array}{l}\text { This item should be scored "yes" if the index and control groups are indistinguishable for the care } \\
\text { providers or if the success of blinding was tested among the care providers and it was successful. }\end{array}$ & $\begin{array}{l}\text { Yes/No/ } \\
\text { Unsure }\end{array}$ \\
\hline & $\begin{array}{l}\text { 5. Was the outcome assessor } \\
\text { blinded to the intervention? }\end{array}$ & $\begin{array}{l}\text { Adequacy of blinding should be assessed for the primary outcomes. This item should be scored "yes" } \\
\text { if the success of blinding was tested among the outcome assessors and it was successful or: } \\
\text {-for patient-reported outcomes in which the patient is the outcome assessor (e.g., pain, disability): } \\
\text { the blinding procedure is adequate for outcome assessors if participant blinding is scored "yes" } \\
\text {-for outcome criteria assessed during scheduled visit and that supposes a contact between } \\
\text { participants and outcome assessors (e.g., clinical examination): the blinding procedure is adequate if } \\
\text { patients are blinded, and the treatment or adverse effects of the treatment cannot be noticed during } \\
\text { clinical examination } \\
\text {-for outcome criteria that do not suppose a contact with participants (e.g., radiography, magnetic } \\
\text { resonance imaging): the blinding procedure is adequate if the treatment or adverse effects of the } \\
\text { treatment cannot be noticed when assessing the main outcome } \\
\text {-for outcome criteria that are clinical or therapeutic events that will be determined by the } \\
\text { interaction between patients and care providers (e.g., co-interventions, hospitalization length, } \\
\text { treatment failure), in which the care provider is the outcome assessor: the blinding procedure is } \\
\text { adequate for outcome assessors if item "4" (caregivers) is scored "yes" } \\
\text {-for outcome criteria that are assessed from data of the medical forms: the blinding procedure is } \\
\text { adequate if the treatment or adverse effects of the treatment cannot be noticed on the extracted data. }\end{array}$ & $\begin{array}{l}\text { Yes/No/ } \\
\text { Unsure }\end{array}$ \\
\hline \multirow[t]{3}{*}{$\mathrm{D}$} & \multicolumn{2}{|c|}{ Were incomplete outcome data adequately addressed? } & \\
\hline & $\begin{array}{l}\text { 6. Was the drop-out rate } \\
\text { described and acceptable? }\end{array}$ & $\begin{array}{l}\text { The number of participants who were included in the study but did not complete the } \\
\text { observation period or were not included in the analysis must be described and reasons given. If } \\
\text { the percentage of withdrawals and drop-outs does not exceed } 20 \% \text { for short-term follow-up and } \\
30 \% \text { for long-term follow-up and does not lead to substantial bias a "yes" is scored. (N.B. these } \\
\text { percentages are arbitrary, not supported by literature). }\end{array}$ & $\begin{array}{l}\text { Yes/No/ } \\
\text { Unsure }\end{array}$ \\
\hline & $\begin{array}{l}\text { 7. Were all randomized } \\
\text { participants analyzed in the group } \\
\text { to which they were allocated? }\end{array}$ & $\begin{array}{l}\text { All randomized patients are reported/analyzed in the group they were allocated to by } \\
\text { randomization for the most important moments of effect measurement (minus missing values) } \\
\text { irrespective of non-compliance and co-interventions. }\end{array}$ & $\begin{array}{l}\text { Yes/No/ } \\
\text { Unsure }\end{array}$ \\
\hline $\mathrm{E}$ & $\begin{array}{l}\text { 8. Are reports of the study } \\
\text { free of suggestion of selective } \\
\text { outcome reporting? }\end{array}$ & $\begin{array}{l}\text { In order to receive a "yes," the review author determines if all the results from all pre-specified } \\
\text { outcomes have been adequately reported in the published report of the trial. This information } \\
\text { is either obtained by comparing the protocol and the report, or in the absence of the protocol, } \\
\text { assessing that the published report includes enough information to make this judgment. }\end{array}$ & $\begin{array}{l}\text { Yes/No/ } \\
\text { Unsure }\end{array}$ \\
\hline \multirow[t]{5}{*}{$\mathrm{F}$} & \multicolumn{2}{|l|}{ Other sources of potential bias: } & \\
\hline & $\begin{array}{l}\text { 9. Were the groups similar at } \\
\text { baseline regarding the most } \\
\text { important prognostic indicators? }\end{array}$ & $\begin{array}{l}\text { In order to receive a "yes," groups have to be similar at baseline regarding demographic factors, } \\
\text { duration and severity of complaints, percentage of patients with neurological symptoms, and } \\
\text { value of main outcome measure(s). }\end{array}$ & $\begin{array}{l}\text { Yes/No/ } \\
\text { Unsure }\end{array}$ \\
\hline & $\begin{array}{l}\text { 10. Were co-interventions } \\
\text { avoided or similar? }\end{array}$ & $\begin{array}{l}\text { This item should be scored "yes" if there were no co-interventions or they were similar between } \\
\text { the index and control groups. }\end{array}$ & $\begin{array}{l}\text { Yes/No/ } \\
\text { Unsure }\end{array}$ \\
\hline & $\begin{array}{l}\text { 11. Was the compliance } \\
\text { acceptable in all groups? }\end{array}$ & $\begin{array}{l}\text { The reviewer determines if the compliance with the interventions is acceptable, based on the } \\
\text { reported intensity, duration, number and frequency of sessions for both the index intervention } \\
\text { and control intervention(s). For example, physiotherapy treatment is usually administered over } \\
\text { several sessions; therefore, it is necessary to assess how many sessions each patient attended. For } \\
\text { single-session interventions (e.g., surgery), this item is irrelevant. }\end{array}$ & $\begin{array}{l}\text { Yes/No/ } \\
\text { Unsure }\end{array}$ \\
\hline & $\begin{array}{l}\text { 12. Was the timing of the outcome } \\
\text { assessment similar in all groups? }\end{array}$ & $\begin{array}{l}\text { Timing of outcome assessment should be identical for all intervention groups and for all } \\
\text { important outcome assessments. }\end{array}$ & $\begin{array}{l}\text { Yes/No/ } \\
\text { Unsure }\end{array}$ \\
\hline
\end{tabular}

Adapted and modified from Furlan AD, et al; Editorial Board, Cochrane Back Review Group. 2009 updated method guidelines for systematic reviews in the Cochrane Back Review Group. Spine (Phila Pa 1976) 2009; 34:1929-1941 (91). 
Table 3. Newcastle-Ottawa quality assessment scale for case control studies.

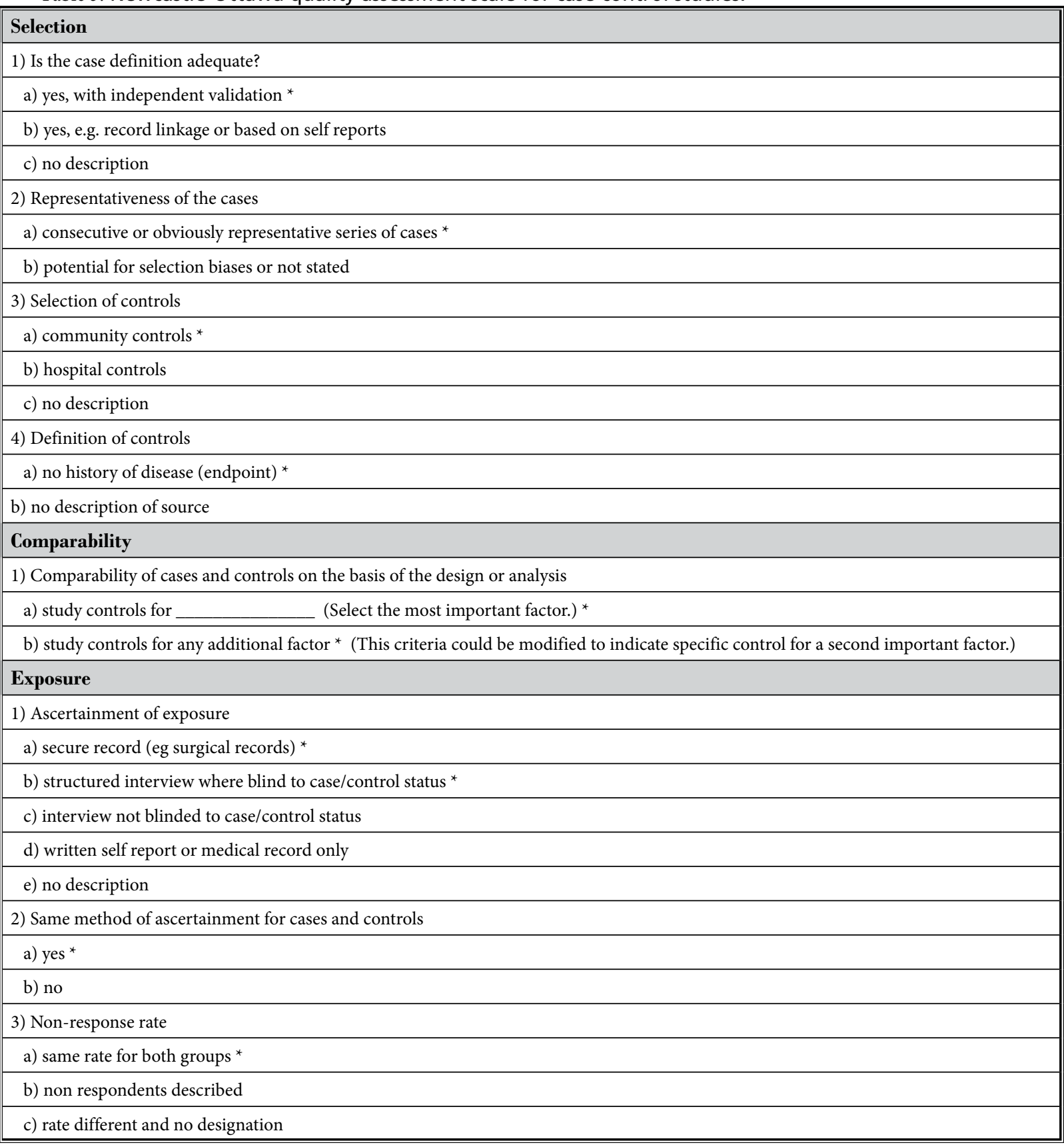

Note: A study can be awarded a maximum of one star for each numbered item within the Selection and Exposure categories. A maximum of two stars can be given for Comparability.

Wells GA, et al. The Newcastle-Ottawa Scale (NOS) for assessing the quality of nonrandomized studies in meta-analysis. www.ohri.ca/programs/clinical_epidemiology/oxford.asp (103). 


\section{Effectiveness of Lumbar Interlaminar Epidural Injections}

Table 4. Newcastle-Ottawa quality assessment scale for cohort studies.

\begin{tabular}{|c|}
\hline Selection \\
\hline 1) Representativeness of the exposed cohort \\
\hline a) truly representative of the average ___ (describe) in the community * \\
\hline b) somewhat representative of the average ___ in the community * \\
\hline c) selected group of users, e.g. nurses, volunteers \\
\hline d) no description of the derivation of the cohort \\
\hline 2) Selection of the non exposed cohort \\
\hline a) drawn from the same community as the exposed cohort * \\
\hline b) drawn from a different source \\
\hline c) no description of the derivation of the non exposed cohort \\
\hline 3) Ascertainment of exposure \\
\hline a) secure record (e.g. surgical records) * \\
\hline b) structured interview ${ }^{*}$ \\
\hline c) written self report \\
\hline d) no description \\
\hline 4) Demonstration that outcome of interest was not present at start of study \\
\hline a) yes* \\
\hline b) no \\
\hline Comparability \\
\hline 1) Comparability of cohorts on the basis of the design or analysis \\
\hline a) study controls for ___ (select the most important factor) ${ }^{*}$ \\
\hline b) study controls for any additional factor ${ }^{\star}$ (This criteria could be modified to indicate specific control for a second important factor.) \\
\hline Outcome \\
\hline 1) Assessment of outcome \\
\hline a) independent blind assessment * \\
\hline b) record linkage* \\
\hline c) self report \\
\hline d) no description \\
\hline 2) Was follow-up long enough for outcomes to occur \\
\hline a) yes (select an adequate follow-up period for outcome of interest)* \\
\hline b) no \\
\hline 3) Adequacy of follow-up of cohorts \\
\hline a) complete follow-up - all subjects accounted for * \\
\hline $\begin{array}{l}\text { b) subjects lost to follow-up unlikely to introduce bias - small number lost - > __ } \% \text { (select an adequate \%) follow-up, or description } \\
\text { provided of those lost)* }\end{array}$ \\
\hline c) follow-up rate $<\ldots \ldots$ (select an adequate $\%$ ) and no description of those lost \\
\hline d) no statement \\
\hline
\end{tabular}

Note: A study can be awarded a maximum of one star for each numbered item within the Selection and Outcome categories. A maximum of two stars can be given for Comparability.

Wells GA, et al. The Newcastle-Ottawa Scale (NOS) for assessing the quality of nonrandomized studies in meta-analysis. www.ohri.ca/programs/clinical_epidemiology/oxford.asp (103). 
cess 2003, and Microsoft Excel 2003 (Microsoft Corporation, Redmond, WA) (122).

Meta-analyses were performed with Comprehensive Meta-Analysis Software Version 2.0 for Windows (Biostat Inc., Englewood, NJ) (123).

\subsection{Summary Measures}

Summary measures included $50 \%$ or more reduction of pain in at least $40 \%$ of the patients, or at least a 3 point decrease in pain scores and a relative risk of adverse events including side effects.

\subsection{Analysis of Evidence}

The analysis of the evidence was performed based on United States Preventive Services Task Force (USPSTF) criteria as illustrated in Table 5, criteria which has been utilized by multiple authors $(19,20,22,95,96,124)$.

The analysis was conducted using 3 levels of evidence ranging from good, fair, or limited.

At least 2 of the review authors independently, in an unblinded standardized manner, analyzed the evidence. Any disagreements between reviewers were resolved by a third author and consensus. If there were any conflicts of interest (e.g., authorship), those reviewers were recused from assessment and analysis.

\subsection{Outcome of the Studies}

In the randomized trials, a study was judged to be positive if the lumbar interlaminar epidural injection therapy was clinically relevant and effective, either with a placebo control or active control. This indicates that the difference in effect for the primary outcome measure is statistically significant on the conventional $5 \%$ level. In a negative study, no difference between the study treatments or no improvement from baseline is identified. Further, the outcomes were judged at the reference point with positive or negative results reported at one month, 3 months, 6 months, and one year.

For observational studies, a study was judged to be positive if the epidural injection therapy was effective, with outcomes reported at the reference point with positive or negative results at one month, 3 months, 6 months, and one year. However, observational studies were only included in the evidence synthesis if there was less than 5 randomized trials meeting inclusion criteria for evidence synthesis for each condition (i.e., disc herniation, spinal stenosis, and discogenic pain).

\subsection{Results}

Figure 1 shows a flow diagram of study selection as recommended by Preferred Reporting Items for Systematic Reviews and Meta-Analyses (PRISMA) (89). There were 82 studies considered for inclusion $(72,110,111,117,118,125-199)$.

Of the 82 lumbar epidural trials iden-
tified, 54 were excluded $(117,118,125-$ $127,131,132,134,137,138,143,146,148-150,152,154-$ $164,166-173,175,176-178,183-193,195-197)$. Table 6 shows the reasons for exclusion for lumbar interlaminar randomized trials and fluoroscopically-guided observational studies. Of these, only 15 were randomized trials (125$127,131,132,134,137,143,148,150,162,167,170,184,197)$ and 11 were non-randomized studies $(138,149,163,164$ $, 169,172,173,175,178,183,193)$. The remaining non-randomized studies were performed without fluoroscopy $(146,152,154-161,171,185-187)$.

Table 7 illustrates the characteristics of randomized trials without fluoroscopy considered for in-

Table 5. Method for grading the overall strength of the evidence for an intervention.

\begin{tabular}{|l|l||}
\hline Grade & Definition \\
\hline Good & $\begin{array}{l}\text { Evidence includes consistent results from well-designed, well-conducted studies in representative populations that directly } \\
\text { assess effects on health outcomes (at least 2 consistent, higher-quality RCTs or studies of diagnostic test accuracy). }\end{array}$ \\
\hline Fair & $\begin{array}{l}\text { Evidence is sufficient to determine effects on health outcomes, but the strength of the evidence is limited by the number, } \\
\text { quality, size, or consistency of included studies; generalizability to routine practice; or indirect nature of the evidence on health } \\
\text { outcomes (at least one higher-quality trial or study of diagnostic test accuracy of sufficient sample size; } 2 \text { or more higher-quality } \\
\text { trials or studies of diagnostic test accuracy with some inconsistency; at least } 2 \text { consistent, lower-quality trials or studies of } \\
\text { diagnostic test accuracy, or multiple consistent observational studies with no significant methodological flaws). }\end{array}$ \\
\hline $\begin{array}{l}\text { Limited or } \\
\text { poor }\end{array}$ & $\begin{array}{l}\text { Evidence is insufficient to assess effects on health outcomes because of limited number or power of studies, large and } \\
\text { unexplained inconsistency between higher-quality trials, important flaws in trial design or conduct, gaps in the chain of } \\
\text { evidence, or lack of information on important health outcomes. }\end{array}$ \\
\hline
\end{tabular}

Adapted and modified from methods developed by U.S. Preventive Services Task Force $(19,20,124)$. 


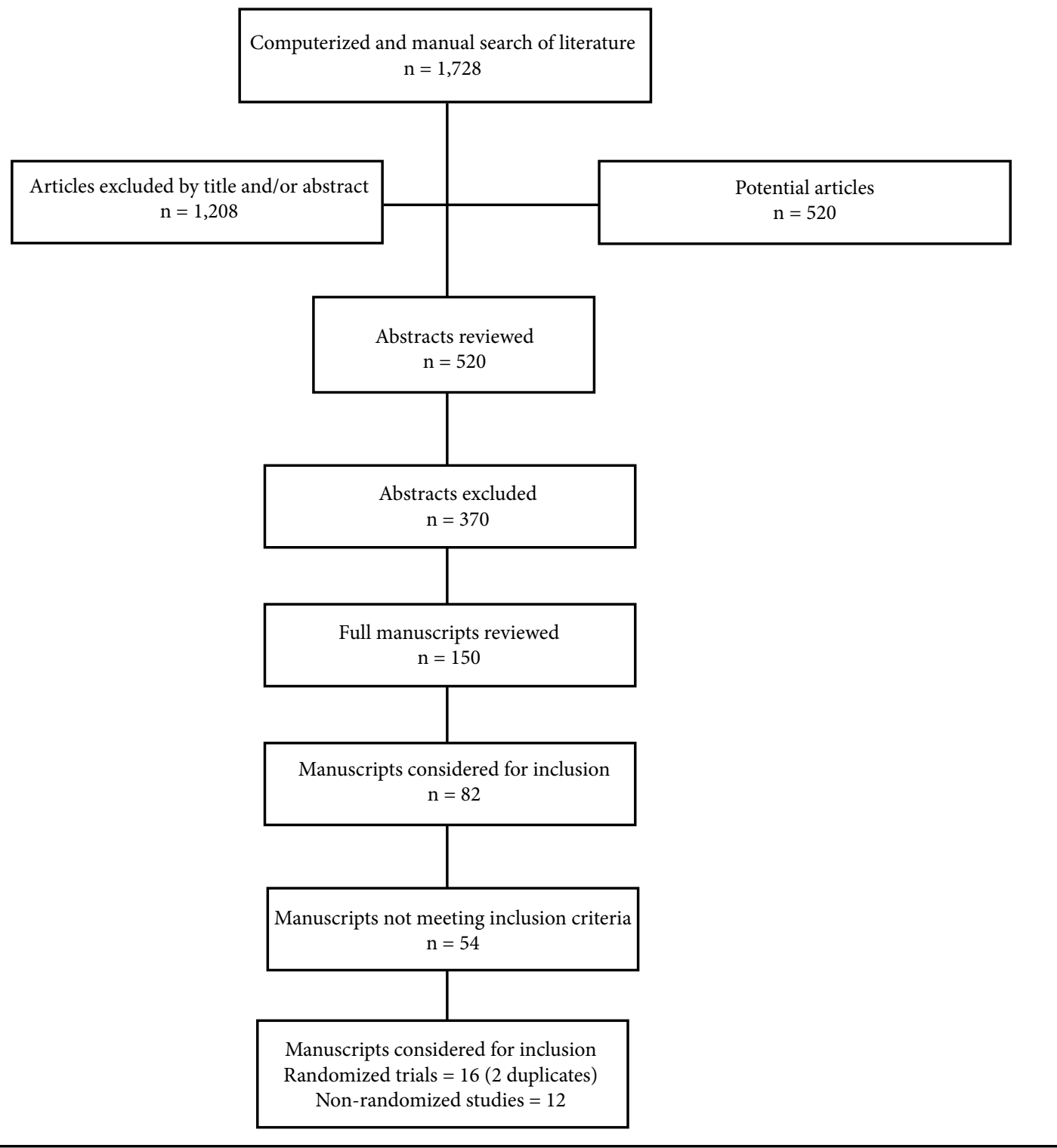

Fig. 1. The flow diagram illustrating published literature evaluating lumbar interlaminar epidural injections.

clusion. There were 12 randomized trials without fluoroscopy $\quad(128-130,133,135,136,139-142,144,153)$. Table 8 illustrates characteristics of 16 randomized trials and non-randomized studies performed under fluoroscopy $(72,110,111,145,147,151,165,174,177,179$ $182,194,198,199) \quad$ with 2 duplicate studies $(110,111,198,199)$.

\subsection{Clinical Relevance}

Of the 26 studies assessed for clinical relevance, 23 randomized studies met criteria with score of 3 of 5 or greater (Table 9).

\subsection{Methodological Quality Assessment}

A methodological quality assessment of the randomized controlled trials meeting inclusion criteria was 
Pain Physician: July/August 2012; 15:E363-E404

Table 6. List of excluded randomized trials and fluoroscopic non-randomized studies

\begin{tabular}{|c|c|c|c|c|}
\hline \multirow{2}{*}{$\begin{array}{l}\text { Manuscript } \\
\text { Author(s) }\end{array}$} & \multirow{2}{*}{$\begin{array}{l}\text { Condition } \\
\text { Studied }\end{array}$} & \multirow{2}{*}{$\begin{array}{l}\text { Number of } \\
\text { Patients }\end{array}$} & \multicolumn{2}{|c|}{ Reason for Exclusion } \\
\hline & & & $\begin{array}{l}\text { Follow- } \\
\text { up Period }\end{array}$ & Other Reason(s) \\
\hline \multicolumn{5}{|l|}{ RANDOMIZED } \\
\hline Serrao et al (125) & $\begin{array}{l}\text { Chronic low back } \\
\text { pain }\end{array}$ & 28 & 2 months & $\begin{array}{l}\text { Intrathecal midazolam compared with epidural steroid in a pilot } \\
\text { study. }\end{array}$ \\
\hline $\begin{array}{l}\text { Rodriguez Hernandez } \\
\text { et al (126) }\end{array}$ & $\begin{array}{l}\text { Diabetic } \\
\text { neuropathy }\end{array}$ & 20 & 30 days & Study of diabetic neuropathy with epidural steroid injections. \\
\hline Kikuchi et al (127) & $\begin{array}{l}\text { Postherpetic } \\
\text { neuralgia }\end{array}$ & 25 & 24 weeks & $\begin{array}{l}\text { Comparison of intrathecal versus epidural methylprednisolone in } \\
\text { intractable postherpetic neuralgia in a small number of patients. }\end{array}$ \\
\hline Klenerman et al (131) & Sciatica & 74 & NA & $\begin{array}{l}\text { The inclusion criteria was unilateral sciatica for less than } 6 \text { months, } \\
\text { thus including acute and subacute patients. }\end{array}$ \\
\hline Rocco et al (132) & $\begin{array}{l}\text { Postlaminectomy } \\
\text { syndrome }\end{array}$ & 24 & 30 days & $\begin{array}{l}\text { The effect of epidural steroids was compared with morphine in the } \\
\text { treatment of postlaminectomy syndrome in only } 24 \text { patients. }\end{array}$ \\
\hline Valat et al (134) & $\begin{array}{l}\text { Lumbar } \\
\text { radiculitis }\end{array}$ & 85 & 35 days & $\begin{array}{l}\text { The inclusion criteria was of sciatica of more than } 15 \text { and less than } \\
180 \text { days, thus including subacute and acute patients with sciatica. }\end{array}$ \\
\hline Bronfort et al (137) & $\begin{array}{l}\text { Lumbar } \\
\text { radiculitis }\end{array}$ & 32 & 52 weeks & $\begin{array}{l}\text { The study included acute and subacute pain in patients in a small } \\
\text { sample. }\end{array}$ \\
\hline Snoek et al (143) & $\begin{array}{l}\text { Lumbar disc } \\
\text { herniation }\end{array}$ & 51 & 14 months & $\begin{array}{l}\text { Authors evaluated a single epidural injection in acute and subacute } \\
\text { radiculitis. The inclusion criteria was with patients with lumbar } \\
\text { root compression syndrome of } 12 \text { days to } 36 \text { weeks duration, thus } \\
\text { including a number of acute and subacute pain patients. }\end{array}$ \\
\hline $\begin{array}{l}\text { Jirarattanaphochai et } \\
\text { al (148) }\end{array}$ & $\begin{array}{l}\text { Lumbar disc } \\
\text { herniation }\end{array}$ & 103 & 2 days & $\begin{array}{l}\text { Authors evaluated peridural methylprednisolone and wound } \\
\text { infiltration with bupivacaine for post-operative pain control after } \\
\text { posterior lumbar spine surgery. }\end{array}$ \\
\hline Price et al (150) & $\begin{array}{l}\text { Chronic low back } \\
\text { pain }\end{array}$ & 200 & Immediate & Comparison of accuracy of needle placement. \\
\hline Rasmussen et al (162) & Disc herniation & 200 & One year & $\begin{array}{l}\text { Authors evaluated epidural steroid following discectomy } \\
\text { for herniated lumbar disc and concluded that epidural } \\
\text { methylprednisolone enhances recovery after discectomy for } \\
\text { herniated disc disease without side effects. }\end{array}$ \\
\hline Lima et al (167) & $\begin{array}{l}\text { Experimental } \\
\text { trial in dogs }\end{array}$ & 14 dogs & 21 days & $\begin{array}{l}\text { An animal basic science trial to evaluate clinical and histological effects } \\
\text { of the intrathecal administration of methylprednisolone in dogs. }\end{array}$ \\
\hline Debi et al (170) & Disc herniation & 70 & One year & $\begin{array}{l}\text { Authors evaluated local application of steroids following lumbar } \\
\text { discectomy. }\end{array}$ \\
\hline Gelalis et al (184) & $\begin{array}{l}\text { Lumbar disc } \\
\text { herniation }\end{array}$ & 40 & 2 months & $\begin{array}{l}\text { Lumbar radiculitis secondary to acute and subacute pain was } \\
\text { evaluated. }\end{array}$ \\
\hline Mobaleghi et al (197) & $\begin{array}{l}\text { Disc herniation } \\
\text { and stenosis }\end{array}$ & $\begin{array}{l}\text { 60. Disc } \\
\text { herniation = } \\
\text { 32. Stenosis } \\
=28\end{array}$ & 6 months & Blind prospective evaluation. \\
\hline \multicolumn{5}{|l|}{ NON-RANDOMIZED } \\
\hline Briggs et al (138) & Spinal stenosis & 62 & 2 years & Lumbar interlaminar - appropriate data not available. \\
\hline Schaufele et al (149) & $\begin{array}{l}\text { Lumbar disc } \\
\text { herniation }\end{array}$ & 20 & One year & $\begin{array}{l}\text { A small number of patients with comparison of interlaminar versus } \\
\text { transforaminal epidural injections in a case-control report. }\end{array}$ \\
\hline Mitra et al (163) & Spinal stenosis & One & $\mathrm{NA}$ & $\begin{array}{l}\text { A single case report of overactive bladder associated with severe } \\
\text { central canal stenosis was studied. }\end{array}$ \\
\hline Stretanski (164) & $\begin{array}{l}\text { Lumbar } \\
\text { radiculitis }\end{array}$ & 10 & One week & $\begin{array}{l}\text { In this study, } \mathrm{H} \text {-reflex latency and nerve root tension sign } \\
\text { correlation was evaluated in } 10 \text { patients in a prospective } \\
\text { observational report. }\end{array}$ \\
\hline
\end{tabular}


Effectiveness of Lumbar Interlaminar Epidural Injections

Table 6 (cont.). List of excluded randomized trials and non-randomized studies performed under fluoroscopy.

\begin{tabular}{|c|c|c|c|c|}
\hline \multirow{2}{*}{$\begin{array}{l}\text { Manuscript } \\
\text { Author(s) }\end{array}$} & \multirow{2}{*}{$\begin{array}{l}\text { Condition } \\
\text { Studied }\end{array}$} & \multirow{2}{*}{$\begin{array}{l}\text { Number of } \\
\text { Patients }\end{array}$} & \multicolumn{2}{|c|}{ Reason for Exclusion } \\
\hline & & & $\begin{array}{l}\text { Follow- } \\
\text { up Period }\end{array}$ & Other Reason(s) \\
\hline Price et al (169) & Sciatica & 228 & 52 weeks & $\begin{array}{l}\text { This is a publication of another publication evaluating the cost- } \\
\text { effectiveness and safety. }\end{array}$ \\
\hline Furman et al (172) & $\begin{array}{l}\text { Lumbar } \\
\text { radiculitis }\end{array}$ & 21 & 6 weeks & A small number of patients in a pilot study. \\
\hline Smith et al (173) & $\begin{array}{l}\text { Symptomatic } \\
\text { lumbar spinal } \\
\text { stenosis }\end{array}$ & 38 & 6 weeks & A small retrospective analysis. \\
\hline $\begin{array}{l}\text { Noe \& Haynsworth } \\
\text { (175) }\end{array}$ & Low back pain & 50 & $\begin{array}{l}\text { One } \\
\text { month }\end{array}$ & $\begin{array}{l}\text { Epidural depo-Medrol was compared with aqueous betamethasone } \\
\text { in a retrospective evaluation. }\end{array}$ \\
\hline Lee et al (178) & $\begin{array}{l}\text { Lumbosacral } \\
\text { herniated disc } \\
\text { and spinal } \\
\text { stenosis }\end{array}$ & 58 & 2 years & $\begin{array}{l}\text { It appears that the data of the study is derived from other studies } \\
\text { with } 38 \text { patients in the non-invasive group and } 20 \text { patients in the } \\
\text { epidural injection group. }\end{array}$ \\
\hline Gharibo et al (183) & $\begin{array}{l}\text { Lumbar } \\
\text { radiculitis }\end{array}$ & 42 & 3 weeks & $\begin{array}{l}\text { Evaluation was conducted to look at interlaminar versus } \\
\text { transforaminal epidural steroids for the treatment of subacute } \\
\text { lumbar radicular pain. }\end{array}$ \\
\hline Kapoor et al (193) & $\begin{array}{l}\text { Lumbar } \\
\text { radiculopathy }\end{array}$ & One & NA & $\begin{array}{l}\text { Authors described in a case-report the gadolinium encephalopathy } \\
\text { after intrathecal gadolinium injection. }\end{array}$ \\
\hline
\end{tabular}

carried out utilizing Cochrane review criteria as shown in Tables 10 and 11. Studies achieving Cochrane scores of 9 or higher were considered as high quality, 6 to 8 were considered as moderate quality, and studies scoring less than 6 were excluded.

There were 12 randomized trials $(110,111,133$, $136,139,140,142,144,147,153,179,180,198,199)$ scoring high quality, 8 scored moderate quality $(72,128$, $130,135,141,165,174,181)$, and 3 were of low quality $(129,177,194)$.

Among the fluoroscopically-guided randomized controlled trials 7 were of high quality $(110,111,147,179,180,198,199)$ with 2 duplicate publications $(111,112,199,200), 4$ were of moderate quality $(71,165,174,181)$, and 2 were of low quality $(177,194)$. Among the non-fluoroscopic randomized trials, 7 were of high quality $(133,136,139,140,142,144,153), 4$ were of moderate quality $(128,129,135,141)$, and one was of low quality (129).

A methodological quality assessment of the observational studies meeting inclusion criteria was carried out utilizing Newcastle-Ottawa Scales as illustrated in Table 12. For cohort studies, studies achieving scores of 10 or higher were considered high quality; 7 to 9 were considered moderate quality; studies scoring less than 7 were considered low quality and were excluded. There were no case control studies.

Among the non-randomized studies, the methodological quality assessment indicated no high quality studies, 2 were considered of moderate quality $(145,151)$, and 1 was considered as low quality (182).

\subsection{Meta-Analysis}

Among the 11 randomized trials evaluating the role of epidural injections in disc herniation without fluoroscopy $(128-130,133,135,136,139-142,144) 5$ were placebo control $(130,135,139,140,144)$; one placebo controlled study evaluated the role of epidural injections in spinal stenosis (153). In reference to the active controlled trials, there were 7 trials all evaluating the role of disc herniation $(128,129,133,135,136,141,142)$. There were 3 placebo controlled trials evaluating injection of sodium chloride solution into the interspinous ligament compared with epidural steroid injection $(130,139,144)$. One study assessed paravertebral steroid versus epidural steroid (135). Another study (140) assessed epidural saline versus epidural steroid. The study in spinal stenosis (155) assessed epidural saline versus 


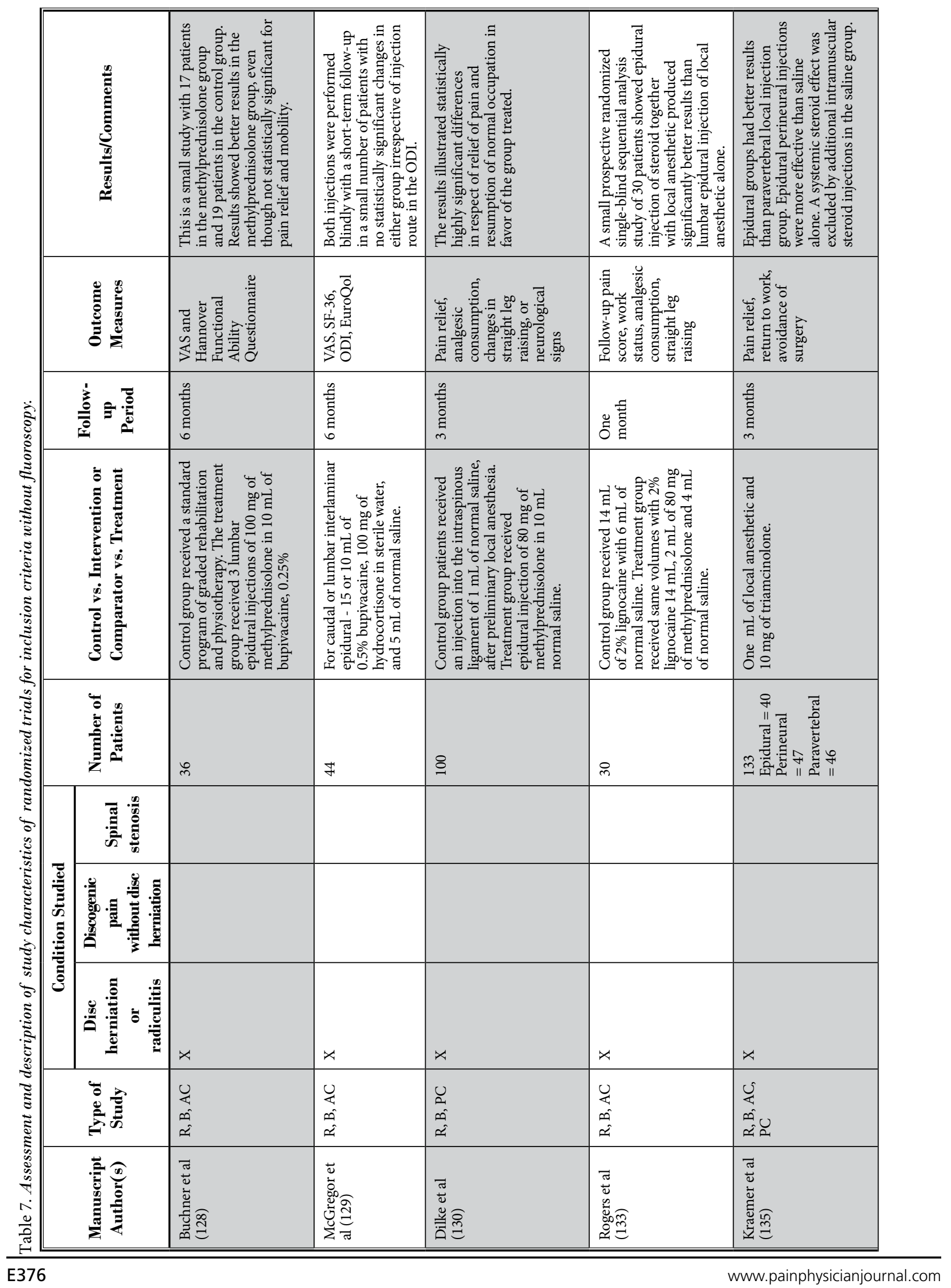




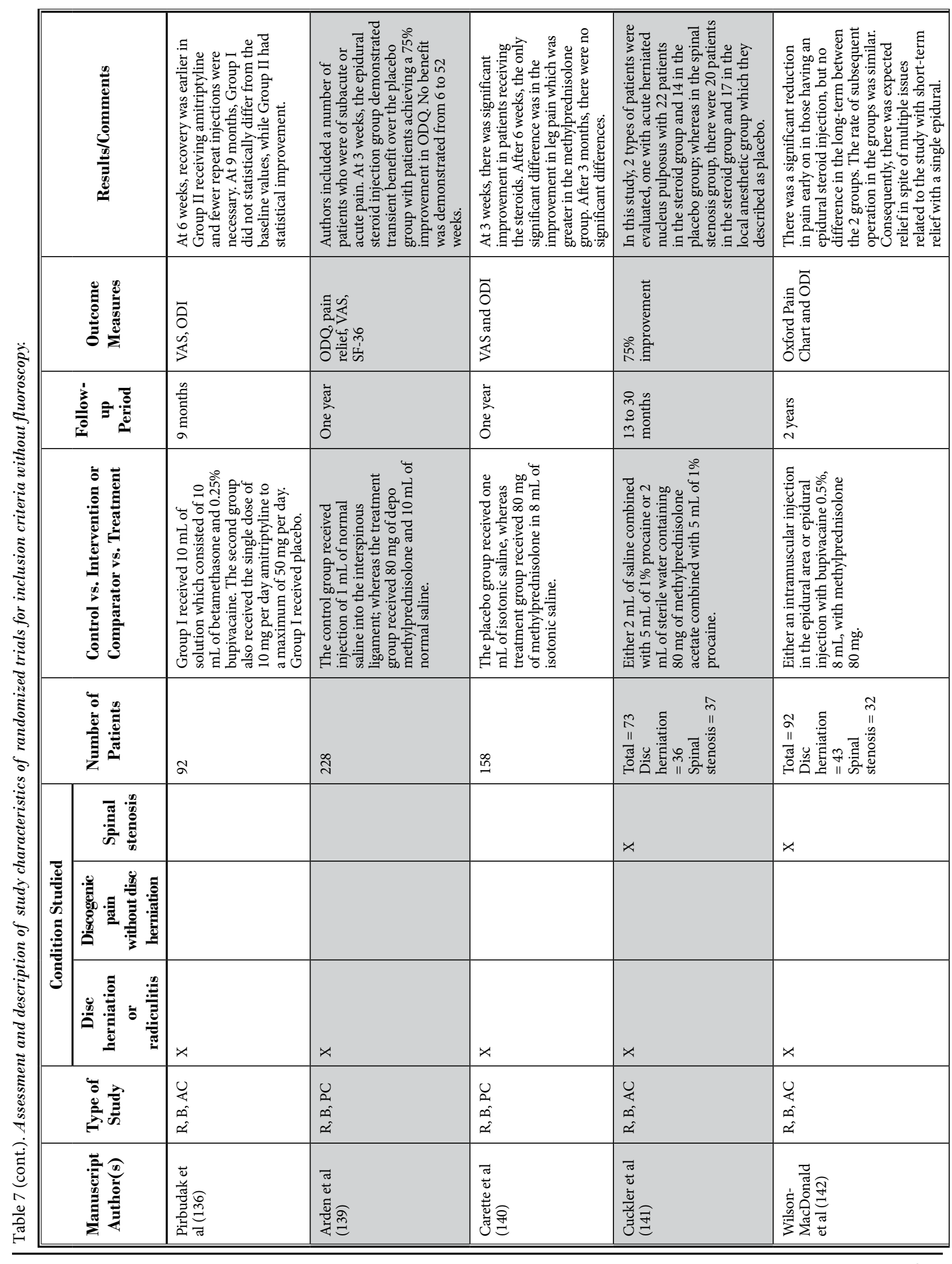



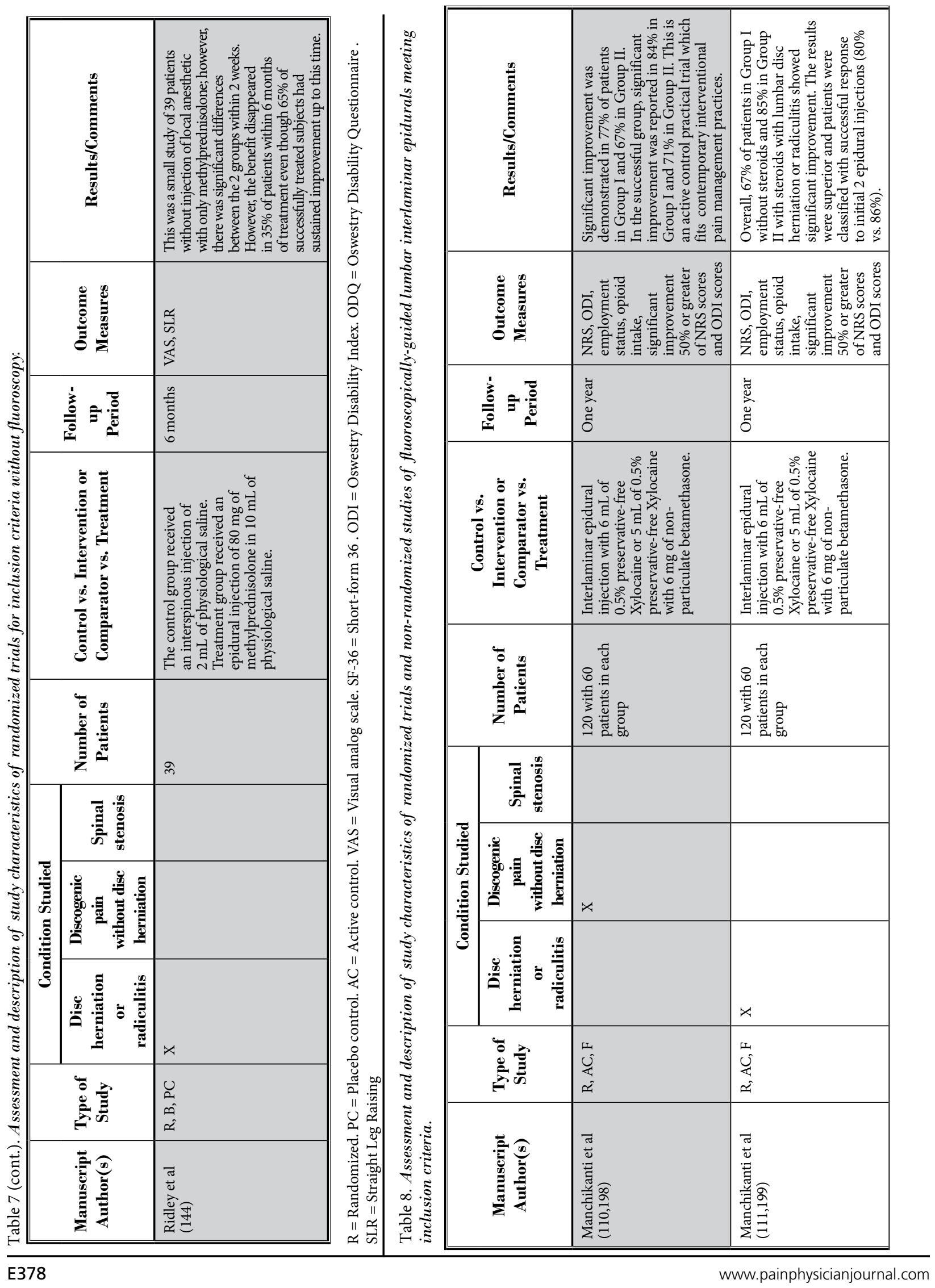


\section{Effectiveness of Lumbar Interlaminar Epidural Injections}

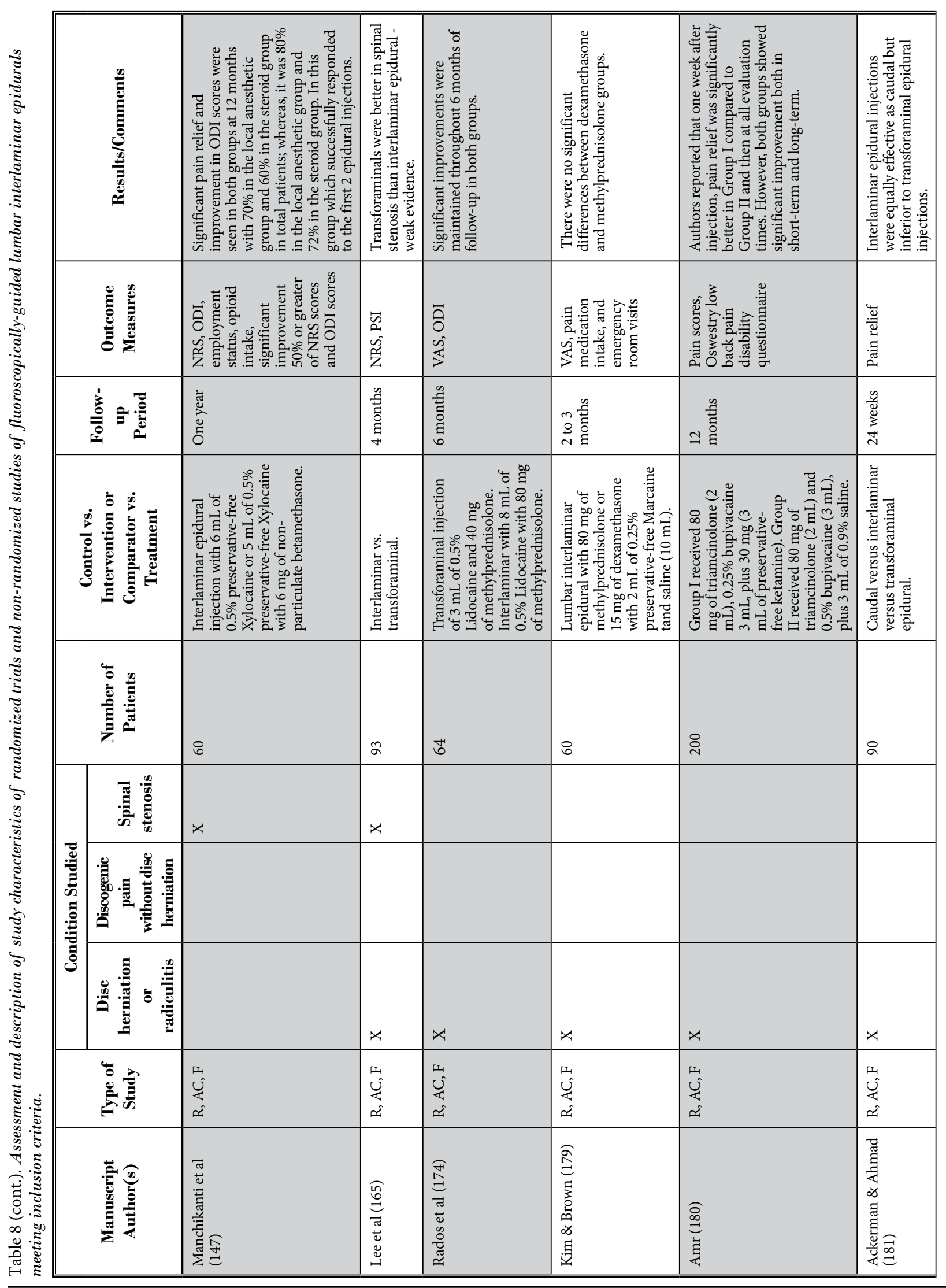




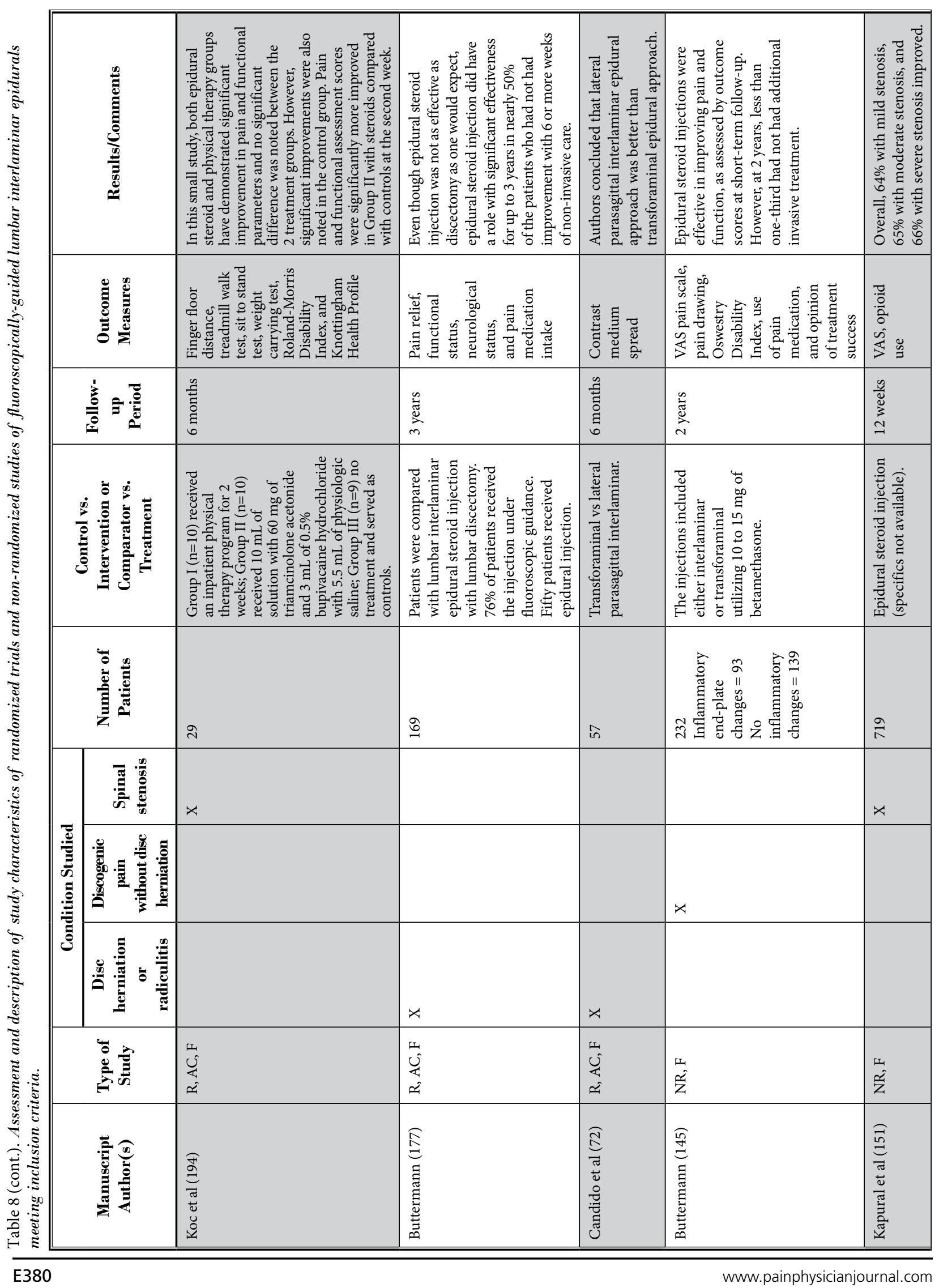


Effectiveness of Lumbar Interlaminar Epidural Injections

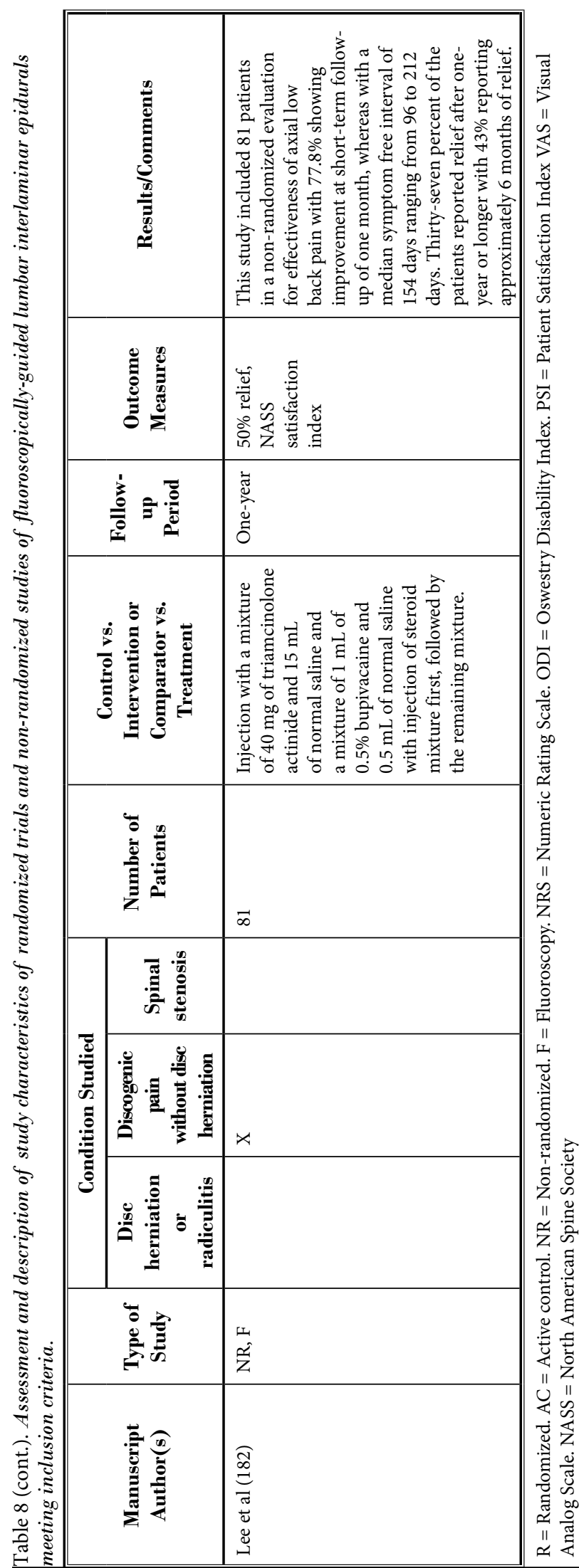

steroid or local anesthetic. Among the active control trials, there were only 2 trials evaluating lidocaine compared with lidocaine with steroid $(133,141)$. The remaining 3 trials were utilizing separate methodology $(128,130,136)$, thus no meta-analysis was possible for non-fluoroscopic studies.

All of the fluoroscopically-guided trials were heterogenous except 2 studies evaluating disc herniation $(72,174)$ assessing the role of transforaminal versus lumbar interlaminar. A total of 8 fluoroscopically-guided randomized trials $(72,111,165,174,177,179-181,199)$ evaluated disc herniation with one duplicate $(111,199)$.

Only one randomized trial evaluated discogenic pain under fluoroscopic guidance $(110,198)$. Three randomized trials evaluated lumbar spinal stenosis under fluoroscopic guidance $(147,165,194)$, whereas 3 studies evaluated without fluoroscopy $(141,142,153)$.

Thus, meta-analysis was not feasible.

\subsection{Study Characteristics}

The study characteristics of the included studies for both randomized trials and non-randomized studies are illustrated in Table 7 and 8.

\subsection{Analysis of Evidence}

The evidence was synthesized based on the specific condition for which lumbar interlaminar epidural injection was provided. Table 13 illustrates the results of randomized trials of the effectiveness of lumbar interlaminar epidural injections in managing disc herniation of radiculitis, Table 14 illustrates effectiveness in managing axial or discogenic pain, and Table 15 illustrates effectiveness in managing spinal stenosis.

\subsubsection{Disc Herniation and Radiculitis}

There were a total of 19 studies meeting the inclusion criteria evaluating lumbar interlaminar epidural injections in managing disc herniation or radiculitis $(72,111,128-130,133,135,136,139-142$, 144,165, $174,177,179-181,199)$ with one duplicate $(111,199)$ (Table 13). Among these, 8 randomized trials were performed under fluoroscopy $(72,111,165,174,177,179-$ $181,199)$ with one duplicate $(111,199)$ and11 trials were performed without fluoroscopy (128$130,133,135,136,139-142,144)$; however, there were no non-randomized evaluations meeting the inclusion criteria. Among the fluoroscopically-guided studies $(72,111,165,174,177,179-181,199)$, there were no placebo controlled evaluations, with all of them being active control trials. Among the studies using a blind 
Table 9. Clinical relevance of included studies.

\begin{tabular}{|c|c|c|c|c|c|c|}
\hline Manuscript Author(s) & $\begin{array}{l}\text { A) Patient } \\
\text { description }\end{array}$ & $\begin{array}{l}\text { B) Description } \\
\text { of interventions } \\
\text { and treatment } \\
\text { settings }\end{array}$ & $\begin{array}{l}\text { C) Clinically } \\
\text { relevant } \\
\text { outcomes }\end{array}$ & $\begin{array}{l}\text { D) Clinical } \\
\text { importance }\end{array}$ & $\begin{array}{l}\text { E) Benefits } \\
\text { versus } \\
\text { potential } \\
\text { harms }\end{array}$ & $\begin{array}{c}\text { Total } \\
\text { Criteria } \\
\text { Met }\end{array}$ \\
\hline \multicolumn{7}{|c|}{ FLUOROSCOPICALLY-GUIDED STUDIES } \\
\hline Candido et al (72) & + & + & + & + & + & $5 / 5$ \\
\hline Manchikanti et al $(110,198)$ & + & + & + & + & + & $5 / 5$ \\
\hline Manchikanti et al $(111,199)$ & + & + & + & + & + & $5 / 5$ \\
\hline Buttermann (145) & + & + & + & + & + & $5 / 5$ \\
\hline Manchikanti et al (147) & + & + & + & + & + & $5 / 5$ \\
\hline Kapural et al (151) & + & + & + & + & + & $5 / 5$ \\
\hline Lee et al (165) & + & - & + & + & - & $3 / 5$ \\
\hline Rados et al (174) & + & + & + & + & + & $5 / 5$ \\
\hline Buttermann (177) & + & + & + & + & + & $5 / 5$ \\
\hline Kim \& Brown (179) & + & + & + & + & + & $5 / 5$ \\
\hline $\operatorname{Amr}(180)$ & + & + & + & + & + & $5 / 5$ \\
\hline Ackerman \& Ahmad (181) & + & + & + & + & + & $5 / 5$ \\
\hline Lee et al (182) & + & + & + & + & + & $5 / 5$ \\
\hline Koc et al (194) & + & + & + & + & + & $5 / 5$ \\
\hline \multicolumn{7}{|c|}{ STUDIES WITHOUT FLUOROSCOPY } \\
\hline Buchner et al (128) & + & + & + & + & + & $5 / 5$ \\
\hline McGregor et al (129) & + & + & - & - & - & $2 / 5$ \\
\hline Dilke et al (130) & + & - & + & - & - & $2 / 5$ \\
\hline Rogers et al (133) & + & + & + & - & + & $4 / 5$ \\
\hline Kraemer et al (135) & + & - & + & - & - & $2 / 5$ \\
\hline Pirbudak et al (136) & + & + & + & + & + & $5 / 5$ \\
\hline Arden et al (139) & + & + & + & + & + & $5 / 5$ \\
\hline Carette et al (140) & + & + & + & - & - & $3 / 5$ \\
\hline Cuckler et al (141) & + & + & + & - & - & $3 / 5$ \\
\hline Wilson-MacDonald et al (142) & + & + & + & - & - & $3 / 5$ \\
\hline Ridley et al (144) & + & + & + & - & - & $3 / 5$ \\
\hline Fukasaki et al (153) & + & + & + & + & + & $5 / 5$ \\
\hline
\end{tabular}

+ = positive; - = negative ; $\mathrm{U}=$ unclear

Scoring adapted from Staal JB, et al. Injection therapy for subacute and chronic low-back pain. Cochrane Database Syst Rev 2008; 3:CD001824 (102). 
Effectiveness of Lumbar Interlaminar Epidural Injections

Table 10. Methodological quality assessment of fluoroscopically-guided randomized trials.

\begin{tabular}{|c|c|c|c|c|c|c|c|c|c|c|c|}
\hline & $\begin{array}{l}\text { Candido } \\
\text { et al } \\
(72)\end{array}$ & $\begin{array}{l}\text { Manchikanti } \\
\text { et al } \\
(110,198)\end{array}$ & $\begin{array}{l}\text { Manchikanti } \\
\text { et al } \\
(111,199)\end{array}$ & $\begin{array}{l}\text { Manchikanti } \\
\text { et al (147) }\end{array}$ & $\begin{array}{l}\text { Lee } \\
\text { et al } \\
(165)\end{array}$ & $\begin{array}{l}\text { Rados } \\
\text { et al } \\
(\mathbf{1 7 4})\end{array}$ & $\begin{array}{c}\text { Buttermann } \\
(177)\end{array}$ & $\begin{array}{l}\text { Kim \& } \\
\text { Brown } \\
(179)\end{array}$ & $\begin{array}{l}\text { Amr } \\
(180)\end{array}$ & $\begin{array}{l}\text { Ackerman } \\
\text { \& Ahmad } \\
(181)\end{array}$ & $\begin{array}{l}\text { Koc } \\
\text { et al } \\
(194)\end{array}$ \\
\hline $\begin{array}{l}\text { Randomization } \\
\text { adequate }\end{array}$ & $\mathrm{Y}$ & $\mathrm{Y}$ & $\mathrm{Y}$ & $\mathrm{Y}$ & $\mathrm{Y}$ & $\mathrm{Y}$ & $\mathrm{N}$ & $\mathrm{Y}$ & $\mathrm{Y}$ & $\mathrm{N}$ & $\mathrm{U}$ \\
\hline $\begin{array}{l}\text { Concealed } \\
\text { treatment } \\
\text { allocation }\end{array}$ & $\mathrm{N}$ & $\mathrm{Y}$ & $\mathrm{Y}$ & $\mathrm{Y}$ & $\mathrm{N}$ & $\mathrm{N}$ & $\mathrm{N}$ & $\mathrm{Y}$ & $\mathrm{Y}$ & $\mathrm{N}$ & $\mathrm{N}$ \\
\hline Patient blinded & $\mathrm{U}$ & $\mathrm{Y}$ & $\mathrm{Y}$ & $\mathrm{Y}$ & $\mathrm{N}$ & $\mathrm{N}$ & $\mathrm{N}$ & $\mathrm{Y}$ & $\mathrm{Y}$ & $\mathrm{N}$ & $\mathrm{N}$ \\
\hline $\begin{array}{l}\text { Care provider } \\
\text { blinded }\end{array}$ & $\mathrm{N}$ & $\mathrm{Y}$ & $\mathrm{Y}$ & $\mathrm{Y}$ & $\mathrm{N}$ & $\mathrm{N}$ & $\mathrm{N}$ & $\mathrm{N}$ & $\mathrm{Y}$ & $\mathrm{N}$ & $\mathrm{N}$ \\
\hline $\begin{array}{l}\text { Outcome } \\
\text { assessor } \\
\text { blinded }\end{array}$ & $\mathrm{U}$ & $\mathrm{N}$ & $\mathrm{N}$ & $\mathrm{N}$ & $\mathrm{N}$ & $\mathrm{N}$ & $\mathrm{N}$ & $\mathrm{N}$ & $\mathrm{N}$ & $\mathrm{N}$ & $\mathrm{N}$ \\
\hline $\begin{array}{l}\text { Drop-out rate } \\
\text { described }\end{array}$ & $\mathrm{Y}$ & $\mathrm{Y}$ & $\mathrm{Y}$ & $\mathrm{Y}$ & $\mathrm{Y}$ & $\mathrm{Y}$ & $\mathrm{Y}$ & $\mathrm{Y}$ & $\mathrm{Y}$ & $\mathrm{Y}$ & $\mathrm{Y}$ \\
\hline $\begin{array}{l}\text { All randomized } \\
\text { participants } \\
\text { analyzed in the } \\
\text { group }\end{array}$ & $\mathrm{Y}$ & $\mathrm{Y}$ & $\mathrm{Y}$ & $\mathrm{Y}$ & $\mathrm{N}$ & $\mathrm{Y}$ & $\mathrm{N}$ & $\mathrm{N}$ & $\mathrm{N}$ & $\mathrm{Y}$ & $\mathrm{N}$ \\
\hline $\begin{array}{l}\text { Reports of } \\
\text { the study free } \\
\text { of suggestion } \\
\text { of selective } \\
\text { outcome } \\
\text { reporting }\end{array}$ & $\mathrm{Y}$ & $\mathrm{Y}$ & $\mathrm{Y}$ & $\mathrm{Y}$ & $\mathrm{Y}$ & $\mathrm{Y}$ & $\mathrm{Y}$ & $\mathrm{Y}$ & $\mathrm{Y}$ & $\mathrm{Y}$ & $\mathrm{Y}$ \\
\hline $\begin{array}{l}\text { Groups similar } \\
\text { at baseline } \\
\text { regarding most } \\
\text { important } \\
\text { prognostic } \\
\text { indicators }\end{array}$ & $\mathrm{U}$ & $\mathrm{N}$ & $\mathrm{N}$ & $\mathrm{N}$ & $\mathrm{Y}$ & $\mathrm{Y}$ & $\mathrm{Y}$ & $\mathrm{Y}$ & $\mathrm{Y}$ & $\mathrm{Y}$ & $\mathrm{Y}$ \\
\hline $\begin{array}{l}\text { Co- } \\
\text { interventions } \\
\text { avoided or } \\
\text { similar }\end{array}$ & $\mathrm{Y}$ & $\mathrm{Y}$ & $\mathrm{Y}$ & $\mathrm{Y}$ & $\mathrm{Y}$ & $\mathrm{Y}$ & $\mathrm{N}$ & $\mathrm{Y}$ & $\mathrm{Y}$ & $\mathrm{Y}$ & $\mathrm{Y}$ \\
\hline $\begin{array}{l}\text { Compliance } \\
\text { acceptable in } \\
\text { all groups }\end{array}$ & $\mathrm{Y}$ & $\mathrm{Y}$ & $\mathrm{Y}$ & $\mathrm{Y}$ & $\mathrm{Y}$ & $\mathrm{Y}$ & $\mathrm{U}$ & $\mathrm{Y}$ & $\mathrm{Y}$ & $\mathrm{Y}$ & $\mathrm{N}$ \\
\hline $\begin{array}{l}\text { Time of } \\
\text { outcome } \\
\text { assessment } \\
\text { in all groups } \\
\text { similar }\end{array}$ & $\mathrm{Y}$ & $\mathrm{Y}$ & $\mathrm{Y}$ & $\mathrm{Y}$ & $\mathrm{Y}$ & $\mathrm{Y}$ & $\mathrm{Y}$ & $\mathrm{Y}$ & $\mathrm{Y}$ & $\mathrm{Y}$ & $\mathrm{Y}$ \\
\hline Score & $7 / 12$ & $10 / 12$ & 10/12 & 10/12 & $7 / 12$ & $8 / 12$ & $4 / 12$ & $9 / 12$ & $10 / 12$ & $7 / 12$ & $5 / 12$ \\
\hline
\end{tabular}

$\mathrm{Y}=$ yes; $\mathrm{N}=$ no; $\mathrm{U}=$ undecided 
Pain Physician: July/August 2012; 15:E363-E404

Table 11. Methodological quality assessment of blind randomized trials without fluoroscopy.

\begin{tabular}{|c|c|c|c|c|c|c|c|c|c|c|c|c|}
\hline & $\begin{array}{c}\text { Buchner } \\
\text { et al } \\
(128) \\
\end{array}$ & $\begin{array}{c}\text { McGregor } \\
\text { et al } \\
(129) \\
\end{array}$ & $\begin{array}{r}\text { Dilke } \\
\text { et al } \\
\text { (130) } \\
\end{array}$ & $\begin{array}{c}\text { Rogers } \\
\text { et al } \\
(133) \\
\end{array}$ & $\begin{array}{c}\text { Kraemer } \\
\text { et al } \\
(\mathbf{1 3 5}) \\
\end{array}$ & $\begin{array}{c}\text { Pirbudak } \\
\text { et al } \\
(136) \\
\end{array}$ & $\begin{array}{c}\text { Arden } \\
\text { et al } \\
(\mathbf{1 3 9}) \\
\end{array}$ & $\begin{array}{c}\text { Carette } \\
\text { et al } \\
(\mathbf{1 4 0 )} \\
\end{array}$ & $\begin{array}{c}\text { Cuckler } \\
\text { et al } \\
(141) \\
\end{array}$ & $\begin{array}{c}\text { Wilson- } \\
\text { MacDonald } \\
\text { et al (142) } \\
\end{array}$ & $\begin{array}{c}\text { Ridley } \\
\text { et al } \\
(\mathbf{1 4 4}) \\
\end{array}$ & $\begin{array}{c}\text { Fukasaki } \\
\text { et al } \\
(153) \\
\end{array}$ \\
\hline $\begin{array}{l}\text { Randomization } \\
\text { adequate }\end{array}$ & $\mathrm{Y}$ & $\mathrm{U}$ & $\mathrm{U}$ & $\mathrm{Y}$ & $\mathrm{U}$ & $\mathrm{Y}$ & $\mathrm{Y}$ & $\mathrm{Y}$ & $\mathrm{U}$ & $\mathrm{Y}$ & $\mathrm{Y}$ & $\mathrm{Y}$ \\
\hline $\begin{array}{l}\text { Concealed } \\
\text { treatment } \\
\text { allocation }\end{array}$ & $\mathrm{N}$ & $\mathrm{U}$ & $\mathrm{U}$ & $\mathrm{Y}$ & $\mathrm{U}$ & $\mathrm{Y}$ & $\mathrm{Y}$ & $\mathrm{Y}$ & $\mathrm{U}$ & $\mathrm{Y}$ & $\mathrm{U}$ & U \\
\hline Patient blinded & $\mathrm{N}$ & $\mathrm{N}$ & $\mathrm{Y}$ & $\mathrm{Y}$ & $\mathrm{Y}$ & $\mathrm{Y}$ & $\mathrm{Y}$ & $\mathrm{Y}$ & $\mathrm{Y}$ & $\mathrm{Y}$ & $\mathrm{Y}$ & $\mathrm{Y}$ \\
\hline $\begin{array}{l}\text { Care provider } \\
\text { blinded }\end{array}$ & $\mathrm{N}$ & $\mathrm{N}$ & $\mathrm{N}$ & $\mathrm{N}$ & $\mathrm{U}$ & $\mathrm{Y}$ & $\mathrm{N}$ & $\mathrm{N}$ & $\mathrm{N}$ & $\mathrm{N}$ & $\mathrm{N}$ & $\mathrm{N}$ \\
\hline $\begin{array}{l}\text { Outcome } \\
\text { assessor } \\
\text { blinded }\end{array}$ & $\mathrm{N}$ & $\mathrm{N}$ & $\mathrm{Y}$ & $\mathrm{U}$ & $\mathrm{U}$ & $\mathrm{U}$ & $\mathrm{Y}$ & $\mathrm{Y}$ & $\mathrm{Y}$ & $\mathrm{Y}$ & $\mathrm{U}$ & $\mathrm{U}$ \\
\hline $\begin{array}{l}\text { Drop-out rate } \\
\text { described }\end{array}$ & $\mathrm{Y}$ & $\mathrm{Y}$ & $\mathrm{Y}$ & $\mathrm{Y}$ & $\mathrm{Y}$ & $\mathrm{Y}$ & $\mathrm{Y}$ & $\mathrm{Y}$ & $\mathrm{Y}$ & $\mathrm{Y}$ & $\mathrm{Y}$ & $\mathrm{Y}$ \\
\hline $\begin{array}{l}\text { All randomized } \\
\text { participants } \\
\text { analyzed in the } \\
\text { group }\end{array}$ & $\mathrm{Y}$ & $\mathrm{N}$ & U & $\mathrm{Y}$ & $\mathrm{U}$ & $\mathrm{U}$ & $\mathrm{Y}$ & $\mathrm{Y}$ & $\mathrm{N}$ & $\mathrm{Y}$ & $\mathrm{Y}$ & $\mathrm{Y}$ \\
\hline $\begin{array}{l}\text { Reports of } \\
\text { the study free } \\
\text { of suggestion } \\
\text { of selective } \\
\text { outcome } \\
\text { reporting }\end{array}$ & $\mathrm{Y}$ & $\mathrm{Y}$ & $\mathrm{Y}$ & $\mathrm{Y}$ & $\mathrm{Y}$ & $\mathrm{Y}$ & $\mathrm{Y}$ & $\mathrm{Y}$ & $\mathrm{Y}$ & $\mathrm{Y}$ & $\mathrm{Y}$ & $\mathrm{Y}$ \\
\hline $\begin{array}{l}\text { Groups similar } \\
\text { at baseline } \\
\text { regarding most } \\
\text { important } \\
\text { prognostic } \\
\text { indicators }\end{array}$ & $\mathrm{N}$ & $\mathrm{Y}$ & $\mathrm{Y}$ & $\mathrm{Y}$ & $\mathrm{Y}$ & $\mathrm{Y}$ & $\mathrm{Y}$ & $\mathrm{Y}$ & $\mathrm{Y}$ & $\mathrm{N}$ & $\mathrm{Y}$ & $\mathrm{Y}$ \\
\hline $\begin{array}{l}\text { Co- } \\
\text { interventions } \\
\text { avoided or } \\
\text { similar } \\
\end{array}$ & $\mathrm{Y}$ & $\mathrm{Y}$ & $\mathrm{Y}$ & $\mathrm{Y}$ & $\mathrm{Y}$ & $\mathrm{Y}$ & $\mathrm{Y}$ & $\mathrm{Y}$ & $\mathrm{Y}$ & $\mathrm{Y}$ & $\mathrm{Y}$ & $\mathrm{Y}$ \\
\hline $\begin{array}{l}\text { Compliance } \\
\text { acceptable in } \\
\text { all groups }\end{array}$ & $\mathrm{Y}$ & $\mathrm{N}$ & $\mathrm{Y}$ & Y & $\mathrm{Y}$ & $\mathrm{Y}$ & $\mathrm{Y}$ & $\mathrm{Y}$ & $\mathrm{Y}$ & $\mathrm{Y}$ & $\mathrm{Y}$ & $\mathrm{Y}$ \\
\hline $\begin{array}{l}\text { Time of } \\
\text { outcome } \\
\text { assessment } \\
\text { in all groups } \\
\text { similar }\end{array}$ & $\mathrm{Y}$ & $\mathrm{Y}$ & $\mathrm{Y}$ & $\mathrm{Y}$ & $\mathrm{Y}$ & $\mathrm{Y}$ & $\mathrm{Y}$ & $\mathrm{Y}$ & $\mathrm{Y}$ & $\mathrm{Y}$ & $\mathrm{Y}$ & $\mathrm{Y}$ \\
\hline Score & $7 / 12$ & $5 / 12$ & $8 / 12$ & $10 / 12$ & $7 / 12$ & 10/12 & $11 / 12$ & $11 / 12$ & $8 / 12$ & 10/12 & $9 / 12$ & $9 / 12$ \\
\hline
\end{tabular}

$\mathrm{Y}=$ yes; $\mathrm{N}=\mathrm{no} ; \mathrm{U}=$ undecided 
Effectiveness of Lumbar Interlaminar Epidural Injections

Table 12. Newcastle-Ottawa quality assessment scale for cohort studies.

\begin{tabular}{|c|c|c|c|}
\hline & $\begin{array}{c}\text { Buttermann } \\
(\mathbf{1 4 5})\end{array}$ & $\begin{array}{c}\text { Kapural et } \\
\text { al (151) }\end{array}$ & $\begin{array}{l}\text { Lee et al } \\
(182)\end{array}$ \\
\hline \multicolumn{4}{|l|}{ Selection } \\
\hline \multicolumn{4}{|l|}{ 1) Representativeness of the exposed cohort } \\
\hline a) truly representative of the average ___ (describe) in the community * & $\mathrm{X}$ & $\mathrm{X}$ & $\mathrm{X}$ \\
\hline \multicolumn{4}{|l|}{ b) somewhat representative of the average pain patients in the community * } \\
\hline \multicolumn{4}{|l|}{ c) selected group of users, e.g. nurses, volunteers } \\
\hline \multicolumn{4}{|l|}{ d) no description of the derivation of the cohort } \\
\hline \multicolumn{4}{|l|}{ 2) Selection of the non exposed cohort } \\
\hline \multicolumn{4}{|l|}{ a) drawn from the same community as the exposed cohort ${ }^{*}$} \\
\hline b) drawn from a different source & $\mathrm{X}$ & $\mathrm{X}$ & $\mathrm{X}$ \\
\hline \multicolumn{4}{|l|}{ c) no description of the derivation of the non exposed cohort } \\
\hline \multicolumn{4}{|l|}{ 3) Ascertainment of exposure } \\
\hline a) secure record (e.g. surgical records) ${ }^{*}$ & $\mathrm{X}$ & $\mathrm{X}$ & $\mathrm{X}$ \\
\hline \multicolumn{4}{|l|}{ b) structured interview * } \\
\hline \multicolumn{4}{|l|}{ c) written self report } \\
\hline \multicolumn{4}{|l|}{ d) no description } \\
\hline \multicolumn{4}{|l|}{ 4) Demonstration that outcome of interest was not present at start of study } \\
\hline \multicolumn{4}{|l|}{ a) yes * } \\
\hline \multicolumn{4}{|l|}{ b) no } \\
\hline \multicolumn{4}{|l|}{ Comparability } \\
\hline 1) Comparability of cohorts on the basis of the design or analysis & $\mathrm{X}$ & $\mathrm{X}$ & \\
\hline \multicolumn{4}{|l|}{ a) study controls for __ (select the most important factor) ${ }^{*}$} \\
\hline \multicolumn{4}{|l|}{$\begin{array}{l}\text { b) study controls for any additional factor }{ }^{*} \text { (This criteria could be modified to indicate } \\
\text { specific control for a second important factor.) }\end{array}$} \\
\hline \multicolumn{4}{|l|}{ Outcome (Exposure) } \\
\hline \multicolumn{4}{|l|}{ 1) Assessment of outcome } \\
\hline \multicolumn{4}{|l|}{ a) independent blind assessment ${ }^{*}$} \\
\hline b) record linkage * & $\mathrm{X}$ & $\mathrm{X}$ & $\mathrm{X}$ \\
\hline \multicolumn{4}{|l|}{ c) self report } \\
\hline \multicolumn{4}{|l|}{ d) no description } \\
\hline \multicolumn{4}{|l|}{ 2) Was follow-up long enough for outcomes to occur } \\
\hline a) yes (select an adequate follow-up period for outcome of interest) * & $\mathrm{X}$ & $\mathrm{X}$ & $\mathrm{X}$ \\
\hline \multicolumn{4}{|l|}{ b) no } \\
\hline \multicolumn{4}{|l|}{ 3) Adequacy of follow-up of cohorts } \\
\hline a) complete follow-up - all subjects accounted for * & $\mathrm{X}$ & $\mathrm{X}$ & $\mathrm{X}$ \\
\hline \multicolumn{4}{|l|}{$\begin{array}{l}\text { b) subjects lost to follow-up unlikely to introduce bias - small number lost -> __ } \\
\text { (select an adequate \%) follow-up, or description provided of those lost)* }\end{array}$} \\
\hline \multicolumn{4}{|l|}{ c) follow-up rate $<\ldots \ldots$ (select an adequate $\%$ ) and no description of those lost } \\
\hline d) no statement & & & \\
\hline SCORE & $7 / 13$ & $7 / 13$ & $6 / 13$ \\
\hline
\end{tabular}

Note: A study can be awarded a maximum of one star for each numbered item within the Selection and Outcome categories. A maximum of two stars can be given for comparability.Wells GA, et al. The Newcastle-Ottawa Scale (NOS) for assessing the quality of nonrandomized studies in meta-analysis. www.ohri.ca/programs/clinical_epidemiology/oxford.asp (103). 
technique without fluoroscopy, 5 were placebo controlled $(130,135,139,140,144)$. Placebo control was inappropriate in some studies and importantly most widely quoted Carette et al's study (140). Dilke et al (130), Arden et al (139), and Ridley et al (144) used appropriate placebo controlled designs either with interspinous injection or intramuscular injection of saline. Others utilized epidural saline, which may not be appropriate, intramuscular steroid injections, or local anesthetic and considered them as placebo controlled. Among the fluoroscopically-guided studies, 2 studies utilized a total of 100 or more patients $(111,180,199)$. Further, only one study $(111,199)$ was carried out utilizing a randomized, active controlled design, providing treatments as needed based on a robust measure of significant improvement considered as $50 \%$ improvement in pain and function with 120 patients with one-year follow-up with the number of injections ranging from 1 to 5 , with significantly better results in the successful group, and performed in contemporary interventional pain management settings. The second study (180) included 200 patients; however, they compared $80 \mathrm{mg}$ of triamcinolone with $30 \mathrm{mg}$ of preservative-free ketamine or $3 \mathrm{~mL}$ of $0.9 \%$ sodium chloride solution, illustrating significant improvement in both groups. Among the non-fluoroscopic evaluations, there were 4 studies with more than 100 patients undergoing interventions $(130,135,139,140)$.

Based on the evaluations separating fluoroscopically-guided versus non-fluoroscopic evaluations, results were positive for short-term relief in 5 trials performed under fluoroscopy $(111,165,174,180,181,199)$; whereas, they were undetermined or not applicable in 3 trials $(72,177,179)$. Consequently all of the trials were positive on a short-term basis. Among the trials evaluating long-term relief, there were 4 trials evaluating relief of 6 months or longer $(111,174,180,181)$ and 2 trials evaluating outcomes for longer than one year $(111,180)$. Among these, 4 trials showed positive results $(111,174,180,181)$; whereas, in 2 trials the results were undetermined or not applicable $(177,179)$. Among the studies evaluating at least a one year follow-up, 2 trials showed positive results $(111,180)$; whereas, 2 trials showed the results which were undetermined or not applicable $(177,179)$.

However, with blind randomized trials, the results were highly mixed due to various issues involved. Some of the issues related to providing only one injection or providing injections of 3 in a series and following through a one-year follow-up. With one injection, one could expect relief of 3 to 4 weeks, however, no more than 3 months. Thus, the follow-up after that does not indicate improvement except for the rare patients who show long-term relief. Some of the studies also had flawed selection criteria. Overall, of 11 randomized trials, 7 of them showed short-term positive results $(128,130,133,135,136,142,144)$ and the remaining 4 showed either undetermined or negative results $(129,139-141)$. However, the results were uniformly negative after 3 months or not able to be determined in all the studies except one (136), which showed positive results comparing prednisone with local anesthetic with or without amitriptyline.

\subsubsection{Effectiveness}

Of the 8 randomized trials meeting the inclusion criteria performed under fluoroscopy $(72,111,165,174,177,179-181,199), \quad 4$ trials showed positive results for short-term relief $(111,165,174,180,181,199)$ with one duplicate $(111,199)$; whereas, in 2 trials, the results were indeterminate or not applicable $(177,179)$. Among the non-fluoroscopic studies, all but one study (136) were negative for longterm relief.

\subsubsection{Axial or Lumbar Discogenic Pain}

Results are illustrated in Table 14 . There were 3 studies meeting the inclusion criteria $(110,145,182,198)$ with one duplicate $(110,198)$. Only one study was randomized, active controlled performed under fluoroscopy $(110,198)$ including 120 patients with one year follow-up showing positive results, both with local anesthetic and steroids performed in a contemporary interventional pain management practice. The other 2 studies $(145,182)$ were non-randomized; however, they were performed under fluoroscopy. There were no placebo-controlled trials evaluating axial or discogenic pain. The only randomized trial also excluded facet joint or sacroiliac joint pain prior to epidural injections and effectiveness $(110,198)$. This trial showed positive results with 60 patients in both groups after exclusion of facet joint or sacroiliac joint pain. This was a large trial in a contemporary interventional pain management practice with an active controlled design showing positive results. Among the 2 non-randomized trials, one study (182) showed positive results at 3 and 6 months; however, the results were unable to be determined at 12 months due to the injections being performed one to 3 not based on return of pain. In the second non-randomized study (145), the results were confusing; thus, 


\section{Effectiveness of Lumbar Interlaminar Epidural Injections}

\begin{tabular}{|c|c|c|c|c|c|c|c|c|c|c|}
\hline & \multicolumn{2}{|l|}{ 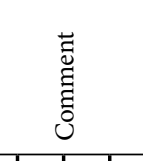 } & 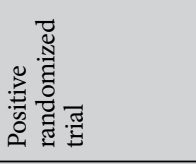 & 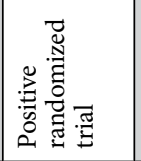 & 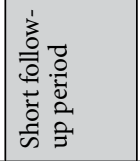 & 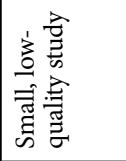 & 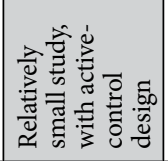 & 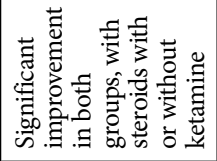 & 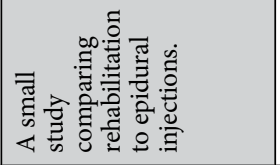 & 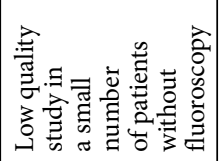 \\
\hline \multirow{9}{*}{ 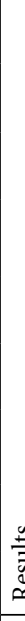 } & \multirow{6}{*}{ 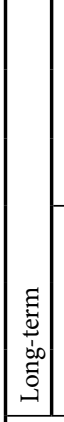 } & 灾 & $\overleftrightarrow{z}$ & $\overleftrightarrow{z}$ & $\overleftrightarrow{z}$ & $D$ & $\overleftrightarrow{z}$ & $\overleftrightarrow{z}$ & $\overleftrightarrow{z}$ & $\overleftrightarrow{z}$ \\
\hline & & $\leftrightarrows$ & a & $\overleftrightarrow{z}$ & $\overleftrightarrow{z}$ & $D$ & $\overleftrightarrow{z}$ & 志 & 艺 & $\overleftrightarrow{z}$ \\
\hline & & 5 & $\infty$ & $\overleftrightarrow{z}$ & $\overleftrightarrow{z}$ & $D$ & $\overleftrightarrow{z}$ & z & 艺 & $\overleftrightarrow{z}$ \\
\hline & & 空 & $\overleftrightarrow{z}$ & $\overleftrightarrow{z}$ & $\overleftrightarrow{z}$ & $D$ & $\mathbb{z}$ & 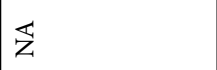 & 忞 & z \\
\hline & & 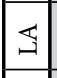 & $a$ & $\overleftrightarrow{z}$ & $\overleftrightarrow{z}$ & $D$ & $\overleftrightarrow{z}$ & 太 & $z$ & $\overleftrightarrow{z}$ \\
\hline & & 5 & 2 & 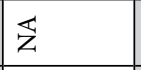 & 2 & $D$ & $\overleftrightarrow{z}$ & $z$ & 2 & $z$ \\
\hline & \multirow{2}{*}{ 章 } & 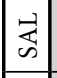 & $\overleftrightarrow{z}$ & $\overleftrightarrow{z}$ & $\overleftrightarrow{z}$ & $D$ & $\overleftrightarrow{z}$ & $\overleftrightarrow{z}$ & 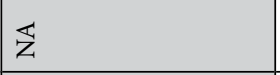 & $z$ \\
\hline & & 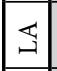 & 2 & $\overleftrightarrow{z}$ & $\overleftrightarrow{z}$ & $D$ & $\overleftrightarrow{z}$ & 点 & $z$ & $\overleftrightarrow{z}$ \\
\hline & 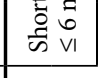 & 5 & 2 & 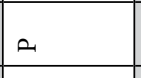 & D & $D$ & $\overleftarrow{z}$ & $z$ & a & $z$ \\
\hline & $\begin{array}{l}\dot{0} \\
\stackrel{\Xi}{\Xi} \\
\stackrel{\Xi}{\beth}\end{array}$ & & 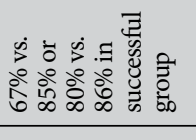 & 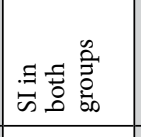 & $\overleftrightarrow{z}$ & $D$ & $P$ & 竡总 & $\overleftrightarrow{z}$ & $\mathbb{z}$ \\
\hline & $\begin{array}{l}\text { b } \\
\text { ह } \\
6 \\
\end{array}$ & & 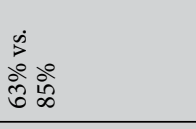 & 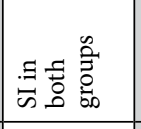 & 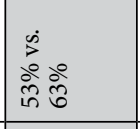 & $D$ & $\overleftrightarrow{z}$ & 音产 & 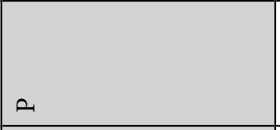 & $\overline{\tilde{z}}$ \\
\hline 害 & $\begin{array}{l}\dot{s} \\
\text { : } \\
m \\
\end{array}$ & & 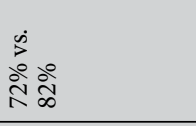 & 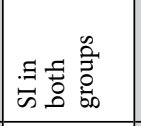 & 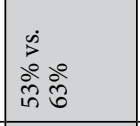 & $D$ & $\overleftrightarrow{z}$ & 总言 & $a$ & $\overrightarrow{\tilde{z}}$ \\
\hline & 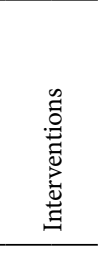 & & 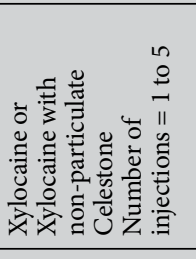 & 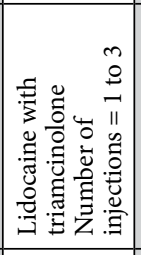 & 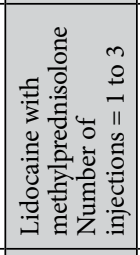 & 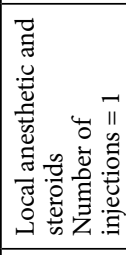 & 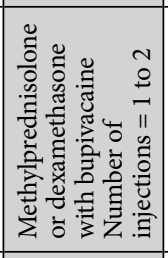 & 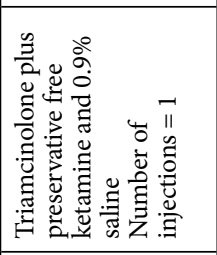 & 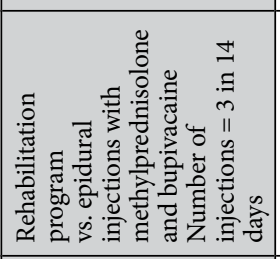 & 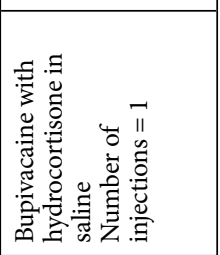 \\
\hline & 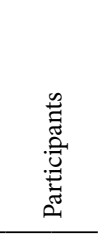 & & 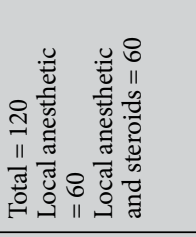 & 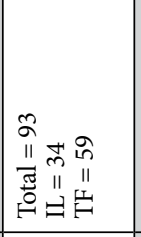 & 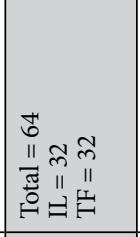 & 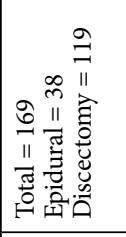 & 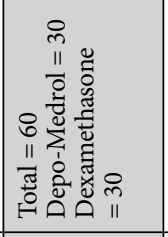 & 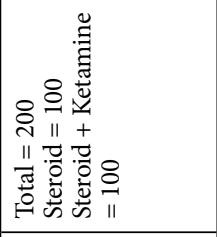 & 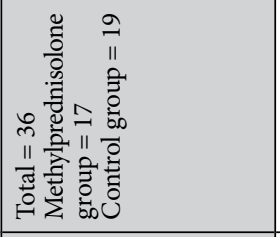 & 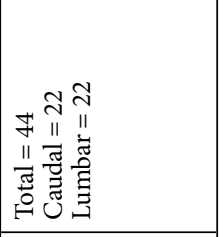 \\
\hline & 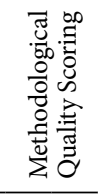 & & $\stackrel{\approx}{0}$ & $\frac{2}{n}$ & $\frac{\pi}{\infty}$ & $\stackrel{7}{7}$ & $\frac{2}{2}$ & $\stackrel{\Xi}{\Xi}$ & $\underset{1}{\mathbb{N}}$ & $\stackrel{2}{15}$ \\
\hline & 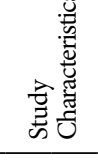 & & 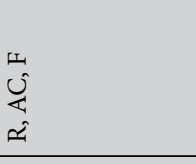 & 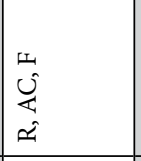 & 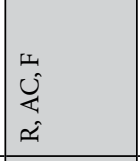 & 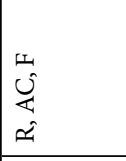 & 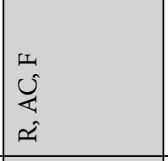 & 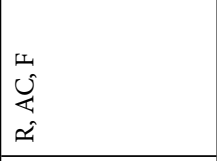 & \begin{tabular}{|l}
0 \\
4 \\
an \\
$\approx$ \\
\end{tabular} & 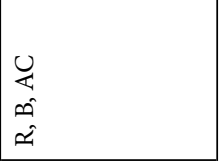 \\
\hline & 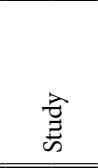 & & 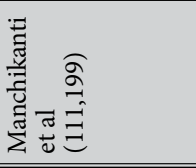 & 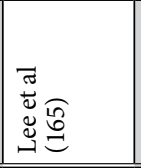 & 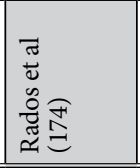 & 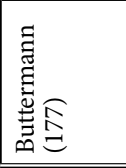 & 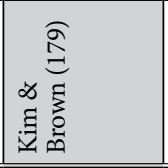 & 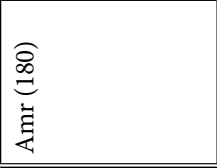 & 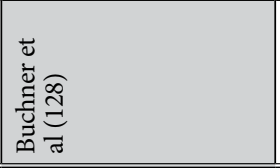 & 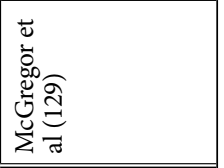 \\
\hline
\end{tabular}


Pain Physician: July/August 2012; 15:E363-E404

\begin{tabular}{|c|c|c|c|c|c|c|c|c|c|}
\hline & \multicolumn{2}{|l|}{ 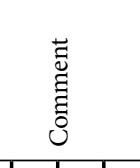 } & 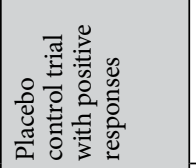 & 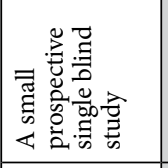 & 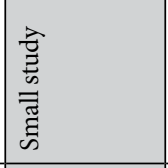 & 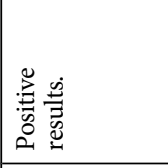 & 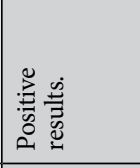 & 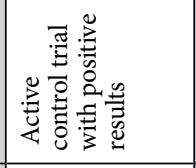 & 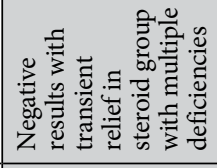 \\
\hline & \multirow{6}{*}{ 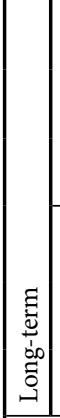 } & 离 & $\overleftrightarrow{z}$ & $\mathbb{z}$ & 妾 & $\mathbb{z}$ & $\mathbb{z}$ & 艺 & z \\
\hline & & 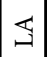 & $\overleftrightarrow{z}$ & $\overleftrightarrow{z}$ & 艺 & 妾 & 妾 & 袁 & $\mathbb{z}$ \\
\hline & & $\dot{s}$ & $\overleftrightarrow{z}$ & $\overleftrightarrow{z}$ & $\overleftrightarrow{z}$ & 㐏 & $\overleftrightarrow{z}$ & a & $z$ \\
\hline & & $\vec{b}$ & $\overleftrightarrow{z}$ & 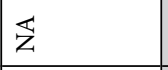 & $\overleftrightarrow{z}$ & 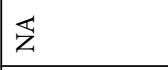 & $\overleftrightarrow{z}$ & $\overleftrightarrow{z}$ & $z$ \\
\hline & & 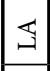 & 艺 & 㐏 & $\overleftrightarrow{z}$ & 艺 & $\overleftrightarrow{z}$ & $\underset{\star}{\star}$ & $\overleftrightarrow{z}$ \\
\hline & & $\omega$ & $\overleftrightarrow{z}$ & $\overleftrightarrow{\mathrm{z}}$ & $\overleftrightarrow{\mathrm{z}}$ & A & $\overleftrightarrow{\mathrm{z}}$ & a & $z$ \\
\hline & \multirow{3}{*}{ 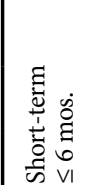 } & $\vec{\infty}$ & 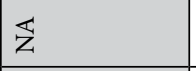 & $\overleftrightarrow{z}$ & $\overleftrightarrow{z}$ & 艺 & $\overleftrightarrow{z}$ & $\overleftrightarrow{z}$ & $z$ \\
\hline & & $\Xi$ & 艺 & 2 & 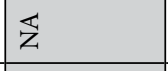 & 艺 & 艺 & \begin{tabular}{|l} 
态 \\
究
\end{tabular} & 艺 \\
\hline & & $\xi$ & 2 & A & $\overleftrightarrow{z}$ & $\infty$ & 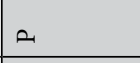 & D & $z$ \\
\hline & $\begin{array}{l}\dot{b} \\
\stackrel{\Xi}{\Xi} \\
\end{array}$ & & $\overleftarrow{z}$ & 艺 & 乙 & 妾 & 艺 & 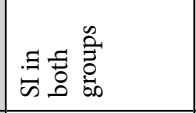 & $\overrightarrow{\tilde{z}}$ \\
\hline & $\begin{array}{l}0^{\circ} \\
\text { छ } \\
0\end{array}$ & & $\overleftarrow{z}$ & $\mathbb{z}$ & z & $\infty$ & $\mathbb{z}$ & 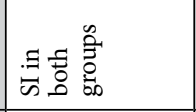 & $\overrightarrow{\bar{z}}$ \\
\hline & 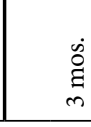 & & 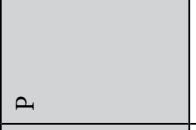 & o & z & $\infty$ & 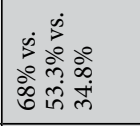 & 恶吾产 & $\overrightarrow{\bar{z}}$ \\
\hline & 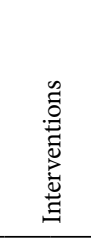 & & 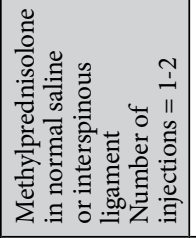 & 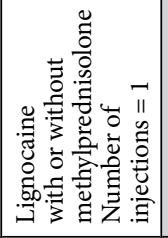 & 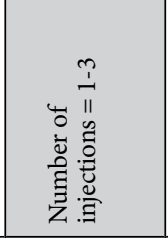 & 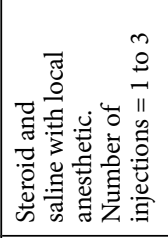 & 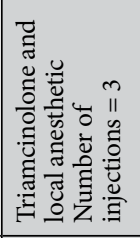 & 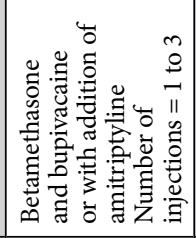 & 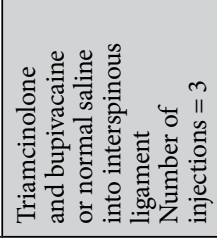 \\
\hline & 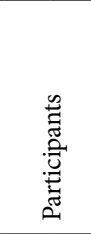 & & 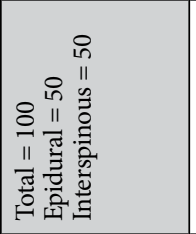 & 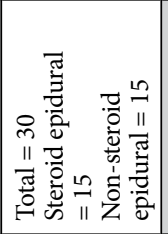 & 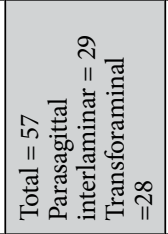 & 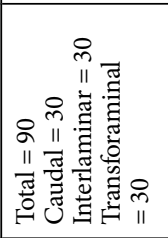 & 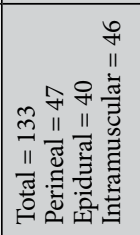 & 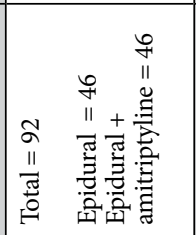 & 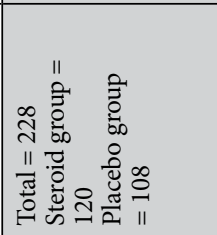 \\
\hline & 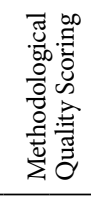 & & $\underset{\infty}{\approx}$ & $\stackrel{\Xi}{\Xi}$ & $\frac{Z}{\pi}$ & $\stackrel{Z}{i}$ & $\frac{\mathfrak{Z}}{\mathfrak{1}}$ & $\frac{2}{0}$ & $\stackrel{\Xi}{\Xi}$ \\
\hline & 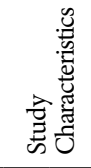 & & $\begin{array}{l}0 \\
0 \\
\infty \\
\alpha \\
\approx\end{array}$ & 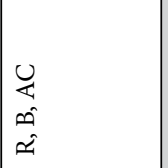 & 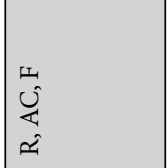 & 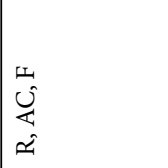 & 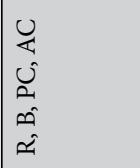 & 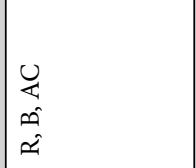 & $\begin{array}{l}0 \\
0 \\
\infty \\
\infty \\
\alpha\end{array}$ \\
\hline & 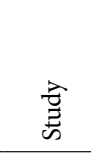 & & 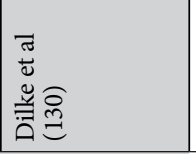 & 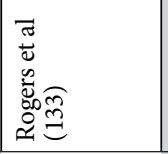 & 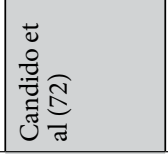 & 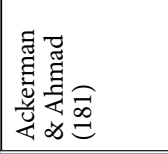 & 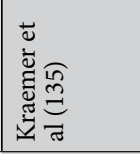 & 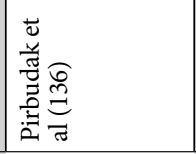 & 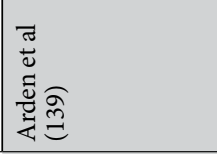 \\
\hline
\end{tabular}


Effectiveness of Lumbar Interlaminar Epidural Injections

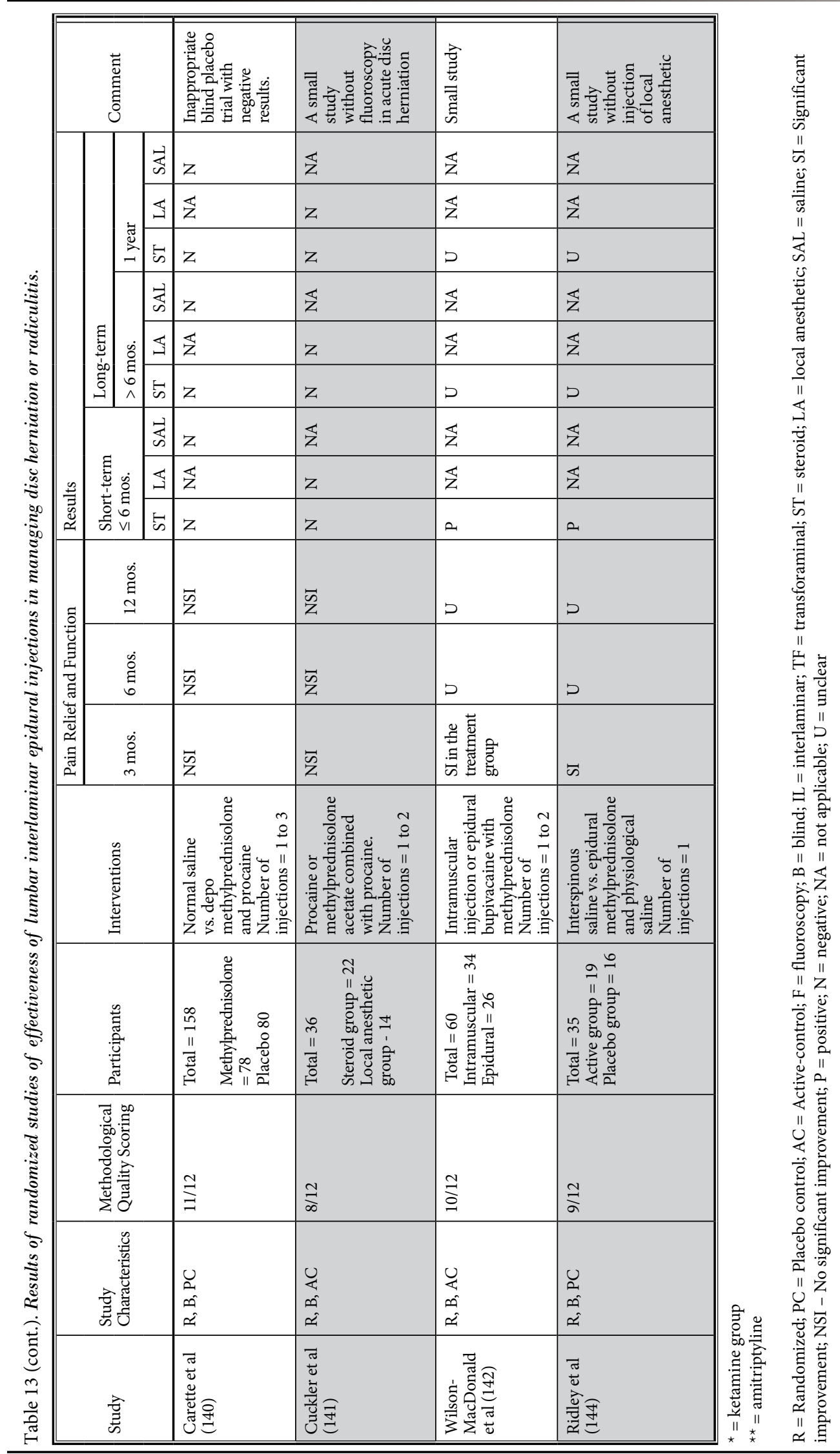


Pain Physician: July/August 2012; 15:E363-E404

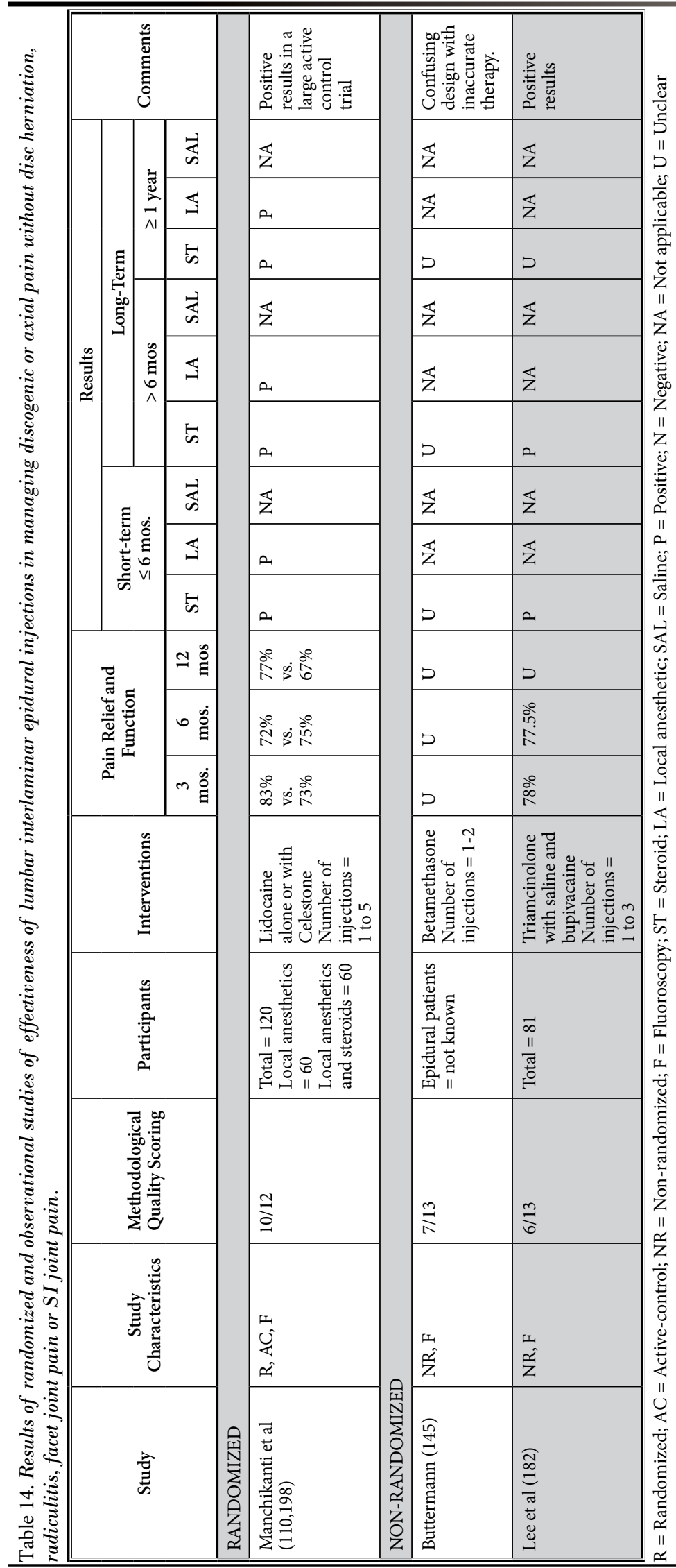

they were classified as undetermined.

\subsubsection{Effectiveness}

Of the one randomized trial $(110,198)$ and 2 non-randomized studies $(145,182)$ the randomized trial and one non-randomized study showed positive results for both short-term and long-term. The third study which was non-randomized (145) showed undetermined results with a confusing design. Only one study evaluated the patients at 12 months with 120 patients $(110,198)$. This study was positive both in short-term and long-term.

\subsubsection{Spinal Stenosis}

Table 15 shows results of randomized and observational studies of effectiveness of lumbar epidural injections in managing spinal stenosis.

There were 6 randomized trials $(141,142,147,155,165,194)$ and one nonrandomized study (151) evaluating the effectiveness of lumbar interlaminar epidural injections in spinal stenosis. However, none of the well-conducted studies utilized 100 or more patients. There were 3 randomized trials performed under fluoroscopy $(147,165,194)$. The study by Manchikanti et al (147) was a preliminary report showing positive results with local anesthetic as well as steroids for central stenosis in a contemporary interventional pain management practice. The other 2 fluoroscopically-guided trials $(165,194)$ and one non-randomized study (151) showed short-term positive results. On a long-term basis, the results were also positive for 6 months or longer in 2 studies $(147,194)$. However, the results were mixed in the groups using a blind technique. One study (142) utilized the intermuscular injection for control with steroids and considered it also as a placebo. Short-term results were positive with blind epidural for spinal stenosis with a small number of patients in one trial (142).

\subsubsection{Effectiveness}

There were 3 randomized trials 
$(147,165,194)$ evaluating spinal stenosis under fluoroscopy with all 3 of them showing positive results. However, only one study by Manchikanti et al (147) evaluated long-term follow-up with positive results. The non-randomized trial also performed under fluoroscopy (151) was positive in short-term.

Among the randomized trials, only the study with a small number of patients by Wilson-McDonald et al (142) was positive for short-term relief.

\subsection{Level of Evidence}

Based on the USPSTF criteria, the evidence is considered at 3 levels - good, fair, or limited.

\subsubsection{Lumbar Disc Herniation}

For lumbar disc herniation with radiculitis, based on 5 of 8 positive randomized trials performed under fluoroscopy the evidence is good for short-term and long-term relief with steroids and fair with local anesthetic.

Considering the blind trials without fluoroscopy, the evidence continues to be good for short-term relief with positive results in 7 of the 11 studies with local anesthetic and steroids. However, the level of evidence based only on the 4 trials showing negative or undetermined results some of which are placebo controlled, is poor to fair. Similarly, for long-term relief, the results in the majority of the studies were negative or undetermined with positive results in only one trial with poor evidence.

Overall, results are positive with good evidence when performed utilizing contemporary interventional pain management techniques with measures of pain and function and repeating them only based on the return of pain with local anesthetic and steroids; however, the evidence is fair when they are performed with only local anesthetic.

\subsubsection{Axial or Lumbar Discogenic Pain}

For axial or lumbar discogenic pain, based on one of one positive randomized trial $(110,198)$ performed under fluoroscopy, the evidence is considered fair for short-term and long-term relief with steroids or with local anesthetic.

\subsubsection{Spinal Stenosis}

For spinal stenosis, based on 3 of 3 positive randomized trials and one positive non-randomized study performed under fluoroscopy, the evidence is considered fair for short-term and long-term relief with local anesthetic and steroids.

\subsubsection{Summary of Evidence}

In summary, the evidence is good for radiculitis secondary to disc herniation with local anesthetics and steroids, fair with local anesthetic only; whereas, it is fair secondary to spinal stenosis with local anesthetic and steroids, and fair for axial pain without disc herniation and with local anesthetic with or without steroids.

\subsection{Complications}

The commonly described complications of interlaminar epidural injections are related either to the needle placement or drug administration (1,46,47,50-59). Multiple infectious complications including epidural abscess, meningitis, and osteomyelitis/discitis have been reported (200-209). One potentially serious complication of the epidural injection is epidural hematomas in patients with or without evidence of any bleeding tendency, anticoagulation, or traumatic needle insertion (210-216). Neurological injuries, though rare, could be devastating related to needle trauma, intraarticular injection, toxic effects of steroids, bleeding, and infection (74,200-227). Other complications include increased pain, seizures, chemical meningitis, dural puncture, disc puncture, subdural air, pneumocephalus, transient blindness, retinal necrosis, chorioretinopathy, hiccups, flushing, and arterial gas embolism $(200,228-245)$. The major theoretical complications of corticosteroid administration include suppression of pituitary adrenal axis, hypercorticism, Cushing's syndrome, osteoporosis, avascular necrosis of the bone, steroid myopathy, epidural lipomatosis, weight gain, fluid retention, and hyperglycemia $(206,217,218,246-251)$.

Manchikanti et al (251) in evaluating 10,000 fluoroscopically-guided epidural injections showed intravascular and return of blood in $0.5 \%$, profuse bleeding and dural puncture in $0.8 \%$, local hematoma and transient nerve root irritation in $0.28 \%$, postlumbar puncture headache in $0.07 \%$, and facial flushing in $0.13 \%$ in lumbar interlaminar epidural injections.

Finally, radiation exposure is also a potential problem with damage to eyes, skin, and gonads (252-254).

\subsection{Discussion}

This systematic review of fluoroscopically-guided and blind lumbar interlaminar epidural injections in managing chronic low back pain and lower extremity pain of disc herniation or radiculitis indicated good evidence for procedures performed under fluoroscopy. 
Pain Physician: July/August 2012; 15:E363-E404

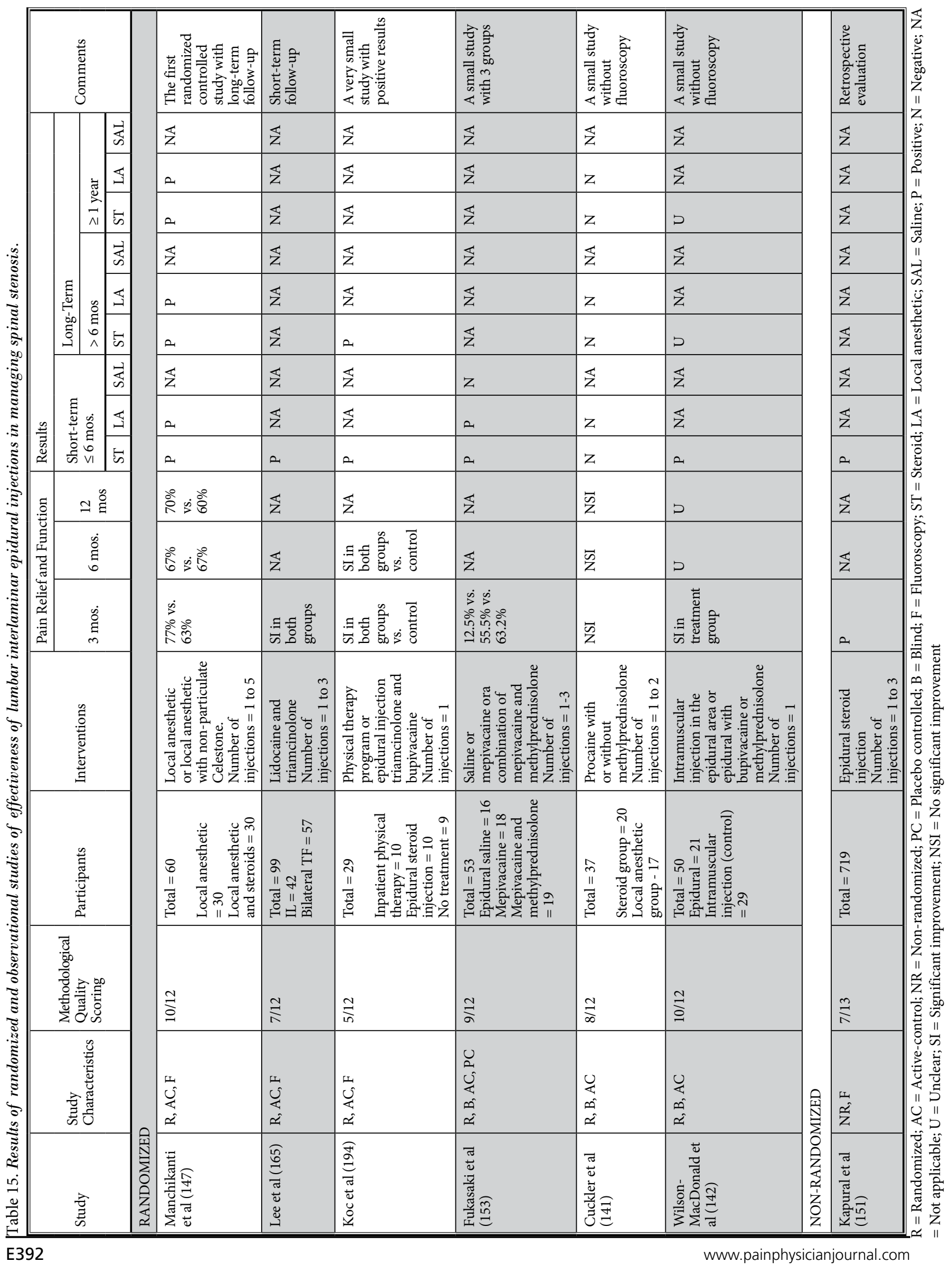


The evidence is fair for spinal stenosis and discogenic or axial pain due to a paucity of literature. We have not evaluated the evidence for lumbar postlaminectomy syndrome as this is not a commonly performed procedure and is considered unsafe with an interlaminar approach. However, for blind lumbar epidural injections the evidence is highly variable and consistently inferior to fluoroscopically-guided epidural injections with local anesthetic and steroids; and evidence is fair for local anesthetic only.

Thus, in addition to the paucity of available fluoroscopic literature meeting inclusion criteria, all of the included non-fluoroscopic studies followed flawed methodology without target delivery of steroids, performing the procedures frequently between $L 3 / 4$ and occasionally $L 4 / 5$ in the lateral position, with poor assessment of outcomes application and analysis. The disadvantages of this approach without fluoroscopy include dilution of the injectate, extra epidural placement of the needle, intravascular placement of the needle, preferential cranial flow of the solution, preferential posterior flow of the solution, difficult placement (with increased risks in postsurgical patients), difficult placement below L4L5 interspace, deviation of needle to non-dependent side, dural puncture, and trauma to the spinal cord. These disadvantages and potential flaws may be avoided with fluoroscopy.

The ultimate results of this systematic review are in stark contrast to previous systematic reviews and guidelines. However, in this evaluation we attempted to evaluate the evidence separately for procedures performed under fluoroscopy for disc herniation and radiculitis, spinal stenosis, and chronic axial or discogenic pain; whereas, others have evaluated by combining multiple conditions and multiple techniques (caudal and interlaminar), fluoroscopically-guided and non-fluoroscopic into one category.

The evidence here is similar compared to caudal epidurals or transforaminal epidurals with or without steroids. Further, when the injections were performed under fluoroscopy, interlaminar epidurals, similar to caudal epidurals, showed superior results in all conditions, including axial or discogenic pain without disc herniation and spinal stenosis.

The debate concerning lumbar epidural steroid injections has been nurtured since the 1970s $(1,15,20,26,46,48,49,51-53,56,59,96,102,255)$. The first systematic review of the effectiveness of caudal epidural steroid injections was performed by Kepes and Duncalf in 1985 (51). They concluded that the rationale for epidural and systematic steroids was not proven. However, in 1986, Benzon (59), utilizing the same studies, concluded that mechanical causes of low back pain, especially those accompanied by signs of nerve root irritation, may respond to epidural steroid injections.This illustrates that systematic reviews have provided different results based on the evaluators.

Bogduk et al (26) extensively studied caudal, interlaminar, and transforaminal epidural injections, including all the literature available at the time, and concluded that the balance of published evidence supports the therapeutic use of caudal epidurals. In 1995, Koes et al (52) reviewed 12 trials of lumbar and caudal epidural steroid injections and reported positive results from only 6 studies. However, review of their analysis showed that there were 5 studies for caudal epidural steroid injections and 7 studies for lumbar epidural steroid injections. Four of the 5 studies involving caudal epidural steroid injections were positive; whereas, 5 of 7 studies for lumbar interlaminar were negative. Their updated analysis (53) with the inclusion of 15 trials also arrived at the same conclusions with inappropriate allocation of the procedures. Multiple other investigators $(52,56,102)$ also have provided differing conclusions. In general, criticism against systematic reviews in the past has been directed toward methodology, small size of the study populations, and other limitations, including long-term follow-up and outcome parameters of the available literature. Further, the paucity of literature has been a factor in the systematic evaluation of evidence for the effectiveness of epidural injections $(15,19,20,27,28,102)$.

Target site concentration of the administered drug including steroids depends on multiple injection variables including the route of administration. Interlaminar epidural injections are considered to be non-specific. Steroids may be prevented from migrating from the posterior epidural space to the anterior or ventral epidural space by the presence of epidural ligaments or scar tissue, with interlaminar administration. The extra epidural placement of the needle, which may go unrecognized without fluoroscopic guidance, is of paramount importance with the interlaminar approach $(61,63,66,67,68,256-260)$. Other disadvantages of the interlaminar approach include erroneous placement of the needle, which may miss the targeted interspace without fluoroscopic guidance; preferential cranial flow of the solution in the epidural space; deviation of the needle to the non-dependent side; difficulty entering the epidural space and delivery of injectate below 
L5, for S1 nerve root involvement; potential risk of dural puncture and post lumbar puncture headache; and finally, the rare, but serious, risk of spinal cord trauma $(66,63,66,67,68,256-260)$. It is a well-known fact that disc herniation mostly involves L4-L5 and L5- S1 discs and the preferential flow to higher levels by placing the needle at L3-4 obviates the entire philosophy of target delivery. Advocates of fluoroscopic guidance point to several studies which have shown that in as many as $30 \%$ of the lumbar epidural injections by experienced injectionists, the epidural space was misidentified $(66,67,73,256-259)$. In fact, Botwin et al (73) in their prospective evaluation of epidurography contrast patterns in fluoroscopically-guided lumbar interlaminar epidural injections found that dorsal contrast spread occurred in all patients, whereas ventral spread was present in only $36 \%$ of the patients. In addition, they also showed that the mean number of vertebral levels of cephalad spread was 1.28 and caudal spread was 0.88 . In another study (61), the spread was unilateral $45 \%$ of the time and the contrast spread was anterior only $43 \%$ to $51 \%$ of the time based on the needle position, indicating over $49 \%$ of the time it was posterior.

In this evaluation, a total of 11 randomized trials and 3 non-randomized studies under fluoroscopy were included. However, randomized blind studies were also included. Only the studies meeting at least moderate quality criteria were included in analysis. The quality assessment of all the manuscripts was performed. This review yielded results different from Parr et al (46) published in 2009, the critical review of APS guidelines (28), and the reassessment of the American College of Occupational and Environmental Medicine (ACOEM) guidelines (49). However, these results still do not correlate with results by Chou and Huffman (20) and Staal et al $(15,102)$. Further, results provided by other reviewers are also in line with the evidence from this review $(85,255,261)$.

Peterson and Hodler (261) in their evaluation of evidence-based radiology, evaluating the evidence for use of therapeutic injections for the spine and sacroiliac joints, concluded that caudal epidural steroid injections were superior. Further, the guidelines for the American Society of Anesthesiologists (ASA) and the American Society of Regional Anesthesia in Pain Medicine (ASRA) also provided favorable evidence.

However, Chou and Huffman (20), Staal et al $(15,102)$, ACOEM guidelines (49), and guidelines from American Academy of Neurology (AAN) (56) provided different conclusions. Chou and Huffman (20) in their evaluation stated that most placebo-controlled trials evaluated either the interlaminar or caudal approach. They combined interlaminar or translaminar epidural injections and caudal epidural injections into one category, and therefore reached erroneous conclusions that these treatments were only effective for short-term relief in radiculopathy.

Staal et al $(15,102)$ evaluated all epidural injections in combination which included caudal, lumbar interlaminar, and lumbar transforaminal as one category. They also failed to separate the response to herniation, stenosis, postlaminectomy syndrome, or discogenic pain, consequently reaching inappropriate conclusions. Thus, the present systematic review contradicts this evidence.

ASA and the ASRA guidelines (255), utilizing a combined approach with physician consensus and systematic review, also recommend epidural steroid injections.

The current systematic review shows that lumbar epidural steroid injections, when appropriately performed, should result in significant improvement in pain and function.

Placebo-controlled neural blockade is not realistic. It has been misinterpreted as most placebo solutions injected into active structures result in active effects $(140,262-274)$. The underlying mechanism of action of epidurally administered steroid and local anesthetic injection is still not well understood. It is believed that the achieved neural blockade alters or interrupts nociceptive input, the reflex mechanism of the afferent fibers, self-sustaining activity of the neurons, and the pattern of central neuronal activities $(1,218)$. Further, corticosteroids have been shown to reduce inflammation by inhibiting either the synthesis or release of a number of pro-inflammatory mediators and by causing a reversible local anesthetic effect (275-279). Local anesthetics also have been described to provide short- to long-term symptomatic relief based on alteration of various mechanisms including excess nociceptive process, excess release of neurotransmitters, nociceptive sensitization of the nervous system, and phenotype changes (278-285). The prolonged effect of local anesthetics in epidural injections and facet joint nerve blocks has been demonstrated in multiple studies (114-116,119,120,147,284,286-292). Sato et al (293) evaluated the prolonged analgesic effect of epidural bupivacaine in a rat model of neuropathic pain with repetitive administration, possibly by inducing a plastic change in nociceptive input. Further, Tachihara et al (294) showed in rats that nerve root infiltration pre- 
vented mechanical allodynia; however, no additional benefit from using corticosteroid was identified.

Further discussions with regards to the superiority of caudal epidurals over either transforaminal epidural injections or interlaminar epidural injections are not proven by this systematic review. This systematic review, however, shows the ability of caudal epidural injections to prevent surgical interventions.

The results of this systematic review may be applied in interventional pain management practices utilizing appropriate evaluations (287). In this systematic review, mostly active-control trials or practical clinical trials were utilized. Practical clinical trials measure effectiveness. Consequently, these are considered more appropriate than explanatory trials meeting efficacy $(87,287,295-300)$. The differences between placebocontrol trials and active-control trials include the fact that placebo control trials measure absolute effect size and show the existence of the effect; whereas, activecontrol trials, not only show the existence of effect, but compared the therapies (256). Thus, the results of this systematic review may be considered generalizable if appropriate selection criteria are utilized.

The limitations of this study include that we were unable to perform meta-analysis for disc herniation, and the paucity of evidence for discogenic pain and spinal stenosis. Further, methodological criteria has been highly variable along with sample sizes. The studies were heterogenous. The results of this systematic review have significant implications for clinical practice. Interlaminar epidural injections show a significant reduction of pain scores in patients with lumbar radiculitis when compared to doing nothing, and conservative management without injection therapy.

\section{Acknowledgments}

The authors wish to thank Vidyasagar Pampati, MSc, for statistical assistance; Sekar Edem for assistance in the search of the literature; Laurie Swick for manuscript review; and Tonie M. Hatton and Diane E. Neihoff, transcriptionists, for their assistance in preparation of this manuscript. We would like to thank the editorial board of Pain Physician for review and criticism in improving the manuscript.

\section{References}

1. Manchikanti L, Boswell MV, Singh V, Benyamin RM, Fellows B, Abdi S, Buenaventura RM, Conn A, Datta S, Derby R, Falco FJE, Erhart S, Diwan S, Hayek SM, Helm S, Parr AT, Schultz DM, Smith HS, Wolfer LR, Hirsch JA. Comprehensive evidence-based guidelines for interventional techniques in the management of chronic spinal pain. Pain Physician 2009; 12:699-802.

2. Freburger JK, Holmes GM, Agans RP, Jackman AM, Darter JD, Wallace AS, Castel LD, Kalsbeek WD, Carey TS. The rising prevalence of chronic low back pain. Arch Intern Med 2009; 169:251-258.

3. Bressler HB, Keyes WJ, Rochon PA, Badley $E$. The prevalence of low back pain in the elderly. A systematic review of the literature. Spine (Phila Pa 1976) 1999; 24:1813-1819.

4. Ivanova JI, Birnbaum HG, Schiller $M$, Kantor E, Johnstone BM, Swindle RW. Real-world practice patterns, healthcare utilization, and costs in patients with low back pain: The long road to guideline-concordant care. Spine ] 2011; $11: 622-632$.
5.

Shiri R, Karppinen J, Leino-Arjas P, Solovieva S, Viikari-Juntura E. The association between obesity and low back pain: A meta-analysis. Am J Epidemiol 2010; 171:135-154.

6. Macfarlane $G$ J, Beasley $M$, Jones $E A$, Prescott GJ, Docking R, Keeley P, McBeth J, Jones GT; on behalf of the MUSICIAN study team. The prevalence and management of low back pain across adulthood: Results from a population-based crosssectional study (the MUSICIAN study). Pain 2012; 153:27-32.

7. Cassidy JD, Carroll LJ, Cotê P. The Saskatchewan Health and Back Pain Survey. The prevalence of low back pain and related disability in Saskatchewan Adults. Spine (Phila Pa 1976) 1998; 23:1860-1867.

8. Verhaak PF, Kerssens J, Dekker J, Sorbi MJ, Bensing JM. Prevalence of chronic benign pain disorder among adults: $A$ review of the literature. Pain 1998; 77:231239.

9. Luo X, Pietrobon R, Sun SX, Liu GG, Hey L. Estimates and patterns of direct health care expenditures among individuals with back pain in the United States.
Spine (Phila Pa 1976) 2004; 29:79-86.

10. Walker BF, Muller R, Grant WD. Low back pain in Australian adults: The economic burden. Asia Pac J Public Health 2003; 15:79-87.

11. Deyo RA, Mirza SK, Turner JA, Martin BI. Overtreating chronic back pain: Time to back off? J Am Board Fam Med 2009; 22:62-68.

12. Manchikanti L, Pampati V, Boswell MV, Smith HS, Hirsch JA. Analysis of the growth of epidural injections and costs in the Medicare population: A comparative evaluation of 1997, 2002, and 2006 data. Pain Physician 2010; 13:199-212.

13. Rubinstein SM, van Middelkoop M, Assendelft WJ, de Boer MR, van Tulder $M W$. Spinal manipulative therapy for chronic low-back pain: An update of a Cochrane review. Spine (Phila Pa 1976) 2011; 36:E825-E846.

14. Chou R, Qaseem A, Owens DK, Shekelle P; Clinical Guidelines Committee of the American College of Physicians. Diagnostic imaging for low back pain: Advice for high-value health care from the American College of Physicians. Ann In- 
tern Med 2011; 154:181-189.

15. Staal JB, de Bie RA, de Vet HC, Hildebrandt J, Nelemans P. Injection therapy for subacute and chronic low back pain: An updated Cochrane review. Spine (Phila Pa 1976) 2009; 34:49-59.

16. Kovacs FM, Urrútia G, Alarcón JD. Surgery versus conservative treatment for symptomatic lumbar spinal stenosis: A systematic review of randomized controlled trials. Spine (Phila Pa 1976) 2011; 36:E1335-E1351.

17. Jacobs WC, van Tulder M, Arts M, Rubinstein SM, van Middelkoop M, Ostelo $R$, Verhagen A, Koes B, Peul WC. Surgery versus conservative management of sciatica due to a lumbar herniated disc: A systematic review. Eur Spine J 2011; 20:513-522.

18. Baras JD, Baker LC. Magnetic resonance imaging and low back pain care for Medicare patients. Health Aff (Millwood) 2009; 28:w1133-w1140.

19. Chou R, Huffman L. Use of Chronic Opioid Therapy in Chronic Noncancer Pain: Evidence Review. American Pain Society, Glenview, IL, 2009.

www.ampainsoc.org/pub/pdf/Opioid_Final_ Evidence_Report.pdf

20. Chou R, Huffman L. Guideline for the Evaluation and Management of Low Back Pain: Evidence Review. American Pain Society, Glenview, IL, 2009.

www.ampainsoc.org/pub/pdf/LBPEvidRev.pdf

21. Manchikanti L, Fellows B, Ailinani H, Pampati V. Therapeutic use, abuse, and nonmedical use of opioids: A ten-year perspective. Pain Physician 2010; 13:401435 .

22. Manchikanti L, Ailinani $\mathrm{H}$, Koyyalagunta D, Datta S, Singh V, Eriator I, Sehgal N, Shah RV, Benyamin RM, Vallejo R, Fellows B, Christo PJ. A systematic review of randomized trials of long-term opioid management for chronic non-cancer pain. Pain Physician 2011; 14:91-121.

23. Manchikanti L, Vallejo R, Manchikanti KN, Benyamin RM, Datta S, Christo PJ. Effectiveness of long-term opioid therapy for chronic non-cancer pain. Pain Physician 2011; 14:E133-E156.

24. Manchikanti L, Pampati V, Singh V, Boswell MV, Smith HS, Hirsch JA. Explosive growth of facet joint interventions in the Medicare population in the United States: A comparative evaluation of 1997, 2002, and 2006 data. BMC Health Serv Res 2010; 10:84.

25. Manchikanti L, Singh V, Caraway DL, Benyamin RM, Hirsch JA. Medicare phy- sician payment systems: Impact of 2011 schedule on interventional pain management. Pain Physician 2011; 14: E5-E33.

26. Bogduk N, Christophidis N, Cherry D. Epidural use of steroids in the management of back pain. Report of working party on epidural use of steroids in the management of back pain. National Health and Medical Research Council, Canberra, Commonwealth of Australia, 1994, pp 1-76.

27. Manchikanti L, Datta S, Derby R, Wolfer LR, Benyamin RM, Hirsch JA. A critical review of the American Pain Society clinical practice guidelines for interventional techniques: Part 1. Diagnostic interventions. Pain Physician 2010; 13:E141E174.

28. Manchikanti L, Datta S, Gupta S, Munglani R, Bryce DA, Ward SP, Benyamin RM, Sharma ML, Helm II S, Fellows B, Hirsch JA. A critical review of the American Pain Society clinical practice guidelines for interventional techniques: Part 2. Therapeutic interventions. Pain Physician 2010; 13:E215-E264.

29. Manchikanti L, Singh V, Boswell MV. Interventional pain management at crossroads: The perfect storm brewing for a new decade of challenges. Pain Physician 2010; 13:E111-E140.

30. Chopko B, Caraway DL. MiDAS I (mild® Decompression Alternative to Open Surgery): A preliminary report of a prospective, multi-center clinical study. Pain Physician 2010; 13:369-378.

31. Manchikanti L, Helm II S, Hirsch JA. The evolution of the Patient-Centered Outcome Research Institute. ] Neurointervent Surg 2011; Published Online: August 31, 2011.

32. Manchikanti L, Hirsch JA. The Independent Payment Advisory Board: Impact on neurointerventionalists. J Neurointerv Surg 2011; Published Online: October 11, 2011.

33. Manchikanti L, Falco FJE, Boswell MV, Hirsch JA. Facts, fallacies, and politics of comparative effectiveness research: Part 1. Basic considerations. Pain Physician 2010; 13:E23-E54.

34. Manchikanti L, Falco FJE, Boswell MV, Hirsch JA. Facts, fallacies, and politics of comparative effectiveness research: Part 2. Implications for interventional pain management. Pain Physician 2010; 13:E55-E79.

35. Manchikanti L, Parr AT, Singh V, Fellows B. Ambulatory surgery centers and interventional techniques: A look at long-term survival. Pain Physician 2011; 14:E177-E215.

36. Manchikanti L, Falco FJ, Benyamin RM, Helm S 2nd, Parr AT, Hirsch JA. The impact of comparative effectiveness research on interventional pain management: Evolution from Medicare Modernization Act to Patient Protection and Affordable Care Act and the PatientCentered Outcomes Research Institute. Pain Physician 2011; 14:E249-E282.

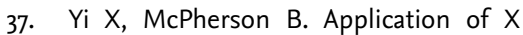
STOP device in the treatment of lumbar spinal stenosis. Pain Physician 2010; 13:E327-E336.

38. Benyamin RM, Datta S, Falco FJE. A perfect storm in interventional pain management: Regulated, but unbalanced. Pain Physician 2010; 13:109-116.

39. Manchikanti L, Hirsch JA. Medicare physician payment rules for 2011: A primer for the neurointerventionalist. AJNR Am J Neuroradiol 2011; 32:E101-E104.

40. Manchikanti L, Hirsch JA. Medicare physician payment rules for 2011: A primer for the neurointerventionalist. J Neurointervent Surg 2011; 3:399-402.

41. Deyo RA, Mirza SK, Martin BI, Kreuter W, Goodman DC, Jarvik JG. Trends, major medical complications, and charges associated with surgery for lumbar spinal stenosis in older adults. JAMA 2010; 303:1259-1265.

42. Chen $E$, Tong KB, Laouri M. Surgical treatment patterns among Medicare beneficiaries newly diagnosed with lumbar spinal stenosis. Spine ] 2010; 10:588594.

43. Cummins J, Lurie JD, Tosteson TD, Hanscom B, Abdu WA, Birkmeyer NJ, Herkowitz $H$, Weinstein J. Descriptive epidemiology and prior healthcare utilization of patients in the Spine Patient Outcomes Research Trial's (SPORT) three observational cohorts: Disc herniation, spinal stenosis, and degenerative spondylolisthesis. Spine (Phila Pa 1976) 2006; 31:806-814.

44. Haig AJ, Tomkins CC. Diagnosis and management of lumbar spinal stenosis. JAMA 2010; 303:71-72.

45. Tosteson AN, Tosteson TD, Lurie JD, Abdu W, Herkowitz H, Andersson G, Albert T, Bridwell K, Zhao W, Grove MR, Weinstein MC, Weinstein JN. Comparative effectiveness evidence from the spine patient outcomes research trial: Surgical versus nonoperative care for spinal stenosis, degenerative spondylolisthesis, and intervertebral disc hernia- 
tion. Spine (Phila Pa 1976) 2011; 36:20612068.

46. Parr AT, Diwan S, Abdi S. Lumbar interlaminar epidural injections in managing chronic low back and lower extremity pain: A systematic review. Pain Physician 2009; 12:163-188.

47. Conn A, Buenaventura R, Datta S, Abdi $S$, Diwan S. Systematic review of caudal epidural injections in the management of chronic low back pain. Pain Physician 2009; 12:109-135.

48. Buenaventura RM, Datta S, Abdi S Smith HS. Systematic review of therapeutic lumbar transforaminal epidural steroid injections. Pain Physician 2009; 12:233-251.

49. Manchikanti L, Singh V, Derby R, Schultz DM, Benyamin RM, Prager JP, Hirsch JA. Reassessment of evidence synthesis of occupational medicine practice guidelines for interventional pain management. Pain Physician 2008; 11:393482.

50. Bogduk N. Epidural steroids for low back pain and sciatica. Pain Digest 1999; 9:226-227.

51. Kepes ER, Duncalf D. Treatment of backache with spinal injections of local anesthetics, spinal and systemic steroids. Pain 1985; 22:33-47.

52. Koes BW, Scholten RJ, Mens JM, Bouter LM. Efficacy of epidural steroid injections for low back pain and sciatica: A systematic review of randomized clinical trials. Pain 1995; 63:279-288.

53. Koes BW, Scholten RJ, Mens JMA, Bouter LM. Epidural steroid injections for low back pain and sciatica: An updated systematic review of randomized clinical trials. Pain Digest 1999; 9:241-247.

54. Watts RW, Silagy CA. A meta-analysis on the efficacy of epidural corticosteroids in the treatment of sciatica. Anaesth Intensive Care 1995; 23:564-569.

55. Airaksinen O, Brox JI, Cedraschi C, Hildebrandt J, Klaber-Moffett J, Kovacs F, Mannion AF, Reis S, Staal JB, Ursin H, Zanoli G. Chapter 4: European guidelines for the management of chronic nonspecific low back pain. Eur Spine J 2006; 15:S192-S300.

56. Armon C, Argoff CE, Samuels J, Backonja MM; Therapeutics and Technology Assessment Subcommittee of the American Academy of Neurology. Assessment: Use of epidural steroid injections to treat radicular lumbosacral pain: Report of the Therapeutics and Technology Assessment Subcommittee of the Amer- ican Academy of Neurology. Neurology 2007; 68:723-729.

57. Denniston PL. Official Disability Guidelines. 13th ed. Work Loss Data Institute, 2008.

58. American College of Occupational and Environmental Medicine. Low Back Disorders Chapter. In: Occupational Medicine Practice Guidelines: Evaluation and Management of Common Health Problems and Functional Recovery of Workers. Second Edition. American College of Occupational and Environmental Medicine, Elk Grove Village, 2007.

59. Benzon HT. Epidural steroid injections for low back pain and lumbosacral radiculopathy. Pain 1986; 24:277-295.

6o. Jensen RK, Leboeuf-Yde C. Is the presence of Modic changes associated with the outcomes of different treatments? A systematic critical review. BMC Musculoskelet Disord 2011; 12:183

61. Weil L, Frauwirth $\mathrm{NH}$, Amirdelfan $\mathrm{K}$, Grant D, Rosenberg JA. Fluoroscopic analysis of lumbar epidural contrast spread after lumbar interlaminar injection. Arch Phys Med Rehabil 2008; 89:413-416.

62. Alemo S, Sayadipour A. Observational study of the use of an epidurogram in interlaminar lumbar epidural steroid injection. Br J Anaesth 2010; 104:665-666.

63. Cluff R, Mehio AK, Cohen SP, Chang Y, Sang CN, Stojanovic MP. The technical aspects of epidural steroid injections: A national survey. Anesth Analg 2002; 95:403-408.

64. Rathmell JP, Desjardins $A E$, van der Voort $M$, Hendriks $B H$, Nachabe $R$, Roggeveen $S$, Babic $D$, Söderman $M$, Brynolf M, Holmström B. Identification of the epidural space with optical spectroscopy: An in vivo swine study. Anesthesiology 2010; 113:1406-1418.

65. Choi YK, Barbella JD. Evaluation of epidurographic contrast patterns with fluoroscopic-guided lumbar interlaminar ventral epidural injection. Pain Pract 2009; 9:275-281.

66. White AH, Derby R, Wynne G. Epidural injections for the diagnosis and treatment of low-back pain. Spine (Phila Pa 1976) 1980; 5:78-86.

67. Fredman B, Nun MB, Zohar E, Iraqi G, Shapiro M, Gepstein R, Jedeikin R. Epidural steroids for treating "failed back surgery syndrome": Is fluoroscopy really necessary? Anesth Analg 1999; 88:367372.

68. Bartynski WS, Grahovac SZ, Rothfus
WE. Incorrect needle position during lumbar epidural steroid administration: Inaccuracy of loss of air pressure resistance and requirement of fluoroscopy and epidurography during needle insertion. AJNR Am J Neuroradiol 2005; 26:502-505.

69. Liu SS, Melmed AP, Klos JW, Innis CA. Prospective experience with a 20-gauge Tuohy needle for lumbar epidural steroid injections: Is confirmation with fluoroscopy necessary? Reg Anesth Pain Med 2001; 26:143-146.

70. Whitlock EL, Bridwell KH, Gilula LA. Influence of needle tip position on injectate spread in 406 interlaminar lumbar epidural steroid injections. Radiology 2007; 243:801-811.

71. Rabinovitch DL, Peliowski A, Furlan AD. Influence of lumbar epidural injection volume on pain relief for radicular leg pain and/or low back pain. Spine J 2009; 9:509-517.

72. Candido KD, Raghavendra MS, Chinthagada M, Badiee S, Trepashko DW. A prospective evaluation of iodinated contrast flow patterns with fluoroscopically guided lumbar epidural steroid injections: The lateral parasagittal interlaminar epidural approach versus the transforaminal epidural approach. Anesth Analg 2008; 106:638-644.

73. Botwin KP, Natalicchio J, Hanna A. Fluoroscopic guided lumbar interlaminar epidural injections: A prospective evaluation of epidurography contrast patterns and anatomical review of the epidural space. Pain Physician 2004; 7:7780.

74. Lenoir T, Deloin X, Dauzac C, Rillardon L, Guigui P. Paraplegia after interlaminar epidural steroid injection: A case report. Rev Chir Orthop Reparatrice Appar Mot 2008; 94:697-701.

75. Murthy NS, Maus TP, Behrns CL. Intraforaminal location of the great anterior radiculomedullary artery (artery of Adamkiewicz): A retrospective review. Pain Med 2010; 11:1756-1764.

76. Houten JK, Errico TJ. Paraplegia after lumbosacral nerve root block: Report of three cases. Spine ] 2002; 2:70-75.

77. Huntoon MA, Martin DP. Paralysis after transforaminal epidural injection and previous spinal surgery. Reg Anesth Pain Med 2004; 29:494-495.

78. Kennedy DJ, Dreyfuss P, Aprill CN, Bogduk N. Paraplegia following imageguided transforaminal lumbar spine epidural steroid injection: Two case re- 
ports. Pain Med 2009; 10:1389-1394.

79. Lyders EM, Morris PP. A case of spinal cord infarction following lumbar transforaminal epidural steroid injection: MR imaging and angiographic findings. Am J Neuroradiol 2009; 30:1691-1693.

8o. Somayaji HS, Saifuddin A, Casey AT, Briggs TW. Spinal cord infarction following therapeutic computed tomography-guided left L2 nerve root injection. Spine (Phila Pa 1976) 2005; 30:E106E108.

81. Wybier M, Gaudart S, Petrover D, Houdart E, Laredo JD. Paraplegia complicating selective steroid injections of the lumbar spine. Report of five cases and review of the literature. Eur Radiol 2010; 20:181-189.

82. Glaser SE, Shah RV. Root cause analysis of paraplegia following transforaminal epidural steroid injections: The "unsafe" triangle. Pain Physician 2010; 13:237-244.

83. Glaser SE, Falco F. Paraplegia following a thoracolumbar transforaminal epidural steroid injection. Pain Physician 2005; 8:309-314.

84. Scanlon GC, Moeller-Bertram T, Romanowsky SM, Wallace MS. Cervical transforaminal epidural steroid injections. More dangerous than we think? Spine (Phila Pa 1976) 2007; 32:1249-1256.

85. Rho ME, Tang CT. The efficacy of lumbar epidural steroid injections: Transforaminal, interlaminar, and caudal approaches. Phys Med Rehabil Clin N Am 2011; 22:139-148.

86. Manchikanti L, Hirsch JA, Smith HS. Evidence-based medicine, systematic reviews, and guidelines in interventional pain management: Part 2: Randomized controlled trials. Pain Physician 2008; 11:717-773.

87. Manchikanti L, Benyamin RM, Helm S, Hirsch JA. Evidence-based medicine, systematic reviews, and guidelines in interventional pain management: Part 3: Systematic reviews and meta-analysis of randomized trials. Pain Physician 2009; 12:35-72.

88. Moher D, Cook DJ, Eastwood S, Olkin I, Rennie D, Stroup DF. Improving the quality of reports of meta-analyses of randomised controlled trials: The QUOROM statement. Quality of reporting of meta-analyses. Lancet 1999; 354:18961900.

89. Liberati A, Altman DG, TetzlaffJ, Mulrow C, Gøtzsche PC, loannidis JP, Clarke M, Devereaux PJ, Kleijnen J, Moher D. The PRISMA statement for reporting sys- tematic reviews and meta-analyses of studies that evaluate health care interventions: Explanation and elaboration. Ann Intern Med 2009; 151:W65-W94.

90. van Tulder M, Furlan A, Bombardier C, Bouter L; Editorial Board of the Cochrane Collaboration Back Review Group. Updated method guidelines for systematic reviews in the Cochrane Collaboration Back Review Group. Spine (Phila Pa 1976) 2003; 28:1290-1299.

91. Furlan AD, Pennick V, Bombardier C, van Tulder M; Editorial Board, Cochrane Back Review Group. 2009 updated method guidelines for systematic reviews in the Cochrane Back Review Group. Spine (Phila Pa 1976) 2009; 34:1929-1941.

92. van Tulder MW, Suttorp $M$, Morton S Bouter LM, Shekelle P. Empirical evidence of an association between internal validity and effect size in randomized controlled trials of low-back pain. Spine (Phila Pa 1976) 2009; 34:1685-1692.

93. Manchikanti L, Singh V, Helm S, Schultz DM, Datta S, Hirsch J. An introduction to an evidence-based approach to interventional techniques in the management of chronic spinal pain. Pain Physician 2009; 12:E1-E33.

94. Stroup DF, Berlin JA, Morton SC, Olkin I, Williamson GD, Rennie D, Moher D, Becker BJ, Sipe TA, Thacker SB. Metaanalysis of observational studies in epidemiology: A proposal for reporting. Meta-analysis of Observational Studies in Epidemiology (MOOSE) group. JAMA 2000; 283:2008-2012.

95. Parr AT, Manchikanti L, Hameed $\mathrm{H}$, Conn A, Manchikanti KN, Benyamin RM, Diwan S, Singh V, Abdi S. Caudal epidural injections in the management of chronic low back pain: A systematic appraisal of the literature. Pain Physician 2012; 15:E159-E198.

96. Manchikanti L, Buenaventura RM, Manchikanti KN, Ruan X, Gupta S, Smith HS, Christo PJ, Ward SP. Effectiveness of therapeutic lumbar transforaminal epidural steroid injections in managing lumbar spinal pain. Pain Physician 2012; 15:E199-E245.

97. Altman DG, Schulz KF, Moher D, Egger M, Davidoff F, Elbourne D, Gøtzsche PC, Lang T; CONSORT GROUP (Consolidated Standards of Reporting Trials). The revised CONSORT statement for reporting randomized trials: Explanation and elaboration. Ann Intern Med 2001; 134:663-694.
98. Moher D, Hopewell S, Schulz KF, Montori V, Gøtzsche PC, Devereaux PJ, Elbourne D, Egger M, Altman DG. CONSORT 2010 explanation and elaboration: Updated guidelines for reporting parallel group randomised trials. BM] 2010; 340:c869.

99. Moher D, Schulz KF, Altman DG; CONSORT Group (Consolidated Standards of Reporting Trials). The CONSORT statement: Revised recommendations for improving the quality of reports of parallel-group randomized trials. J Am Podiatr Med Assoc 2001; 91:437-442.

100. Moher D, Schulz KF, Altman DG; CONSORT. The CONSORT statement: Revised recommendations for improving the quality of reports of parallel group randomized trials. BMC Med Res Methodol 2001; 1:2.

101. Vandenbroucke JP, von Elm E, Altman DG, Gøtzsche PC, Mulrow CD, Pocock S), Poole C, Schlesselman J, Egger M, STROBE Initiative. Strengthening the Reporting of Observational Studies in Epidemiology (STROBE): Explanation and elaboration. Ann Intern Med 2007; 147: W163-W194.

102. Staal JB, de Bie R, de Vet HC, Hildebrandt J, Nelemans P. Injection therapy for subacute and chronic low-back pain. Cochrane Database Syst Rev 2008; 3:CDool824.

103. Wells GA, Shea B, O'Connell D, Peterson J, Welch V, Losos M, Tugwell P. The Newcastle-Ottawa Scale (NOS) for assessing the quality of nonrandomized studies in meta-analysis. www.ohri.ca/ programs/clinical_epidemiology/oxford.asp

104. Higgins JP, Thompson SG, Deeks JJ, Altman DG. Measuring inconsistency in meta-analyses. BM] 2003; 327:557-560.

105. DerSimonian R, Laird N. Meta-analysis in clinical trials. Control Clin Trials 1986; 7:177-188.

106. Farrar JT. What is clinically meaningful: Outcome measures in pain clinical trials. Clin J Pain 2000; 16:S106-S112.

107. Salaffi F, Stancati A, Silvestri CA, Ciapetti A, Grassi W. Minimal clinically important changes in chronic musculoskeletal pain intensity measured on a numerical rating scale. Eur J Pain 2004; 8:283-291.

108. Bombardier C. Outcome assessments in the evaluation of treatment of spinal disorders: Summary and general recommendations. Spine (Phila Pa 1976) 2000; 25:3100-3103. 
109. Hagg O, Fritzell P, Nordwall A. The clinical importance of changes in outcome scores after treatment for chronic low back pain. Eur Spine J 2003; 12:12-20.

110. Manchikanti L, Cash KA, McManus CD, Pampati V, Benyamin RM. Preliminary results of a randomized, double-blind, controlled trial of fluoroscopic lumbar interlaminar epidural injections in managing chronic lumbar discogenic pain without disc herniation or radiculitis. Pain Physician 2010; 13:E279-E292.

111. Manchikanti L, Singh V, Falco FJE, Cash KA, Pampati V. Evaluation of the effectiveness of lumbar interlaminar epidural injections in managing chronic pain of lumbar disc herniation or radiculitis: A randomized, double-blind, controlled trial. Pain Physician 2010; 13:343-355.

112. Manchikanti L, Cash RA, McManus CD, Pampati V, Fellows B. Fluoroscopic caudal epidural injections with or without steroids in managing pain of lumbar spinal stenosis: One year results of randomized, double-blind, active-controlled trial. J Spinal Disord 2012; 25:226234.

113. Manchikanti L, Singh V, Cash KA, Pampati V, Damron KS, Boswell MV. A randomized, controlled, double-blind trial of fluoroscopic caudal epidural injections in the treatment of lumbar disc herniation and radiculitis. Spine (Phila Pa 1976) 2011; 36:1897-1905.

114. Manchikanti L, Singh V, Cash KA, Datta S. Management of pain of post lumbar surgery syndrome: One-year results of a randomized, double-blind, active controlled trial of fluoroscopic caudal epidural injections. Pain Physician 2010 13:509-521.

115. Manchikanti L, Singh V, Falco FJE, Cash KA, Pampati V. Evaluation of lumbar facet joint nerve blocks in managing chronic low back pain: A randomized, doubleblind, controlled trial with a 2-year follow-up. Int J Med Sci 2010; 7:124-135.

116. Manchikanti L, Singh V, Falco FJE, Cash KA, Fellows B. Comparative outcomes of a 2-year follow-up of cervical medial branch blocks in management of chronic neck pain: A randomized, double-blind controlled trial. Pain Physician 2010; 13:437-450.

117. Manchikanti L, Cash KA, Pampati V, Wargo BW, Malla Y. Cervical epidural injections in chronic discogenic neck pain without disc herniation or radiculitis: Preliminary results of a randomized, double-blind, controlled trial. Pain Phy- sician 2010; 13:E265-E278.

118. Manchikanti L, Cash KA, Pampati V Wargo BW, Malla Y. The effectiveness of fluoroscopic cervical interlaminar epidural injections in managing chronic cervical disc herniation and radiculitis: Preliminary results of a randomized, double-blind, controlled trial. Pain Physician 2010; 13:223-236.

119. Manchikanti L, Cash KA, McManus CD, Pampati V, Benyamin RM. A preliminary report of a randomized double-blind, active controlled trial of fluoroscopic thoracic interlaminar epidural injections in managing chronic thoracic pain. Pain Physician 2010; 13:E357-E369.

120. Manchikanti L, Singh V, Falco FJE, Cash KA, Pampati V, Fellows B. Comparative effectiveness of a one-year follow-up of thoracic medial branch blocks in management of chronic thoracic pain: A randomized, double-blind active controlled trial. Pain Physician 2010; 13:535-548.

121. Harbord R, Higgins J. METAREG: Stata module to perform meta-analysis regression. Boston College Department of Economics, Boston, MA. http://econpapers.repec.org/software/bocbocode/ S446201.htm

122. SPSS Statistics Software Standard Version (for Windows). Version 9.0.1. Chicago, IL, SPSS Inc; 1999.

123. Huedo-Medina TB, Sánchez-Meca J, Marín-Martínez F, Botella J. Assessing heterogeneity in meta-analysis: $Q$ statistic or 12 index? Psychol Methods 2006; 11:193-206.

124. Harris RP, Helfand M, Woolf SH, Lohr KN, Mulrow CD, Teutsch SM, Atkins D; Methods Work Group, Third US Preventive Services Task Force. Current methods of the US Preventive Services Task Force. Am J Prevent Med 2001; 20:21-35.

125. Serrao JM, Marks RL, Morley SJ, Goodchild CS. Intrathecal midazolam for the treatment of chronic mechanical low back pain: A controlled comparison with epidural steroid in a pilot study. Pain 1992; 48:5-12.

126. Rodriguez Hernandez R, Flores Lopez F. Assessment of pain intensity in patients with diabetic polyneuropathy treated with peridural $2 \%$ lidocaine methylprednisolone acetate vs peridural $2 \% \mathrm{li}$ docaine. Anestesia en Mexico 1999; 11:6569.

127. Kikuchi A, Kotani N, Sato T, Takamura K, Sakai I, Matsuki A. Comparative therapeutic evaluation of intrathecal versus epidural methylprednisolone for long- term analgesia in patients with intractable postherpetic neuralgia. Reg Anesth Pain Med 1999; 24:287-293.

128. Buchner M, Zeifang F, Brocai DR, Schiltenwolf M. Epidural corticosteroid injection in the conservative management of sciatica. Clin Orth Rel Res 2000; 375:149-156.

129. McGregor AH, Anjarwalla NK, Stambach T. Does the method of injection alter the outcome of epidural injections? ] Spinal Disord 2001; 14:507-510.

130. Dilke TF, Burry HC, Grahame R. Extradural corticosteroid injection in the management of lumbar nerve root compression. Br Med J 1973; 2:635-637.

131. Klenerman L, Greenwood R, Davenport HT, White DC, Peskett S. Lumbar epidural injections in the treatment of sciatica. BrJ Rheumatol 1984; 23:35-38.

132. Rocco AG, Frank E, Kaul AF, Lipson SJ, Gallo JP. Epidural steroids, epidural morphine and epidural steroids combined with morphine in the treatment of post-laminectomy syndrome. Pain 1989; 36:297-303.

133. Rogers $\mathrm{P}$, Nash T, Schiller D, Norman J. Epidural steroids for sciatica. Pain Clinic 1992; 5:67-72.

134. Valat JP, Giraudeau B, Rozenberg S, Goupille P, Bourgeois P, Micheau-Beaugendre V, Soubrier M, Richard S, Thomas E. Epidural corticosteroid injections for sciatica: A randomised, double blind, controlled clinical trial. Ann Rheum Dis 2003; 62:639-643.

135. Kraemer J, Ludwig J, Bickert U, Owczarek V, Traupe M. Lumbar epidural perineural injection: A new technique. Eur Spine J 1997; 6:357-361.

136. Pirbudak L, Karakurum G, Oner U, Gulec A, Karadasli H. Epidural corticosteroid injection and amitriptyline for the treatment of chronic low back pain associated with radiculopathy. Pain Clinic 2003; 15:247-253.

137. Bronfort G, Evans RL, Maiers M, Anderson AV. Spinal manipulation, epidural injections, and self-care for sciatica: A pilot study for a randomized clinical trial. J Manipulative Physiol Ther 2004; 278:503-508.

138. Briggs VG, Li W, Kaplan MS, Eskander MS, Franklin PD. Injection treatment and back pain associated with degenerative lumbar spinal stenosis in older adults. Pain Physician 2010 13:E347-E355.

139. Arden NK, Price C, Reading I, Stubbing J, Hazelgrove J, Dunne C, Michel M, Rogers P, Cooper C; WEST Study Group. 
A multicentre randomized controlled trial of epidural corticosteroid injections for sciatica: The WEST study. Rheumatology (Oxford) 2005; 44:1399-1406.

140. Carette S, Leclaire R, Marcoux S, Morin F, Blaise GA, St-Pierre A, Truchon R, Parent $F$, Levesque J, Bergeron $V$, Montminy $\mathrm{P}$, Blanchette $\mathrm{C}$. Epidural corticosteroid injections for sciatica due to herniated nucleus pulposus. $N$ Engl J Med 1997; 336:1634-1640.

141. Cuckler JM, Bernini PA, Wiesel SW, Booth RE Jr, Rothman RH, Pickens GT. The use of epidural steroid in the treatment of radicular pain. J Bone Joint Surg 1985; 67:63-66.

142. Wilson-MacDonald J, Burt G, Griffin D, Glynn C. Epidural steroid injection for nerve root compression: A randomized, controlled trial. J Bone Joint Surg $\mathrm{Br}$ 2005; 87-B:352-355.

143. Snoek W, Weber H, Jorgensen B. Double-blind evaluation of extradural methylprednisolone for herniated lumbar disc. Acta Orthop Scand 1977; 48:635-641.

144. Ridley MG, Kingsley GH, Gibson T, Grahame R. Outpatient lumbar epidural corticosteroid injection in the management of sciatica. Br J Rheumatol 1988 27:295-299.

145. Buttermann GR. The effect of spinal steroid injections for degenerative disc disease. Spine J 2004; 4:495-505.

146. Campbell MJ, Carreon LY, Glassman SD, McGinnis MD, Elmlinger BS. Correlation of spinal canal dimensions to efficacy of epidural steroid injection in spinal stenosis. J Spinal Disord Tech 2007; 20:168-171.

147. Manchikanti L, Cash KA, McManus CD, Damron KS, Pampati V, Falco FJE. Lumbar interlaminar epidural injections in central spinal stenosis: Preliminary results of a randomized double-blind control trial. Pain Physician 2012; 15:51-63.

148. Jirarattanaphochai $K$, Jung $S$, Thienthong S, Krisanaprakornkit W, Sumananont C. Peridural methylprednisolone and wound infiltration with bupivacaine for postoperative pain control after posterior lumbar spine surgery: A randomized double-blinded placebo-controlled trial. Spine (Phila Pa 1976) 2007; 32:609-616.

149. Schaufele MK, Hatch L, Jones W. Interlaminar versus transforaminal epidural injections for the treatment of symptomatic lumbar intervertebral disc herniations. Pain Physician 2006; 9:361-366.

150. Price CM, Rogers PD, Prosser AS, Arden NK. Comparison of the caudal and lum- bar approaches to the epidural space. Ann Rheum Dis 2000; 59:879-882.

151. Kapural L, Mekhail N, Bena J, McLain R, Tetzlaff J, Kapural M, Mekhail M, Polk S. Value of the magnetic resonance imaging in patients with painful lumbar spinal stenosis (LSS) undergoing lumbar epidural steroid injections. Clin ] Pain 2007; 23:571-575

152. Rivest C, Katz JN, Ferrante M, Jamison $\mathrm{RN}$. Effects of epidural steroid injection on pain due to lumbar spinal stenosis or herniated disks: A prospective study. Arthritis Care Res 1998; 11:291-297.

153. Fukusaki M, Kobayashi I, Hara T, Sumikawa K. Symptoms of spinal stenosis do not improve after epidural steroid injection. Clin J Pain 1998; 14:148-151.

154. Bush K, Cowan N, Katz DE, Gishen P. The natural history of sciatica associated with disc pathology. A prospective study with clinical and independent radiologic follow-up. Spine (Phila Pa 1976) 1992; 17:1205-1212.

155. Power RA, Taylor GJ, Fyfe IS. Lumbar epidural injection of steroid in acute prolapsed intervertebral discs. A prospective study. Spine (Phila Pa 1976) 1992; 17:453-455.

156. Berman AT, Garbarino JL Jr, Fisher SM, Bosacco SJ. The effects of epidural injection of local anesthetics and corticosteroids on patients with lumbosciatic pain. Clin Orthop Relat Res 1984; 188:144151.

157. Bowman SJ, Wedderburn L, Whaley A, Grahame R, Newman S. Outcome assessment after epidural corticosteroid injection for low back pain and sciatica. Spine (Phila Pa 1976) 1993; 18:1345-1350.

158. Hopwood MB, Abram SE. Factors associated with failure of lumbar epidural steroids. Reg Anesth 1993; 18:238-243.

159. Koning HM, Koning AJ, Bruinen TCM, Koster HG, Heybroek E. The period of pain relief following a successful epidural steroid injection for low-back pain. Pain Clin 2002; 13:331-338.

160. Nawani DP, Agrawal S, Asthana V. Single shot epidural injection for cervical and lumbosaccral radiculopathies: A preliminary study. Korean J Pain 2010; 23:254257.

161. Lotfinia I, Khallaghi E, Meshkini A, Shakeri M, Shima M, Safaeian A. Interaoperative use of epidural methylprednisolone or bupivacaine for postsurgical lumbar discectomy pain relief: A randomized, placebo-controlled trial. Ann Saudi Med 2007; 27:279-283.
162. Rasmussen S, Krum-Møller DS, Lauridsen LR, Jensen SE, Mandøe H, Gerlif C, Kehlet $\mathrm{H}$. Epidural steroid following discectomy for herniated lumbar disc reduces neurological impairment and enhances recovery: A randomized study with two-year follow-up. Spine (Phila Pa 1976) 2008; 33:2028-2033.

163. Mitra R, Huang L, Payne C. Epidural steroid injections in the management of a patient with spinal stenosis and urinary urgency. Nat Clin Pract Urol 2009; 6:113-115.

164. Stretanski MF. H-reflex latency and nerve root tension sign correlation in fluoroscopically guided, contrast-confirmed, translaminar lumbar epidural steroid-bupivacaine injections. Arch Phys Med Rehabil 2004; 85:1479-1482.

165. Lee JH, An JH, Lee SH. Comparison of the effectiveness of interlaminar and bilateral transforaminal epidural steroid injections in treatment of patients with lumbosacral disc herniation and spinal stenosis. Clin J Pain 2009; 25:206-210.

166. Nagda JV, Davis CW, Bajwa ZH, Simopoulos TT. Retrospective review of the efficacy and safety of repeated pulsed and continuous radiofrequency lesioning of the dorsal root ganglion/segmental nerve for lumbar radicular pain. Pain Physician 2011; 14:371-376.

167. Lima RM, Navarro LH, Carness JM, Barros GA, Marques ME, Solanki D, Ganem EM. Clinical and histological effects of the intrathecal administration of methylprednisolone in dogs. Pain Physician 2010; 13:493-501.

168. Park $\mathrm{CH}$, Lee $\mathrm{SH}$, Jung JY. Dural sac cross-sectional area does not correlate with efficacy of percutaneous adhesiolysis in single level lumbar spinal stenosis. Pain Physician 2011; 14:377-382.

169. Price C, Arden N, Coglan L, Rogers P. Cost-effectiveness and safety of epidural steroids in the management of sciatica. Health Technol Assess 2005; 9:1-58.

170. Debi R, Halperin N, Mirovsky Y. Local application of steroids following lumbar discectomy. J Spinal Disord Tech 2002; 15:273-276.

171. Aldrete JA. Epidural injections of indomethacin for postlaminectomy syndrome: A preliminary report. Anesth Analg 2003; 96:463-468.

172. Furman MB, Kothari G, Parikh T, Anderson JG, Khawaja A. Efficacy of fluoroscopically guided, contrast-enhanced lumbosacral interlaminar epidural steroid injections: A pilot study. Pain Med 
2010; 11:1328-1334.

173. Smith CC, Booker T, Schaufele MK Weiss P. Interlaminar versus transforaminal epidural steroid injections for the treatment of symptomatic lumbar spinal stenosis. Pain Med 2010; 11:15111515 .

174. Rados I, Sakic K, Fingler M, Kapural L. Efficacy of interlaminar vs transforaminal epidural steroid injection for the treatment of chronic unilateral radicular pain: Prospective, randomized study. Pain Med 2011; 12:1316-1321.

175. Noe CE, Haynsworth RF Jr. Comparison of epidural Depo-Medrol vs. aqueous betamethasone in patients with low back pain. Pain Pract 2003; 3:222-225.

176. Buttermann GR. Lumbar disc herniation regression after successful epidural steroid injection. J Spinal Disord Tech 2002; 15:469-476.

177. Buttermann GR. Treatment of lumbar disc herniation: Epidural steroid injection compared with discectomy. A prospective, randomized study. J Bone Joint Surg Am 2004; 86-A:670-679.

178. Lee JH, Moon J, Lee SH. Comparison of effectiveness according to different approaches of epidural steroid injection in lumbosacral herniated disk and spinal stenosis. ] Back Musculoskelet Rehabil 2009; 22:83-89.

179. Kim D, Brown J. Efficacy and safety of lumbar epidural dexamethasone versus methylprednisolone in the treatment of lumbar radiculopathy: A comparison of soluble versus particulate steroids. Clin J Pain 2011; 27:518-522.

180. Amr YM. Effect of addition of epidural ketamine to steroid in lumbar radiculitis: One-year follow-up. Pain Physician 2011; 14:475-481.

181. Ackerman WE 3rd, Ahmad M. The efficacy of lumbar epidural steroid injections in patients with lumbar disc herniations. Anesth Analg 2007; 104:1217-1222.

182. Lee JW, Shin HI, Park SY, Lee GY, Kang HS. Therapeutic trial of fluoroscopic interlaminar epidural steroid injection for axial low back pain: Effectiveness and outcome predictors. AJNR Am J Neuroradiol 2010; 31:1817-1823.

183. Gharibo CG, Varlotta GP, Rhame EE, Liu EC, Bendo JA, Perloff MD. Interlaminar versus transforaminal epidural steroids for the treatment of subacute lumbar radicular pain: A randomized, blinded, prospective outcome study. Pain Physician 2011; 14:499-511.

184. Gelalis ID, Arnaoutoglou E, Pakos EE,
Politis AN, Rapti M, Xenakis TA, Papadopoulos $\mathrm{G}$. Effect of interlaminar epidural steroid injection in acute and subacute pain due to lumbar disk herniation: A randomized comparison of 2 different protocols. Open Orthop J 2009; 3:121-124.

185. Heyse-Moore GH. A rational approach to the use of epidural medication in the treatment of sciatic pain. Acta Orthop Scand 1978; 49:366-370.

186. Jamison RN, VadeBoncouer T, Ferrante FM. Low back pain patients unresponsive to an epidural steroid injection: Identifying predictive factors. Clin J Pain 1991; 7:311-317.

187. Manchikanti L, Pakanati RR, Pampati V. Comparison of three routes of epidural steroid injections in low back pain. Pain Digest 1999; 9:277-285.

188. Evansa I, Vasilevskis E, Vanags I, Logina I, Borgeat A. Outcomes of lumbar epidural steroid injections - A cohort study. Periodicum Biologorum 2011; 113 Suppl 1:27.

189. Prochazka J, Hejcl A, Prochazkova L. Intrathecal midazolam as supplementary analgesia for chronic lumbar pain15 years' experience. Pain Med 2011; 12:1309-1315.

190. Sangheli M, Plesca S, Chetrari L. The role of epidural steroid injections in patients with multilevel lumbosacral herniated nucleus pulposus. Eur ] Pain Supple 2011; 5:290.

191. Dureja GP, Usmani H, Khan M, Tahseen $M$, Jamal A. Efficacy of intrathecal midazolam with or without epidural methylprednisolone for management of postherpetic neuralgia involving lumbosacral dermatomes. Pain Physician 2010; 13:213-221.

192. Möller JC, Cron RQ, Young DW, Girschick HJ, Levy DM, Sherry DD, Kukita A, Saijo K, Pessler F. Corticosteroidinduced spinal epidural lipomatosis in the pediatric age group: Report of a new case and updated analysis of the literature. Pediatr Rheumatol Online J 2011; 9:5.

193. Kapoor R, Liu J, Devasenapathy A, Gordin V. Gadolinium encephalopathy after intrathecal gadolinium injection. Pain Physician 2010; 13:E321-E326.

194. Koc Z, Ozcakir S, Sivrioglu K, Gurbet A, Kucukoglu S. Effectiveness of physical therapy and epidural steroid injections in lumbar spinal stenosis. Spine (Phila $\mathrm{Pa}$ 1976) 2009; 34:985-989.

195. Rosen CD, Kahanovitz N, Bernstein R, Viola K. A retrospective analysis of the efficacy of epidural steroid injections. Clin Orthop Relat Res 1988; 228:270-272.

196. Wang JC, Lin E, Brodke DS, Youssef JA. Epidural injections for the treatment of symptomatic lumbar herniated discs. J Spinal Disord Tech 2002; 15:269-272.

197. Mobaleghi J, Allahdini F, Nasseri K, Ahsan B, Shami S, Faizi M, Gharibi F. Comparing the effects of epidural methylprednisolone acetate injected in patients with pain due to lumbar spinal stenosis or herniated disks: A prospective study. Int J Gen Med 2011; 4:875-878.

198. Manchikanti L, Cash KA, McManus CD, Pampati V, Benyamin RM. Fluoroscopic lumbar interlaminar epidural injections in managing chronic lumbar axial or discogenic pain. J Pain Research 2012; in press.

199. Manchikanti L, Singh V, Cash KA, Pampati $\mathrm{V}$, Falco FJE. The role of fluoroscopic interlaminar epidural injections in managing chronic pain of lumbar disc herniation or radiculitis: A randomized, double-blind trial. Pain Pract 2012 Dec. 27. [Epub ahead of print].

200. Gutknecht DR. Chemical meningitis following epidural injections of corticosteroids (Letter). Am J Med 1987; 82:570.

201. Yue WM, Tan SB. Distant skip level discitis and vertebral osteomyelitis after caudal epidural injection: A case report of a rare complication of epidural injections. Spine (Phila Pa 1976) 2003; 28:E209-E211.

202. Kabbara A, Rosenberg SK, Untal C. Methicillin-resistant staphylococcus aureus epidural abscess after transforaminal epidural steroid injection. Pain Physician 2004; 7:269-272.

203. Hooten WM, Kinney MO, Huntoon MA. Epidural abscess and meningitis after epidural corticosteroid injection. Mayo Clin Proc 2004; 79:682-686.

204. Manchikanti L, Malla Y, Wargo BW, Fellows B. Infection control practices (safe injection and medication vial utilization) for interventional techniques: Are they based on relative risk management or evidence? Pain Physician 2011; 14:425434.

205. Simopoulos TT, Kraemer JJ, Glazer P, Bajwa ZH. Vertebral osteomyelitis: A potentially catastrophic outcome after lumbar epidural steroid injection. Pain Physician 2008; 11:693-697.

206. McGrath JM, Schaefer MP, Malkamaki DM. Incidence and characteristics of complications from epidural steroid injections. Pain Med 2011; 12:726-731.

207. Coumans JV, Walcott BP. Rapidly pro- 
gressive lumbar subdural empyema following acromial bursal injection. J Clin Neurosci 2011; 18:1562-1563.

208. Saigal G, Donovan Post MJ, Kozic D. Thoracic intradural Aspergillus abscess formation following epidural steroid injection. AJNR Am J Neuroradiol 2004; 25:642-644.

209. Torres-Ramos FM, Botwin K, Shah CP. Candida spondylodiscitis: An unusual case of thoracolumbar pain with review of imaging findings and description of the clinical condition. Pain Physician 2004; 7:257-260.

210. Ozdemir O, Calisaneller T, Yildirim E, Altinors N. Acute intracranial subdural hematoma after epidural steroid injection: A case report. J Manipulative Physiol Ther 2007; 30:536-538.

211. Yoo HS, Park SW, Han JH, Chung JY, Yi JW, Kang JM, Lee BJ, Kim DO. Paraplegia caused by an epidural hematoma in a patient with unrecognized chronic idiopathic thrombocytopenic purpura following an epidural steroid injection. Spine (Phila Pa 1976) 2009; 34: E376-E379.

212. Xu R, Bydon M, Gokaslan ZL, Wolinsky $J P$, Witham TF, Bydon A. Epidural steroid injection resulting in epidural hematoma in a patient despite strict adherence to anticoagulation guidelines. J Neurosurg Spine 2009; 11:358-364.

213. Shanthanna H, Park J. Acute epidural haematoma following epidural steroid injection in a patient with spinal stenosis. Anaesthesia 2011; 66:837-839.

214. LaBan MM, Kasturi G, Wang IM. Epidural corticosteroid injections precipitating epidural hematomas with spinal paresis. Am J Phys Med Rehabil 2007; 86:166-167.

215. Ain RJ, Vance MB. Epidural hematoma after epidural steroid injection in a patient withholding enoxaparin per guidelines. Anesthesiology 2005; 102:701-703.

216. Manchikanti L, Malla Y, Wargo BW, Cash KA, McManus CD, Damron KS, Jackson SD, Pampati V, Fellows B. A prospective evaluation of bleeding risk of interventional techniques in chronic pain. Pain Physician 2011; 14:317-329.

217. Nelson DA, Landau WM. Intraspinal steroids: History, efficacy, accidentality, and controversy with review of United States Food and Drug Administration reports. J Neurol Neurosurg Psychiatry 2001; 70:433-443.

218. Manchikanti L, Singh V. Corticosteroids. In: Manchikanti L, Christo PJ, Trescot
AM, Falco FJE (eds). Foundations of Pain Medicine and Interventional Pain Management: A Comprehensive Review. ASIPP Publishing, Paducah, KY, 2011, pp 589606.

219. Delaney TJ, Rowlingson JC, Carron $\mathrm{H}$, Butler A. Epidural steroid effects on nerves and meninges. Anesth Analg 1980; 58:610-614.

220. Abram SE, Marsala M, Yaksh TL. Analgesic and neurotoxic effects of intrathecal corticosteroids in rats. Anesthesiology 1994; 81:1198-1205.

221. Chino N, Awad EA, Kottke FJ. Pathology of propylene glycol administered by perineural and intramuscular injection in rats. Arch Phys Med Rehab 1974; 55:3338.

222. Latham JM, Fraser RD, Moore RJ, Blumbergs PC, Bogduk N. The pathologic effects of intrathecal betamethasone. Spine (Phila Pa 1976) 1997; 22:1558-1562.

223. Linn AJ, Desilva C, Peeters-Asdourian C. Thromboembolic stroke: A rare complication associated with peri-procedural management of an epidural steroid injection. Pain Physician 2009; 12:159-162.

224. Usta B, Muslu B. Sedation during cervical vs. lumbar translaminar epidural steroid injections. Pain Pract 2009; 9:480; author reply 480-481.

225. Thefenne L, Dubecq C, Zing E, Rogez D, Soula M, Escobar E, Defuentes G, Lapeyre $E$, Berets $O$. A rare case of paraplegia complicating a lumbar epidural infiltration. Ann Phys Rehabil Med 2010; 53:575-583.

226. Benzon HT, lqbal $M$, Tallman $M S$, Boehlke L, Russell EJ. Superior sagittal sinus thrombosis in a patient with postdural puncture headache. Reg Anesth Pain Med 2003; 28:64-67.

227. Hetts SW, Narvid J, Singh T, Meagher S, Corcoran K, Higashida RT, Dowd CF, Halbach VV. Association between lumbar epidural injection and development of acute paraparesis in patients with spinal dural arteriovenous fistulas. AJNR Am J Neuroradiol 2007; 28:581-583.

228. Schultz TE. Inadvertent discogram during epidural steroid injection: A case report. AANA J 2008; 76:189-191.

229. Candido KD, Katz JA, Chinthagada M, McCarthy RA, Knezevic NN. Incidence of intradiscal injection during lumbar fluoroscopically guided transforaminal and interlaminar epidural steroid injections. Anesth Analg 2010; 110:1464-1467.

230. Ammirati M, Perino F. Symptomatic air trapped in the spine after lumbar epidural corticosteroid injection. Case report. J Neurosurg Spine 2006; 5:359-361.

231. Lehmann LJ, Pallares VS. Subdural injection of a local anesthetic with steroids: Complication of epidural anesthesia. South Med J 1995; 88:467-469.

232. Purdy EP, Ajimal GS. Vision loss after lumbar epidural steroid injection. Anesth Analg 1998; 86:119-122.

233. Huang J, Kwa A. Lumbar discogram resulting from lumbar interlaminar epidural injection. J Clin Anesth 2004; 16:296-298.

234. Young WF. Transient blindness after lumbar epidural steroid injection: A case report and literature review. Spine (Phila Pa 1976) 2002; 27:E476-E477.

235. Browning DJ. Acute retinal necrosis following epidural steroid injections. Am J Ophthalmol 2003; 136:192-194.

236. Kusher $\mathrm{FH}$, Olson JC. Retinal hemorrhage as a consequence of epidural steroid injection. Arch Opthalmol 1995; 113:309-313.

237. Iida T, Spaide RF, Negrao SG, Carvalho CA, Yannuzzi LA. Central serous chorioretinopathy after epidural corticosteroid injection. Am J Ophthalmol 2001; 132:423-425.

238. Pizzimenti JJ, Daniel KP. Central serous chorioretinopathy after epidural steroids. Pharmacotherapy 2005; 25:11411146.

239. McAllister RK, McDavid AJ, Meyer TA, Bittenbinder TM. Recurrent persistent hiccups after epidural steroid injection and analgesia with bupivacaine. Anesth Analg 2005; 100:1834-1836.

240. Everett CR, Baskin MN, Novoseletsky D, Speach D, Patel R. Flushing as a side effect following lumbar transforaminal epidural steroid injection. Pain Physician 2004; 7:427-429.

241. Kim CH, Issa MA, Vaglienti RM. Flushing following interlaminar lumbar epidural steroid injection with dexamethasone. Pain Physician 2010; 13:481-484.

242. Somanchi BV, Mohammad S, Ross R. An unusual complication following caudal epidural steroid injection: A case report. Acta Orthop Belg 2008; 74:720-722.

243. Manchikanti L, Malla Y, Wargo BW, Fellows B. Preoperative fasting before interventional techniques: Is it necessary or evidence-based? Pain Physician 2011; 14:459-467.

244. Mosaffa F, Karimi K, Madadi F, Khoshn- 
evis SH, Besheli LD, Eajazi A. Post-dural puncture headache: $A$ comparison between median and paramedian approaches in orthopedic patients. Anesth Pain 2011; 1:66-69.

245. Gonzalez P, Laker SR, Sullivan W, Harwood JE, Akuthota V. The effects of epidural betamethasone on blood glucose in patients with diabetes mellitus. PM $R$ 2009; 1:340-345.

246. Gitkind Al, Shah B, Thomas M. Epidural corticosteroid injections as a possible cause of menorrhagia: A case report. Pain Med 2010; 11:713-715.

247. Hughes JM, Hichens M, Booze GW, Thorner MO. Cushing's syndromes from therapeutic use of intramuscular dexamethasone acetate. Arch Intern Med 1986; 146:1848-1849.

248. Maillefert JF, Aho S, Piroth-Chatard C, Tavernier C. Cortisol levels after single local steroid injection. Am J Med 1996; 100:586-587.

249. Boonen S, Van Distel G, Westhovens R, Dequeker J. Steroid myopathy induced by epidural triamcinolone injection. Brit J Rheumatol 1995; 34:385.

250. McCullen GM, Spurling GR, Webster JS Epidural lipomatosis complicating lumbar steroid injections. J Spinal Disord 1999; 12:526-529.

251. Manchikanti L, Malla Y, Wargo BW, Cash KA, Pampati V, Fellows B. A prospective evaluation of complications of 10,000 fluoroscopically directed epidural injections. Pain Physician 2012; 15:131-140.

252. Manchikanti L, Cash KA, Moss TL, Pampati V. Effectiveness of protective measures in reducing risk of radiation exposure in interventional pain management: A prospective evaluation. Pain Physician 2003; 6:301-305.

253. Manchikanti L, Cash KA, Moss TL, Rivera JJ, Pampati V. Risk of whole body radiation exposure and protective measures in fluoroscopically guided interventional techniques: A prospective evaluation. BMC Anesthesiol 2003; 3:2.

254. Manchikanti L, Cash KA, Moss TL, Pampati V. Radiation exposure to the physician in interventional pain management. Pain Physician 2002; 5:385-393.

255. American Society of Anesthesiologists Task Force on Chronic Pain Management; American Society of Regional Anesthesia and Pain Medicine. Practice guidelines for chronic pain management: An updated report by the American Society of Anesthesiologists Task
Force on Chronic Pain Management and the American Society of Regional Anesthesia and Pain Medicine. Anesthesiology 2010; 112:810-833.

256. Mehta M, Salmon N. Extradural block. Confirmation of the injection site by X-ray monitoring. Anaesthesia 1985; 40:1009-1012.

257. Burn JM, Guyer PB, Langdon L. The spread of solutions injected into the epidural space: A study using epidurograms in patients with lumbosciatic syndrome. Br J Anaesth 1973; 45:338-345.

258. Manchikanti L, Bakhit CE, Pakanati RR, Fellows B. Fluoroscopy is medically necessary for the performance of epidural steroids. Anesth Analg 1999; 89:13301331

259. Nishimura N, Khahara T, Kusakabe $\mathrm{T}$. The spread of lidocaine and 1-131 solution in the epidural space. Anesthesiology 1959; 20:785-788.

260. Manchikanti L, Dunbar EE. Correlation of spinal canal dimensions to efficacy of epidural steroid injection in spinal stenosis. J Spinal Disord Tech 2007; 20:546547.

261. Peterson C, Hodler J. Evidence-based radiology (part 1 ): Is there sufficient research to support the use of therapeutic injections for the spine and sacroiliac joints? Skeletal Radiol 2010; 39:5-9.

262. Carette $S$, Marcoux S, Truchon R, Grondin C, Gagnon J, Allard Y, Latulippe M. A controlled trial of corticosteroid injections into facet joints for chronic low back pain. N Engl J Med 1991; 325:10021007.

263. Karppinen J, Malmivaara A, Kurunlahti $M$, Kyllönen E, Pienimäki T, Nieminen P, Ohinmaa A, Tervonen O, Vanharanta $H$. Periradicular infiltration for sciatica: A randomized controlled trial. Spine (Phila Pa 1976) 2001; 26:1059-1067.

264. Manchikanti L, Giordano J, Fellows B, Hirsch JA. Placebo and nocebo in interventional pain management: A friend or a foe - or simply foes? Pain Physician 2011; 14:E157-E175.

265. Smuck M, Levin JH. RE: Manchikanti L, Singh V, Falco FJE, Cash KA, Fellows B. Cervical medial branch blocks for chronic cervical facet joint pain: A randomized double-blind, controlled trial with one-year follow-up. Spine (Phila Pa 1976) 2008; 33:1813-1820. Spine (Phila Pa 1976) 2009; 34:1116-1117.

266. Levin JH. Prospective, double-blind, randomized placebo-controlled trials in interventional spine: What the highest quality literature tells us. Spine J 2009; 9:690-703.

267. Manchikanti L, Shah RV, Datta S, Singh V. Critical evaluation of interventional pain management literature provides inaccurate conclusions. Spine J 2009; 9:706-708.

268. Nelemans PJ, Debie RA, DeVet HC, Sturmans F. Injection therapy for subacute and chronic benign low back pain. Spine (Phila Pa 1976) 2001; 26:501-515.

269. Pham Dang C, Lelong A, Guilley J, Nguyen JM, Volteau C, Venet G, Perrier $C$, Lejus C, Blanloeil Y. Effect on neurostimulation of injectates used for perineural space expansion before placement of a stimulating catheter: Normal saline versus dextrose $5 \%$ in water. Reg Anesth Pain Med 2009; 34:398-403.

270. Tsui BC, Kropelin B, Ganapathy S, Finucane B. Dextrose $5 \%$ in water: Fluid medium maintaining electrical stimulation of peripheral nerve during stimulating catheter placement. Acta Anaesthesiol Scand 2005; 49:1562-1565.

271. Indahl A, Kaigle AM, Reikeräs O, Holm $\mathrm{SH}$. Interaction between the porcine lumbar intervertebral disc, zygapophysial joints, and paraspinal muscles. Spine (Phila Pa 1976) 1997; 22:2834-2840.

272. Indahl A, Kaigle A, Reikeräs O, Holm S. Electromyographic response of the porcine multifidus musculature after nerve stimulation. Spine (Phila Pa 1976) 1995; 20:2652-2658.

273. Bhatia MT, Parikh LC). Epidural saline therapy in lumbo-sciatic syndrome. J Indian Med Assoc 1966; 47:537-542.

274. Gupta AK, Mital VK, Azmi RU. Observations of the management of lumbosciatic syndromes (sciatica) by epidural saline. J Indian Med Assoc 1970; 54:194-196.

275. Pasqualucci A, Varrassi G, Braschi A, Peduto VA, Brunelli A, Marinangeli $F$, Gori F, Colò F, Paladini A, Mojoli F. Epidural local anesthetic plus corticosteroid for the treatment of cervical brachial radicular pain: Single injection versus continuous infusion. Clin J Pain 2007; 23:551-557.

276. Byrod G, Otani K, Brisby H, Rydevik B, Olmarker K. Methylprednisolone reduces the early vascular permeability increase in spinal nerve roots induced by epidural nucleus pulposus application. J Orthop Res 2000; 18:983-987.

277. Hayashi N, Weinstein JN, Meller ST, Lee HM, Spratt KF, Gebhart GF. The effect of epidural injection of betamethasone or bupivacaine in a rat model of lumbar 
radiculopathy. Spine (Phila Pa 1976) 1998; 23:877-885.

278. Lee HM, Weinstein JN, Meller ST, Hayashi N, Spratt KF, Gebhart GF. The role of steroids and their effects on phospholipase A2: An animal model of radiculopathy. Spine (Phila Pa 1976) 1998; 23:1191-1196.

279. Minamide A, Tamaki T, Hashizume $\mathrm{H}$, Yoshida M, Kawakami M, Hayashi N. Effects of steroids and lipopolysaccharide on spontaneous resorption of herniated intervertebral discs: An experimental study in the rabbit. Spine (Phila Pa 1976) 1998; 23:870-876.

280. Mao J, Chen LL. Systemic lidocaine for neuropathic pain relief. Pain 2000; 87:717.

281. Pasqualucci A. Experimental and clinical studies about the preemptive analgesia with local anesthetics. Possible reasons of the failure. Minerva Anestesiol 1998; 64:445-457.

282. Arner S, Lindblom U, Meyerson BA, Molander C. Prolonged relief of neuralgia after regional anesthetic block. A call for further experimental and systematic clinical studies. Pain 1990; 43:287-297.

283. Lavoie PA, Khazen T, Filion PR. Mechanisms of the inhibition of fast axonal transport by local anesthetics. Neuropharmacology 1989; 28:175-181.

284. Ji RR, Woolf CJ. Neuronal plasticity and signal transduction in nociceptive neurons: Implications for the initiation and maintenance of pathological pain. Neurobiol Dis 2001; 8:1-10.

285. Cassuto J, Sinclair R, Bonderovic M. Anti-inflammatory properties of local anesthetics and their present and potential clinical implications. Acta Anaesthesiol Scand 2006; 50:265-282.

286. Manchikanti L, Cash KA, McManus CD, Pampati V, Singh V, Benyamin RM. The preliminary results of a comparative effectiveness evaluation of adhesiolysis and caudal epidural injections in managing chronic low back pain secondary to spinal stenosis: A randomized, equivalence controlled trial. Pain Physician 2009; 12:E341-E354.

287. Manchikanti L, Singh V, Cash KA, Pampati $\vee$, Datta S. A comparative effectiveness evaluation of percutaneous adhesiolysis and epidural steroid injections in managing lumbar post surgery syndrome: A randomized, equivalence controlled trial. Pain Physician 2009; 12: $\mathrm{E}_{355}-\mathrm{E}_{3} 68$.

288. Manchikanti L, Cash KA, McManus CD, Pampati V, Abdi S. Preliminary results of randomized, equivalence trial of fluoroscopic caudal epidural injections in managing chronic low back pain: Part 4. Spinal stenosis. Pain Physician 2008; 11:833-848.

289. Manchikanti L, Singh V, Cash KA, Pampati V, Damron KS, Boswell MV. Preliminary results of randomized, equivalence trial of fluoroscopic caudal epidural injections in managing chronic low back pain: Part 2. Disc herniation and radiculitis. Pain Physician 2008; 11:801-815.

290. Manchikanti L, Malla Y, Cash KA, McManus CD, Pampati V. Fluoroscopic cervical interlaminar epidural injections in managing chronic pain of cervical postsurgery syndrome: Preliminary results of a randomized, double-blind active control trial. Pain Physician 2012; 15:1326.

291. Manchikanti L, Malla Y, Cash KA, McManus CD, Pampati V. Fluoroscopic epidural injections in cervical spinal stenosis: Preliminary results of a randomized, double-blind, active control trial. Pain Physician 2012; 15:E59-E70.
292. Manchikanti L, Singh V, Falco FJ, Cash KA, Fellows B. Cervical medial branch blocks for chronic cervical facet joint pain: A randomized double-blind, controlled trial with one-year follow-up. Spine (Phila Pa 1976) 2008; 33:1813-1820.

293. Sato C, Sakai A, Ikeda Y, Suzuki H, Sakamoto $A$. The prolonged analgesic effect of epidural ropivacaine in a rat model of neuropathic pain. Anesth Analg 2008; 106:313-320.

294. Tachihara H, Sekiguchi M, Kikuchi S, Konno S. Do corticosteroids produce additional benefit in nerve root infiltration for lumbar disc herniation. Spine (Phila Pa 1976) 2008; 33:743-747.

295. Manchikanti L, Datta S, Smith HS, Hirsch JA. Evidence-based medicine, systematic reviews, and guidelines in interventional pain management: Part 6. Systematic reviews and meta-analyses of observational studies. Pain Physician 2009; 12:819-850.

296. Hotopf M. The pragmatic randomized controlled trial. Adv Psychiatr Treat 2002; 8:326-333.

297. Hotopf M, Churchill R, Lewis G. Pragmatic randomized controlled trials in psychiatry. Br J Psychiatry 1999; 175:217223.

298. Tunis SR, Stryer DB, Clancy CM. Practical clinical trials. Increasing the value of clinical research for decision making in clinical and health policy. JAMA 2003; 290:1624-1632.

299. Roland $M$, Torgerson DJ. What are pragmatic trials? $B M]$ 1998; 316:285.

300. Alexander GC, Stafford RS. Does comparative effectiveness have a comparative edge? JAMA 2009; 301:2488-2490. 